\title{
Strategies of molecular imprinting-based solid-phase extraction prior to chromatographic analysis
}

\author{
Maryam Arabi a , Abbas Ostovan ${ }^{\mathrm{a},{ }^{* *}}$, Ahmad Reza Bagheri ${ }^{\mathrm{b}}$, Xiaotong Guo ${ }^{\mathrm{a}}$, Liyan Wang ${ }^{\mathrm{a}}$, \\ Jinhua Li ${ }^{\text {a }}{ }^{\mathrm{f}}$, Xiaoyan Wang a, c, Bowei Li ${ }^{\mathrm{a}}$, Lingxin Chen ${ }^{\mathrm{a}, \mathrm{d}}$, e, * \\ ${ }^{a}$ CAS Key Laboratory of Coastal Environmental Processes and Ecological Remediation, Shandong Key Laboratory of Coastal Environmental Processes, \\ Research Center for Coastal Environmental Engineering and Technology, Yantai Institute of Coastal Zone Research, Chinese Academy of Sciences, Yantai \\ 264003, China \\ b Chemistry Department, Yasouj University, Yasouj 75918-74831, Iran \\ c School of Pharmacy, Binzhou Medical University, Yantai 264003, China \\ ${ }^{\mathrm{d}}$ Department of Chemistry and Chemical Engineering, Qufu Normal University, Qufu 273165, China \\ e School of Chemistry and Chemical Engineering, Yantai University, Yantai 264005, China \\ ${ }^{\mathrm{f}}$ Center for Ocean Mega-Science, Chinese Academy of Sciences, Qingdao 266071, China
}

\section{A R T I C L E I N F O}

Article history:

Available online 22 May 2020

\section{Keywords:}

Sample preparation

Molecularly imprinted polymers (MIPs)

Imprinting strategy

Solid-phase extraction (SPE)

Chromatographic analysis

\begin{abstract}
A B S T R A C T
Molecular imprinting-based solid-phase extraction (MI-SPE) has been in the spotlight to improve the recognition selectivity and detection sensitivity. MI-SPE provides a powerful tool for chemo/bioanalysis in complex matrices and meanwhile, benefits from distinguished advantages such as easy operation, high throughput, low cost, high selectivity and durability. This review proposed the recent advances in molecular imprinting concerning novel preparation strategies of molecularly imprinted polymers (MIPs) and typical applications of MI-SPE. Preparation strategies are highlighted by dividing into ten sections mainly including dummy imprinting, multi-template imprinting, surface imprinting, water-compatible imprinting, restricted access material combining imprinting etc.; each section provides the descriptions about what restrictions led to the emergence of any strategy, strengths/weaknesses of every strategy and universal applications of upgraded MIPs in various SPE modes prior to chromatographic analysis. The potential of MIPs for implementation in routine laboratory activities and scale-up is expected, and finally remaining challenges and future perspectives are proposed.
\end{abstract}

() 2020 Elsevier B.V. All rights reserved.

\section{Introduction}

Nowadays, analytical chemistry has played a fundamental role in qualitative/quantitative supervision over all aspects of our lives including industrial, pharmaceutical, food, clinical, cosmetic and environmental analysis [1,2]. Typically, qualitative/quantitative analysis of chemicals consists of three main steps, including (i) sampling, (ii) sample preparation, and (iii) instrumental

\footnotetext{
* Corresponding author. CAS Key Laboratory of Coastal Environmental Processes and Ecological Remediation, Shandong Key Laboratory of Coastal Environmental Processes, Research Center for Coastal Environmental Engineering and Technology, Yantai Institute of Coastal Zone Research, Chinese Academy of Sciences, Yantai 264003, China. Fax: +86 5352109130.

** Corresponding author.

E-mail addresses: saman.ostovan@yahoo.com (A. Ostovan), Ixchen@yic.ac.cn (L. Chen).
}

measurement. Since, most of the samples are heterogeneous, to guarantee the representativeness of throughout the bulk sample, a gross sample is generally thoroughly mixed and homogenized to obtain "laboratory sample", and then a small portion called "aliquot" is chosen for the further step. Often, samples with a rich matrix composition are not compatible with analytical instruments. Notwithstanding the vast development in analytical instrumentation and emerging of advanced hyphenated techniques, direct analysis of crude samples is an unattainable dream up to now for analytical chemists. Hence, sample pretreatment/ preparation has been impressively progressed to overcome the obstacle of the devices. For chromatography based techniques, the most popular and applicable sample preparation is extraction approaches [3]. Commonly, sample pretreatment has been performed by manipulating or instrumentation methods with the aim of isolation and preconcentration of the analyte of interest, elimination of matrix contaminants disturbing analyte detection and/or 


\section{List of abbreviations and acronyms}

2,4,6-TCP 2,4,6-trichlorophenol

2,4-DCP 2,4-dichlorophenol

2,6-DCP 2,6-dichlorophenol

2-CP 2-chlorophenol

4-CP 4-chlorophenol

4-VP 4-vinylpyridine

AA Acrylamide

AMPS 2-acrylamido-2-methylpropanesulfonic acid

APBA Aminophenylboronic acid

APGC-MS/MS Gas chromatography with an atmospheric pressure chemical ionization tandem mass spectrometry

APTMS 3-aminopropyltrimethoxysilane

BPA Bisphenol A

CBZ Carbendazim

CCT Cellulose cone tip

COF@MOF Covalent organic framework-metal organic framework

CS Carbon spheres

DINP Diisononyl phthalate

DLLME Dispersive liquid liquid microextraction

DMIPs Dummy molecularly imprinted polymers

DMMIPs Dummy magnetic molecularly imprinted nanoparticles

DMSO Dimethyl sulfoxide

DPRP Dipropyl phthalate

d-SPE Dispersive solid-phase extraction

EF Enrichment factor

EGDMA Ethylene glycol dimethacrylate

EMRL Enhanced matrix removal lipid

ESI-MS Electrospray ionization mass spectrometry

$\mathrm{Fe}_{3} \mathrm{O}_{4} @ G \mathrm{G}-\mathrm{DES}$ Magnetic graphene oxide-deep eutectic solvent

GC-ECD GC with an electron capture detector

GC-MS Gas chromatography-mass spectrometery

GMA Glycidyl methacrylate

GPC Gel permeation chromatography

HMIPs Hollow porous molecularly imprinted polymers

HNIPs Hollow porous non-imprinted polymers

HPF Heat-processed foods

HPLC/UV High performance liquid chromatography-ultraviolet

HPLC-DAD High performance liquid chromatography-diode array detector

HPLC-DAD High performance liquid chromatography with photodiode array detection

HPLC-FD High-performance liquid chromatography with fluorescence detection

HPLC-MS/MS High-performance liquid chromatography associated with sequential triple quadrupole mass spectrometry

HPLC-VWD High performance liquid chromatography using variable wavelength detection

HP-MINs Hollow porous molecularly imprinted nanospheres

IF Imprinting factor

KH570 3- Methacryloxypropyltrimethoxysilane

SilaneTRAC_2020_56
LOD Limit of detection

LOQ Limit of quantification

MAA Methacrylic acid

MALDI-TOF-MS Matrix-Assisted Laser Desorption/IonizationTime of Flight-mass spectrometry

MCM-41 Mobil Composition of Matter No. 41

MCM-48 Mobil Composition of Matter No. 48

MEFAE Multiphase electrical field assisted extraction

MF Melamine-formaldehyde

MGO Magnetic graphene oxide

MHMIPs Magnetic hollow porous molecularly imprinted polymers

MIPs Molecularly imprinted polymers

MIRs Molecularly imprinted resins

MISG Molecularly imprinted silica gel

MI-SPE Molecular imprinting-based solid-phase extraction

MIT Molecular imprinting technology

MSPD Matrix solid-phase dispersion

MSPE Magnetic solid-phase extraction

mt-MIPs Multi-template MIPs

NIPs Non-imprinted polymers

N-MIPs Nanostructured MIPs

NP Nonyl phenol

PAEs Phthalate esters

PAHs Poly aromatic hydrocarbons

PEG Polyethylene glycol

PF Pre-concentration factor

POSS Polyhedral oligomer silsesquioxanes

PP Precipitation polymerization

PS Polystyrene seed

PTFE Polytetrafluoroethylene

PT-SPE Pipette-tip solid-phase extraction

PT-SPE Pipette tip solid-phase extraction

QuEChERS Quick, Easy, Cheap, Effective, Rugged, Safe

R1 Notoginsenoside R1

RAFT Reversible addition-fragmentation chain transfer

RAFT Reversible addition-fragmentation chain transfer

RAMs Restricted access materials

Rb1 Ginsenoside Rb1

Rg1 Ginsenoside Rg1

RSD Relative standard deviation

SBSE Stir bar sorptive extraction

SPE Solid-phase extraction

SPME Solid-phase microextraction

SPME Solid-phase microextraction

St Styrene

TBZ Thiabendazole

UHPLC-MS/MS Ultra high performance liquid chromatographytandem mass spectrometer

UHPLC-MS/MS Ultra-high-performance liquid chromatography associated with sequential triple quadrupole mass spectrometry

$\mu$-SPE Micro-solid-phase extraction.

WC-TMMIPs Water-compatible, temperature, and magnetic dual-responsive MIPs

$\beta$-CD $\quad \beta$-cyclodextrin 
effect on analytical reliability, and sometimes alteration of analyte into a proper form for the identification or preliminary separation. For enhancing the performance of the sample pretreatment, variant factors, namely selectivity, sensitivity, convenience, cost, and speed have been considered. Depending on the aim of the analysis (quantitative or qualitative), state of the sample (gas, liquid, solid, or semi-solid), property of analyte viz. polarity, solubility, stability, etc. and also the complexity of sample matrices, diverse sample pretreatment methods have been applied such as solvent based extraction [4-6], solid-phase extraction (SPE) [7-9], supercritical fluid extraction [10-12], QuEChERS [13-15], chip based extraction [16-18] and membrane based extraction [19-21]. Parallel to these techniques, the microscale methods have arrived and gained the remarkable tendency owing to lower sample volume consumption, limited losses of analytes, and also saving time, energy and solvents. By overviewing recently published papers in this field, it can be concluded SPE based techniques are more popular compared with others, that may be due to the wide variety of sorbents with different polarity and functionality, high potential for automation, high efficiency, ease of operation, and lower consumption of organic solvents, as well as most importantly multiple modes of SPE. Recently, some review papers have been published about the fundamental and application of mentioned methodologies [22-24]. Fig. 1 illustrates six main types of SPE modes including packed SPE (the conventional SPE and it is commonly expressed as SPE), dispersive SPE (d-SPE), solid-phase microextraction (SPME), pipette-tip SPE (PT-SPE), matrix solid-phase dispersion (MSPD) and stir bar sorptive extraction (SBSE), as well as their respective advantages and disadvantages. Still, some key challenges such as inadequate selectivity and sensitivity have been faced, which have been attempted to address by using innovative advanced functional materials, and thereby extending the applications of SPE.

Molecularly imprinted polymers (MIPs) are tailor-made receptors, inspired from natural antigens and antibodies and proposed by Polyakov in 1931 for the first time [25]. The remarkable selectivity of MIPs arises from the specific cavities, which are created in the polymer network with the memory of the template species (synergy of chemical functionality and stereochemistry). The role of MIPs' precursors, principle of imprinting, different polymerization methods and the pros and cons of each method have been comprehensively reviewed [26-29] and we don't underscore them in the current review. Unique properties of MIPs including ease of preparation, high chemical and physical stability, considerable reusability, low cost, controllable morphology and most importantly high selectivity, make them an excellent candidate for practical applications in different fields such as diagnosis/ drug delivery [30,31], sensors [32,33], catalyst [34,35], chromatography based separation [36,37], and especially sample pretreatment/preparation $[38,39]$. According to the nature of MIPs' precursors, they can be classified into four main groups viz. organic MIPs, inorganic MIPs, bio MIPs, and hybrid MIPs. Free radical polymerization is the most favored method for the preparation of organic based MIPs. Organic functional monomers containing vinyl and allyl groups such as methacrylic acid (MAA), acrylamide (AA), styrene and 4-vinylpyridine (4-VP), and organic cross-linkers like ethylene glycol dimethacrylate (EGDMA), divinylbenzene (DVB) and 1,3-diisopropenyl benzene have been used for synthesis of organic MIPs. Possibility to choose various kinds of functional monomers with different functionality and to choose multiple polymerization routes are the superiority of organic MIPs. Nevertheless, low specific interactions toward analyte, poor analyte diffusion and consumption of a high amount of organic solvents [40] and toxic reagents are the drawbacks of these kinds of materials. It is well-known that the selectivity of MIPs is strongly related to the kind of functional monomers. In this regards, several synthetic functional monomers like ionic liquids [41], deep eutectic solvents [42], pyrrole-phenylboronic acid [43], 2,3,4,6tetra-O-acetyl-glucopyranoside/1,2,3,4,5-pentafluoro-6-

vinylbenzene [44], and alkenyl glycosides glucose [45] have been applied to improve the selectivity of organic MIPs. Commonly, inorganic MIPs are obtained by hydrolysis and condensation of organo silicate or organo titanate precursors in the presence of the imprint molecule in aqueous or alcoholic media. In this type of MIPs, applying the high selectivity of metal coordination to identify and capture macromolecules may be an easy way to imprint the target molecules [46]. Metal coordination with biological molecules is a fast binding process, and the binding strength to stabilize a high efficacy of imprinting with high specificity can be adjusted by choosing appropriate metal ion for a defined template molecule in a media that is simulated to the biological environments [47]. Compared with organic MIPs, inorganic MIPs have higher thermal and mechanical stability, higher affinity and specificity, and faster mass-transfer toward analyte. Moreover, the preparation procedure can be performed in room temperature and eco-friendly reaction solvents such as water and alcohol. However, there are few reagents available for sol-gel reactions, while the design and synthesis of eligible functional monomer are sophisticated as well. To attain both benefits of organic MIPs and inorganic MIPs, several kinds of hybrid MIPs have been synthesized by the combination of acrylic functional monomer and inorganic cross-linker [48-51]. In this type of MIPs, acrylic monomer interacts with template and is linked to the inorganic cross-linker via KH570 bridge. Since the shrinking and swelling degree of inorganic cross-linker is lower than organic cross-linker, higher specificity and analyte diffusion are obtained. Bio MIPs are synthesized by using eco-friendly and green materials, in which specific cavities are generated in the natural polymers or their derivatives network. There are several biomaterials, which can establish a 3D skeleton as well as contain appropriate functional groups to the uptake of template molecule. Chitosan [52], sodium alginate [53], cellulose [54], gelatin [55], and aptamer [56] are multi-functional natural materials frequently applied to fabricate imprinted materials. Although cost-effectiveness, non-toxicity, readily available and facile synthesis condition are notable advantages of bio MIPs, a high degree of swelling causes resizing/deformation of imprinted cavities and subsequent decrease in selectivity. Also, low physical and chemical stability is the other major defect of bio MIPs. Hence, an influential cross-linking of imprinted biomaterials is very essential.

Unfortunately, MIPs have encountered long-term obstacles, namely poor recognition site accessibility, incomplete template removal, slow mass-transfer, low binding capacity, and incompatibility in aqueous media. In this regards, plenty of effort has been dedicated to developing smart strategies for improving MIPs efficiency called molecular imprinting technology (MIT). Recently, some excellent reviews have been published about the application of MIPs in sample preparation and typically have been classified by the analyte of interest or samples [57-60]. Likewise, in the other reviews, the authors have browsed on the development of one category of MIPs such as magnetic MIPs [61,62], sol-gel based MIPs [63,64], nano MIPs [65], monolithic MIPs [66,67] and their applications. To the best of our knowledge, there are few reviews focusing on new strategies for the preparation of MIPs in order to promote their performance and meanwhile their applications in different SPE techniques. In the proposed review, we emphasize on advancement of MIT for the fabrication of supreme MIPs and their particular applications in sample pretreatment and chromatographic separation to enhance the selectivity and sensitivity of analytical approaches, which have been made in the most recent years (approximately 2015-2020). 


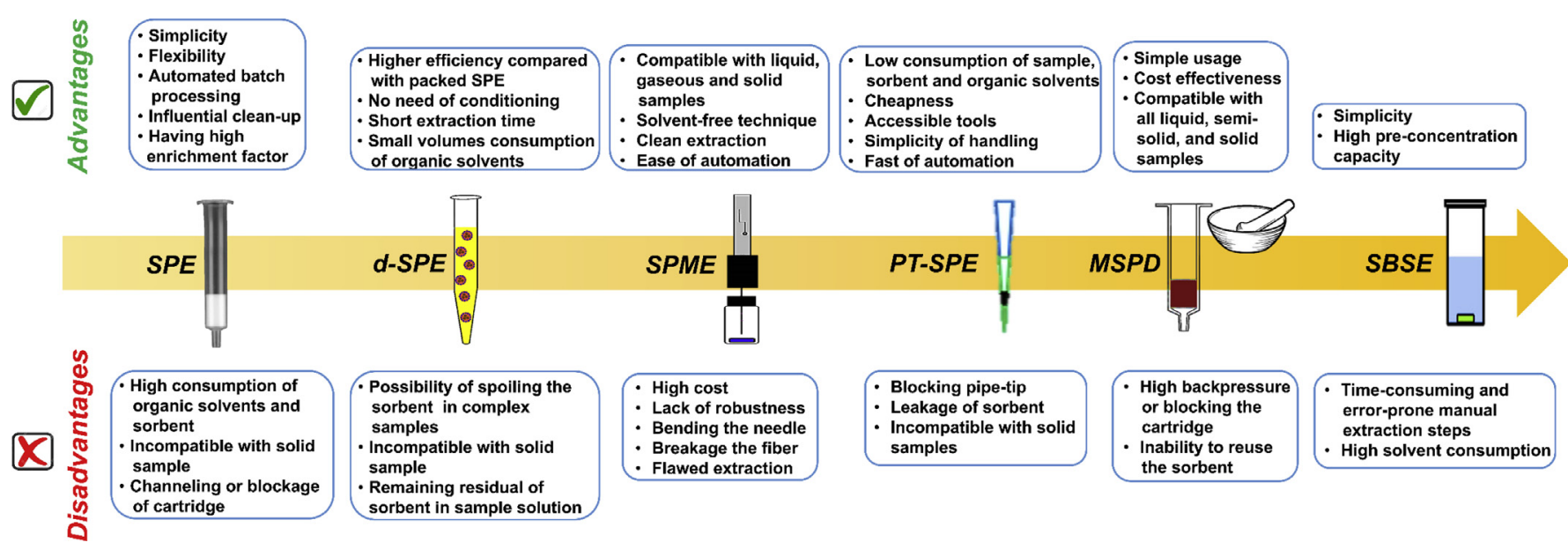

Fig. 1. Different types of SPE modes and their pros and cons.

\section{MIPs based SPE}

MIPs based SPE or molecular imprinting-based SPE, namely MISPE, has been used for many years, and in some cases, some of them have been commercialized; meanwhile, now there is still strong attention to employing them due to their high applicability and throughput. The vast applications of MIPs in all SPE modes are owing to their benefits such as structure predictability, high potential to form a composite with other materials, facile modification, and diverse morphologies such as bulk, micro/nano sphere, membrane and thin film. At the same time, researchers have made a large number of attempts to synthesize preferable MIPs, and innovated the development in the SPE methods for moving towards less steps, simplicity, cost-effectiveness, automation, miniaturization, time saving and environmental friendliness as well as ameliorating figure of merits. For instance, recently, use of MIP phases in miniaturized automated systems, such as in-tube SPME [68] and capillary-LC-MS/MS has acquired abundant attention. Intube SPME allows convenient automation of the extraction process, which not only reduces the analysis time, but also provides higher precision and sensitivity than that of manual off-line techniques [69]. Asiabi and colleagues have prepared a MIP coating by electrochemically controlled in-tube SPME [70]. Applying an electrical potential could improve the extraction efficiency and provided more convenient manipulation of different properties of the extraction system, including selectivity, clean-up, rate, and efficiency. For enhancing selectivity, nanostructured copolymer coating consisting of polypyrrole doped with EGDMA is prepared on the inner surface of a stainless-steel tube by electrochemical synthesis. This is the first report for synthesis of MIP poly (pyrroleEGDMA) polymer by electro-polymerization approach. It is well recognized that the function of MIPs is highly affiliated to the polymerization condition. It is more preferable if the synthesis of MIPs, extraction/purification, and identification process are implemented automatically, least/without human intervention causing minimum/zero errors.

Long sampling/adsorption times, bending of the needle, breakage of the fiber, as well as the high price of fibers, are the weaknesses of SPME, pursuant to which, micro-solid-phase extraction ( $\mu$-SPE) technique is developed. In this technique, the adsorbent or an acceptor liquid phase is held in the membrane so that target species penetrate easily over the membrane pores and are retained by the solid sorbent/acceptor phase; whereas, the diffusion of other components in the sample is hindered by the membrane [71]. This technique can relatively overcome the poor recognition ability of
MIPs in aqueous media, which stems from adsorption of matrix impurities on the surface of MIPs, because direct contact of the sorbent and sample matrix is prevented [72]. With this view, Jayasinghe et al. extracted aflatoxins in fish feed using ultrasound assisted MI- $\mu$-SPE followed by LC-MS/MS [73]. Polypropylene membrane in circle shape protected MIP beads containing integrated imprinted cavities. Owing to elimination of matrix noises, low limits of detection (LODs) within $0.42-1.15 \mu \mathrm{g} \mathrm{kg}^{-1}$ were gained, quite lower than those established by European Commission guidelines for aflatoxins in animal feeds. Although, this variant of SPE has used different kinds of sorbents [74-76], sometimes it cannot minimize the matrix effect even using MIPs [76]. Thus, promotion of new MIPs with high recognition and anti-interferences abilities is much required.

In the following, we will divide innovative approaches in imprinting technologies into ten parts (Fig. 2) and discuss them individually from simpler to more complex, from single to multiple and combined strategies, followed by highlighting the applications in SPE modes for real samples analysis. As schematically shown in Fig. 2, the ten parts of MIPs include monolithic, nano scale, composite, surface imprinting, hollow porous, dummy, multi-functional monomer, multi-template, and water compatible MIPs, as well as restricted access material combined MIPs (RAM-MIPs). Because, the number of published papers in this issue is very enormous, and it is not possible to refer and discuss most of them due to the length of the review, we try to select some representative examples contributed by different research groups and published in different journals, mainly within 2015-2020.

\subsection{Monolithic MIPs}

The first bulk MIPs were introduced in 1931 by Polyakov via imprinting organic molecule in silica matrices [25]. Bulk imprinting is the classical approach for preparation of MIPs. Template, functional monomers, cross-linkers, and initiators are polymerized in a low amount of porogen. After template removal by solvent extraction (for organic MIPs) or calcination (for thermal stable MIPs), followed by crushing and sieving, tiny irregular particles are obtained. For executing MI-SPE, the MIP particles are filled in the SPE cartridge by dry [77] and slurry [78] packing or directly immersed in the sample solution. Before cartridge packing, sedimentation is essential to remove too small particles and preventing cartridge blocking. Also, sedimentation before d-SPE is recommended, because some of too powdery particles can't even be separated from the sample solution by centrifugation. Although bulk imprinting is 


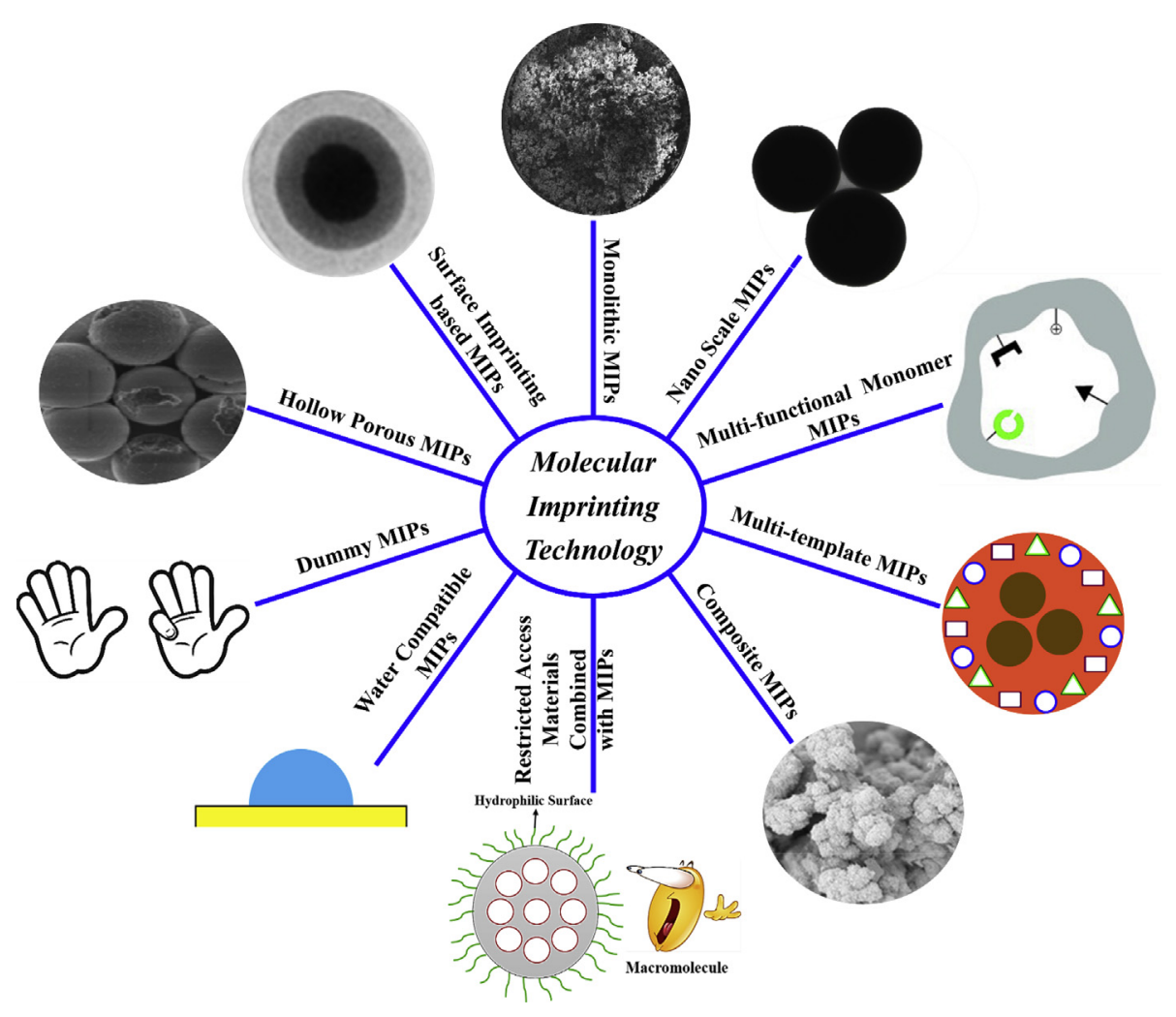

Fig. 2. Novel strategies for molecular imprinting based SPE.

simple, rapid and pure MIPs are provided without any sophisticated or expensive instruments, it suffers from obvious bugs like destroying specific cavities during grounding and lost polymer particles, remaining residual template, low binding capacity and mass transfer, and unsatisfactory resolution, while used as stationary phase in a chromatography column due to the irregularity shape of particles and decreasing the number of theoretical plates. For omitting the post-polymerization step (crushing and sieving) and overcoming its related obstacles, monolithic imprinted materials have been developed swiftly. A monolith is defined as a single piece of a porous material that entirely fills its recipient, usually prepared by in situ reactions [79]. Monolithic MIPs can be facilely synthesized by suitable MIPs' precursor ratios with various forms [66]. In this technique, after blending all MIPs reagents, the liquid mixture is transferred into the desired container such as pipette tip, stainless steel column sealed or other backbones like polyethylene filter $[80,81]$ which is immersed in the MIPs mixture following by keeping in proper temperature for beginning polymerization. In the mentioned approach, the synthesized MIPs don't link to the container or a backbone and the MIPs may be detached by solvent crossing pressure or mechanical impact. By covalent linking the MIPs to the container, physical stability and reusability of monolithic MIPs are significantly amended.

Li et al. prepared monolithic MIPs by in-situ polymerization at the tip of the pipette tip [82]. Tetrapeptide, MAA and EGDMA were used as a template, functional monomer and cross-linker, respectively. Remarkable imprinting factor (IF) more than 30 for the target analyte demonstrated well-done optimization in the synthesis step. Besides, the applicability of their method for selective extraction of enkephalins from human cerebrospinal fluid with good recoveries (85.1-95.7\%) and LODs of 0.05-0.08 nM was proved.

In other kinds of SPE mode, monolithic MIPs were sealed onto the surface of polytetrafluoroethylene (PTFE) disk, and subsequently, the MIP-disk was rotated in sample solution for extracting ecgonine methyl ester from environmental aqueous samples [83]. In the synthesis procedure, firstly PTFE disk was activated by FluoroEtch ${ }^{\circledR}$ solution, secondly, the vinylization of the disk was accomplished for proper attaching of the MIPs over disk surface, and finally, polymerization was carried out by using MAA, EGDMA, AIBN and ecgonine methyl ester template. The developed extraction device exhibited an extraction phase with a high surface areato-volume ratio, and it can be magnetically stirred (along the vertical axis) at higher rates without damaging the solid phase. The adsorption experiments displayed the maximum adsorption capacity of $17.7 \mu \mathrm{g} \mathrm{g}^{-1}$ for MIP-disk that is routine for monolithic MIPs, as well as the IF value was as excellent as 25 . Analyte determination was performed by both ion mobility spectrometry and UHPLC-MS/MS; the results revealed the attained LOD by UHPLCMS/MS (13 $\left.\mathrm{ng} \mathrm{L}^{-1}\right)$ was approximately 6 times lower than ion mobility spectrometry ( $75 \mathrm{ng} \mathrm{L}^{-1}$ ).

In contrast with organic MIPs, fabrication of inorganic MIPs is difficult due to easily cracking and breaking of the inorganic MIPs during preparation. In sol-gel reaction, after condensation, aerogel converted to xerogel by keeping in relatively high temperature (usually $60^{\circ} \mathrm{C}$ ). By increasing the temperature, trapped porogen molecules into the polymer skeleton were evaporated and this phenomenon causes disintegrating monolithic structure. For improving mechanical stability and prevent cracking, Arabi et al. added thiourea to sol-gel MIPs' reagents followed by in-situ polymerization in pipette-tip for avoiding cracking the monolithic [84]. Besides, the ratio of monomer ((3-Aminopropyl)trimethoxysilane) and cross-linker (tetraethyl orthosilicate) and the temperature for drying the gel were optimized to obtain homogeneous permeable imprinted monolithic. It should be pointed out that the permeability of monolithic MIPs is essential since inadequate permeability in PT-SPE or monolithic in chromatography column leads to unfavorable mass-transfer and binding capacity and likewise too high backpressure. Finally, they applied inorganic monolithic MIPs 
for clean-up and enrichment of gallic acid from orange juice samples.

It is always important to develop automatic sample preparation because of some remarkable advantages such as performing the entire analysis under controlled conditions as well as minimizing human and systematic errors. By this aim, porous monolithic MIPs were synthesized in $100 \mu \mathrm{m}$ inner diameter fused-silica capillary and then coupled to the nano-liquid chromatography for determination of cocaine and its metabolite, benzoylecgonine, in human plasma and saliva samples [85]. In contrast with common HPLC, nano-liquid chromatography requires less time, samples, and solvents and is more eco-friendly. However, the miniaturization of the analytical instrument may cause lower resolution owing to the reduction of separation lengths. For monolith preparation, porogen type is a crucial variable that could allow the tuning of the polymer porosity without changing its final chemical composition. The permeability of MIPs $\left(1.2 \times 10^{-15} \mathrm{~m}^{2}\right)$ was higher than that of nonimprinted polymers (NIPs) $\left(9.1 \times 10^{-15} \mathrm{~m}^{2}\right)$, which was due to the remaining more pores in MIPs generated by template removal. The IF values were 3.9 and 2.2 for cocaine and benzoylecgonine, respectively, demonstrating imprinting can be successfully implemented in the very thin capillary [86]. Recoveries more than $88.6 \%$ along with relative standard deviations (RSDs) lower than $11.1 \%$ without significant matrix effect for both plasma and saliva samples were worthy achievements.

For enhancing molecular recognition, Yang et al. prepared monolithic MIPs for S-naproxen with the assistance of polyhedral oligomeric silsesquioxanes (POSS) [87]. POSS is a cage-like nanoscale (approximately $1.5 \mathrm{~nm}$ ) silica based material with the molecular formula of $\mathrm{Si}_{8} \mathrm{O}_{12}$, which is easily anchored to polymer chain and can impose the hindrance to the spin of the part of the parent polymer molecule. During the synthesis route, porous MCM-41 was added as a scaffold, and 1-propylmethacrylateheptaisobutyl substituted as POSS monomer to typical reagents, thus dipole interaction among the POSS could barrier the molecular chain movement and enhance the specificity (Fig. 3). IF of 6.86 was the advantage of the developed method, but the adsorption capacity of $3.2 \mathrm{mg} \mathrm{g}^{-1}$ was not a remarkable achievement.

Monolithic MIPs can be applied in the SPME technique as well. 1-Ally-3-vinylimidazolium chloride ionic liquid, and 3,4-dihydroxy benzenepropanoic acid (DBA), were chosen as functional monomer and template, respectively, for creation of monolithic MIPs on the

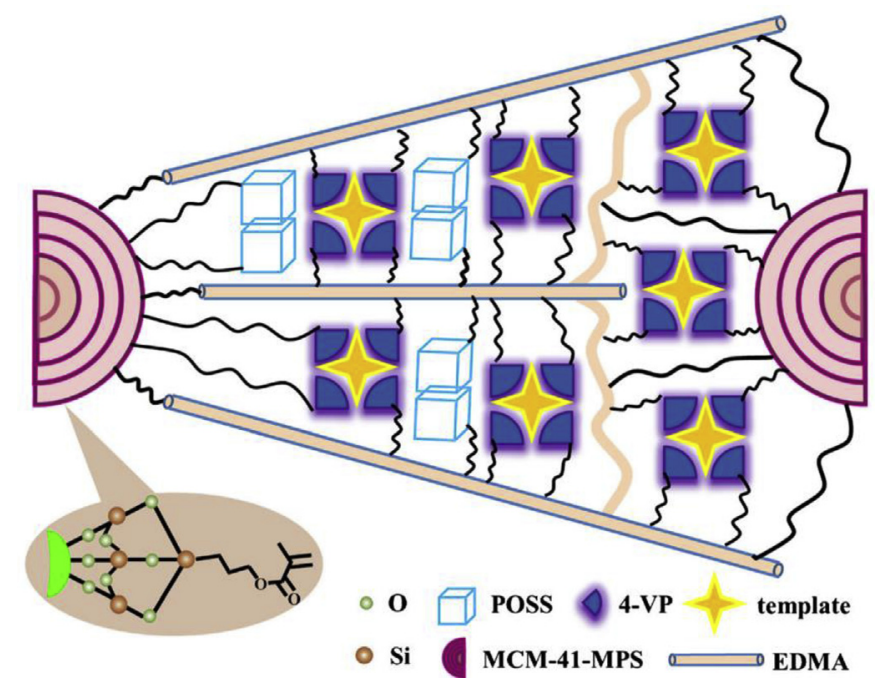

Fig. 3. Mechanism of monolithic MIPs containing POSS by dendritic effect of MCM-41 scaffolds. Reproduced with permission [87]. Copyright @ $\odot 2018$ Springer. fiber [88]. Under the optimized preparation conditions, the recognition coefficient of PIL-MIPs for DBA was as high as 11.6. The prepared MIP-SPME had a high potential for extraction of five phenolic acids (PAs) in fruit juice and beer samples. As can be seen in the HPLC-DAD chromatograms (Fig. 4), there were several interfering compounds owing to the complicated matrices of fruit juice. After treating fruit juice samples with MIP-SPME, interfering substances' peaks disappeared, suggesting high selectivity and anti-interference ability of the prepared MIP-SPME. Meanwhile, because of specific recognition sites in monolithic MIPs, the peak heights of all analytes achieved with MIP-SPME were much higher than that of NIP-SPME. The maximum wavelength for target analytes were different and the measurements were performed in varying suitable wavelength to gain highest sensitivity. The obtained LODs for PAs in fruit juice $\left(0.024-0.24 \mu \mathrm{g} \mathrm{L}^{-1}\right)$ and beer sample $\left(0.011-0.052 \mu \mathrm{g} \mathrm{L}^{-1}\right)$ were much lower than that of nonMIPs methods, which proved the supreme applicability of MIPs for preconcentration of analytes from complex samples.

The monolithic MIPs are qualified for trapping peptides as well. Liu et al. focused on the optimization of hydrophilic melamineformaldehyde (MF) monolithic polymers followed by binding/ rebinding phosphorylated amino acids and peptides as target analytes from partly aqueous media [89]. Briefly, they prepared aqueous precondensates of melamine and formaldehyde at alkaline media in bulk phase, and subsequently mixed porogens and diluents, and finally filled in capillaries and curing. Recognition and separations of phosphorylated peptides were successfully carried out, implying that MF monolithic MIPs are an ideal candidate for clinical diagnosis. However, their method performance, extremely depends on monolithic properties, so the optimization process of the porogen composition should be performed to arrive at similar porous properties. In the further work of this group, they developed the synthesis tactic to overcome the mentioned defect by polymerizing an appropriate monolithic substrate with well-defined porous properties and attaching groups on its pore surfaces, followed by grafting a thin MIP layer with the aid of these groups [90]. They prepared monoliths in the capillary format by trimethylolpropane trimethacrylate (TRIM) core on which MIP shell was decorated through oriented photografting of a variety of mono- and bis-imidazolium host monomers at subzero temperature, using six different continuous or pulsed UV light sources. By comparing the $\mu$-LC chromatograms of a mixture of human Angiotensin II octapeptides on the different capillary monoliths (Fig. 5), it can clearly see that bare TRIM monolith lacked recognition capability and displayed minimal nonspecific interactions. The NIPs displayed a comparatively longer analyte retention time and a slight discrimination between the amino acids. On the MIP monolith, all target species were separated with acceptable resolutions and retention time, which was due to successful imprinting effect.

Aptamers (Apts) are oligonucleotide or peptide molecules can act as ligand and specifically bind with chemo/bio target analytes with high affinity [91]. Both Apts and MIPs are applied to reduce non-specific adsorption in sample preparation. A doublerecognized aptamer-molecularly imprinted (Apt-MI) monolithic column for high-specificity recognition of ochratoxin A (OTA) was constructed [92]. The hydrogen-bond interactions between the amine groups of functional monomer (AMPS) and the chlorine atoms of OTA could increase specific adsorption of OTA on Apt-MI monolithic column. At different mobile phase velocities, the prepared monolithic column proved the good mechanical stability.

$\mathrm{Xu}$ et al. fabricated gold nanoparticles@Apt-based affinity monolithic column for high-efficient online specific discrimination of zearalenone in cereals [93]. Most of the Apt-based monoliths are commonly prepared by using acrylate monomers which have a limited Apt coverage density. To address this limitation, in this 


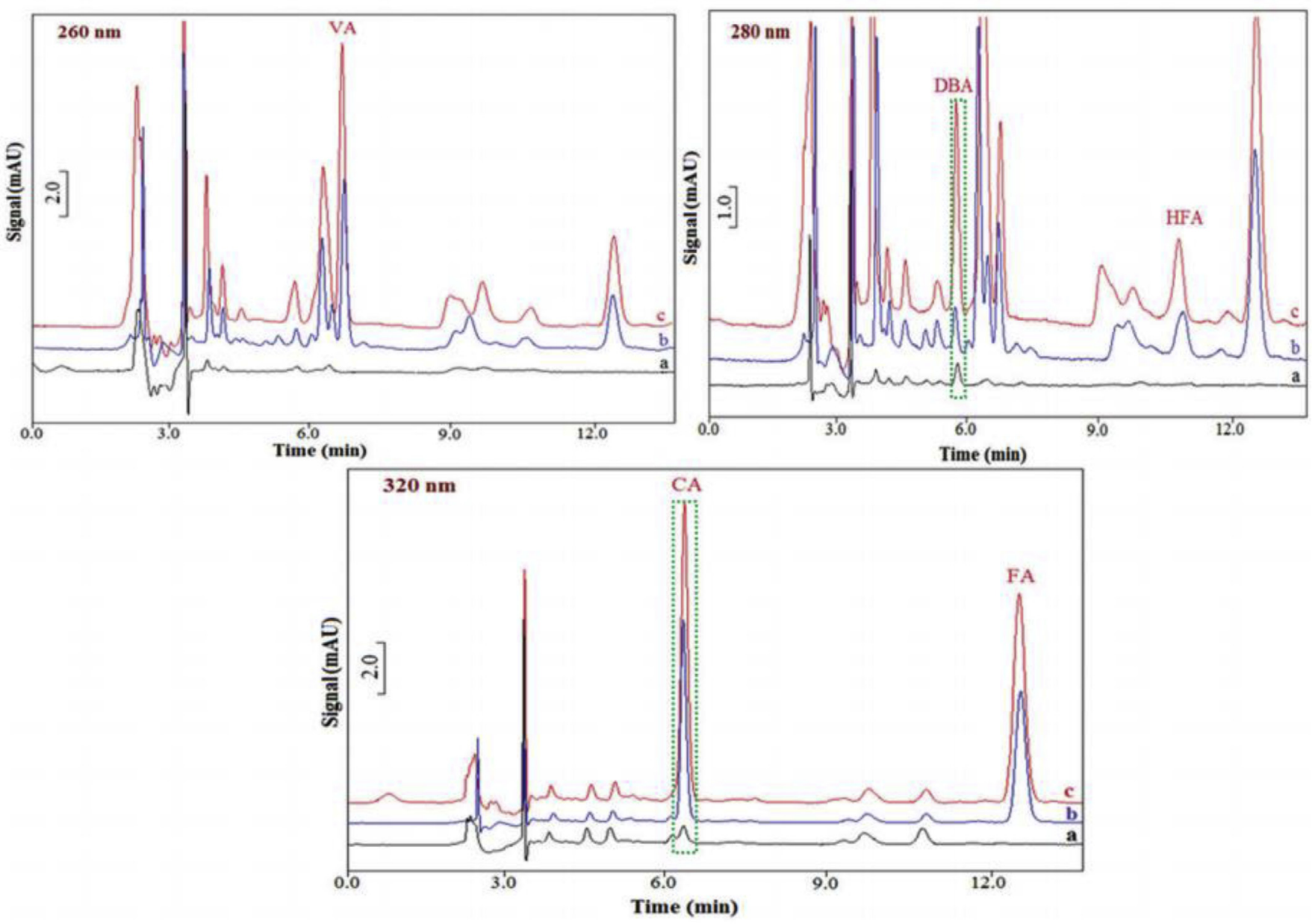

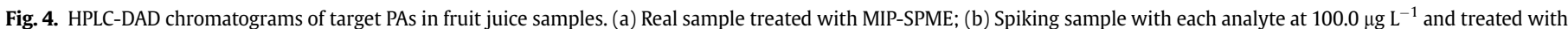
NIP-SPME; (c) Spiking sample with each analyte at $100.0 \mu \mathrm{g} \mathrm{L}^{-1}$ and treated with MIP-SPME. Reproduced with permission [88]. Copyright $\odot$ 2017 Royal Society of Chemistry.

study, siloxane-based hybrid monolith was prepared which showed higher surface area, framework rigidity and also higher Apt coverage density (3636 pmol $\mu \mathrm{L}^{-1}$ ).

Monolithic MIPs as chromatography column packing have been continuously synthesized and applied for selective separation of various small molecules. However, these columns cannot compete with commercial common columns because of more peak broadening and tailing, which are corresponding to the heterogeneity of the binding sites. Thus, instead of non-covalent imprinting, by covalent imprinting approach monolithic MIPs with higher affinity and homogenous binding sites can be constructed. Regardless of the peak broadening, monolithic MIPs have also a great potential for separation of biomolecules and enantiomers [94,95].

Several main impediments of bulk imprinting still exist in monolithic MIPs, including specific cavities locating too deep inside the polymer network that hinders mass-transfer of the analyte, and low elution of template which causes low binding capacity. To overcome these problems, the MIPs particles have been synthesized in nano-scale.

\subsection{Nano scale MIPs}

Producing of nanostructured MIPs (N-MIPs) have been done by the combination of nanotechnology and MIT, interestingly improving the characteristics of MIPs compared with monolithic and bulk MIPs. The traditional MIPs are not very applicable in sensing and separation techniques and also more challenging for in-vivo applications, drug delivery and biomedical imaging. These defects are related to the size of bulk and monolithic MIPs which have irregular size distribution, low binding capacity, incomplete template removal and slow mass transfer. By changing MIPs' precursors ratio of bulk imprinting (such as porogen volume) and/or polymerization conditions (like stirring speed, or using capping agents), N-MIPs can be attained with higher specific surface area and nanoscale particle size. In 1999, the first N-MIPs were prepared via precipitation polymerization by Mosbach's group [96]. N-MIPs can be obtained by different polymerization routes such as emulsion polymerization, self-assembly imprinting, and solid-phase imprinting. The solvent molecules can easily penetrate to small MIP particles and elute template species sufficiency, and subsequently the binding capacity, will be enhanced as well as accessibility of target analyte improved with higher binding kinetics [97,98]. N-MIPs with diameters typically below $200 \mathrm{~nm}$ have high compatibility with various nanodevices and wide applications in vivo/vitro biomedical field [99].

With this outlook, Arabi et al. prepared N-MIPs by precipitation polymerization, and then applied them for the uptake of celecoxib from human plasma samples [100]. They investigated three different porogens (DMSO, ethanol, acetonitrile) and found that acetonitrile provided the highest adsorption capacity of $85.47 \mathrm{mg} \mathrm{g}^{-1}$. The type of porogen has a substantial influence on the pore size and surface area of MIPs. For optimization of extraction 

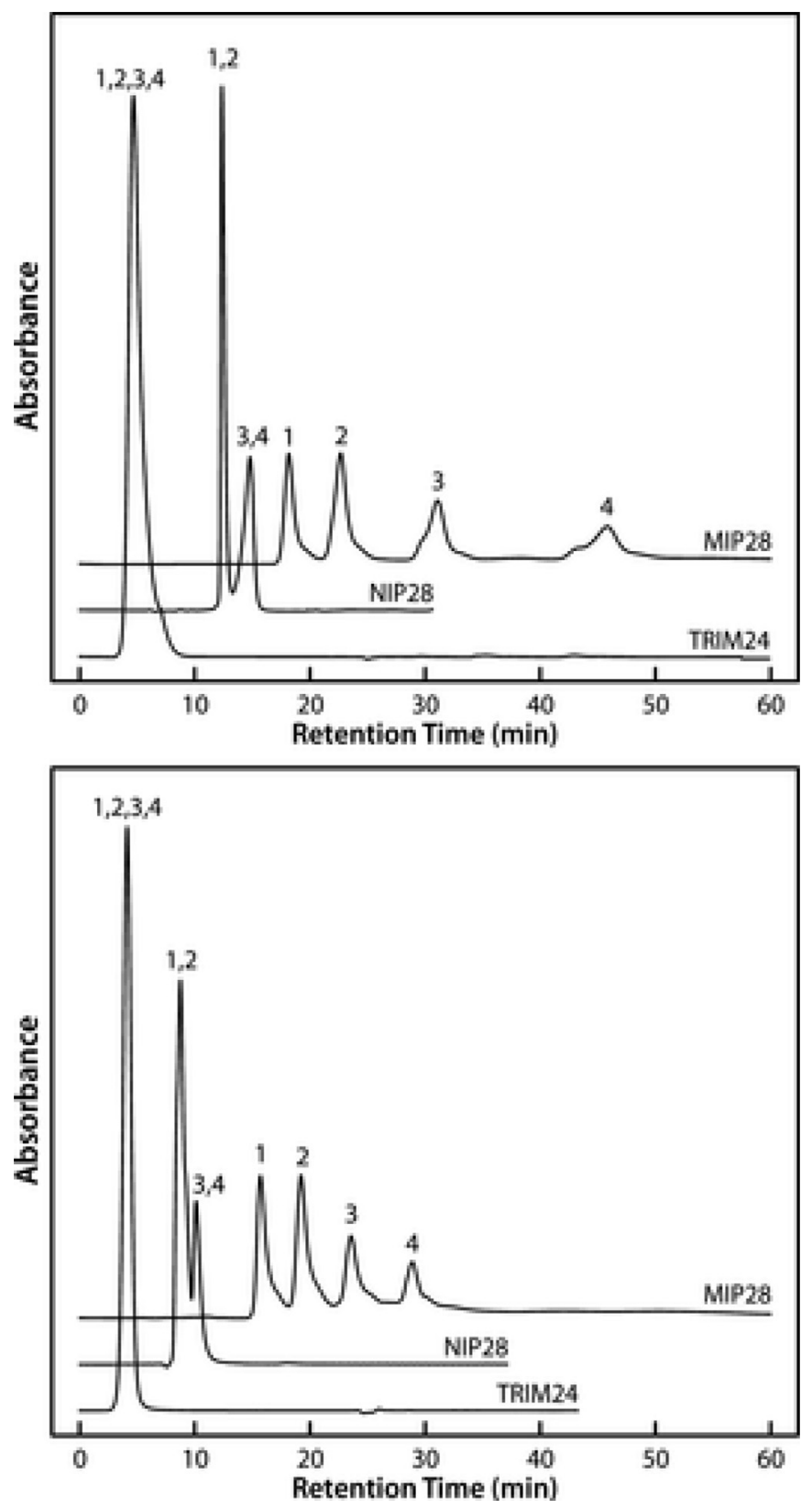

Fig. 5. $\mu$-LC chromatograms of a mixture of human Angiotensin II octapeptides on the capillary monoliths. Peptide peaks: DRVSIHPF (1); DRVYIHPF (2); DRVpYIHPF (3); DRVpSIHPF (4). Mobile phases: Top, ACN/H $\mathrm{H}_{2} \mathrm{O}(0.1 \%$ TFA; $90: 10, \mathrm{v} / \mathrm{v})$; bottom, ACN/ $100 \mathrm{mM}$ aqueous HEPES buffer, pH $7.4(10: 90, \mathrm{v} / \mathrm{v})$; flow rate, $4 \mu \mathrm{L} / \mathrm{min}$; UV detection at $210 \mathrm{~nm}$; injection volume, $35 \mathrm{~nL}$. Reproduced with permission [90]. Copyright (๑) 2019 American Chemical Society.

variables, experimental design methodology was used to ensure attaining a true optimum level. The developed N-MIPs-HPLC/UV exhibited satisfactory recoveries more than $96 \%$ and low LOD of $0.08 \mu \mathrm{g} \mathrm{L}^{-1}$. In addition, the N-MIPs could be reused at least 11 times.

The high binding capacity of N-MIPs makes them a good candidate for drug load/release as well. N-MIPs with a size of approximately $66 \mathrm{~nm}$ by single-stage process miniemulsion polymerization were synthesized and used for extracting and releasing olanzapine [101]. Adsorption experiments revealed that the maximum binding capacity was $95 \mathrm{mg} \mathrm{g}^{-1}$ and in compliance with the Langmuir model. Besides, in vitro release experiments in different media were performed and the application of N-MIPs in
SPE method prior to HPLC/UV provided $0.18 \mu \mathrm{g} \mathrm{L}^{-1}$ and $0.39 \mu \mathrm{g} \mathrm{L}^{-1}$ LOD and LOQ, respectively.

For better comprehending the effect of the size of N-MIPs, Cenci et al. examined the efficiency of two micro and nano MIPs for epitope NR11 of cardiac troponin I and CK13 of human serum by MALDI-TOF-MS [102]. Their work was consisted of loading of the MIPs in peptide solution for $30 \mathrm{~min}$, washing the MIPs for removing interferent compounds followed by MALDI-TOF-MS analysis. Experimental results demonstrated that significant improvement on $\mathrm{S} / \mathrm{N}$ ratio was observed when N-MIPs were applied for recognition of the target protein.

Also, inorganic MIPs can be synthesized in nano scale. Imprinted silica nanoparticles were prepared by the sol-gel route and employed as dispersant sorbent in MSPD method for the uptake of acrylamide (AA) from bread and biscuit samples [103]. AA is a hazardous hydrophilic compound and carcinogenic for humans, and high content of AA is found in heat treated carbohydrate-rich foods. Since, the sorbent was hydrophilic and contained specific cavities complementary with AA, it was able to easily uptake AA from samples during blending and homogenizing in a mortar without matrix interferences. In addition, for better homogenization of the mixture of sample and dispersant sorbent, and decreasing cartridge backpressure, sea sand was added. Simple extraction protocol, direct extraction of analyte from solid samples, low waste generation, high recovery and good precision (83.7-99.3\%, and RSD $\leq 6.72 \%$ ) were the benefits of this work.

Metal organic frameworks (MOFs) are versatile functional materials with high chemical and physical stability and adsorption capacity. UiO-66, a kind of porous MOFs with cubic structure, contains $\mathrm{Zr}$ cluster and benzene-1,4-dicarboxylic acid ligand which link through coordination bonds. Owing to the strong $\mathrm{Zr}-\mathrm{O}$ bonds, UiO-66 possesses excellent thermal stability and broad solvent resistance and suitable functional sites to interact with various analytes [104]. Three hydrophobic amino acids with different physical/chemical properties were applied to in situ tailor defects in UiO-66 through targeted incoordination of missing linkers or missing nodes [105]. The obtained MOFs lengths ranged from 1.8 to $3.1 \mathrm{~nm}$, which was predicted by molecular simulation. The high recognition and selectivity of imprinted MOFs have demonstrated these materials are qualified delivery tools for high throughput screening of bioactive peptides. Recently, imprinted MOFs have attracted remarkable interest since these novel substances benefit from advantages of both MIPs and MOFs.

Analysis of chiral compounds is challengeable and needs to develop modern analytical chemistry methods. A number of researches on applications of MIPs in chiral separations and analysis have been reviewed in 2018 [95]. The use of MIPs for enantioseparation was first reported as early as 1978 [106]. In recent years, various MIPs have been increasingly applied for determination of chiral compounds. For example, lately in 2020, Liu et al. reported a kind of new MIPs synthesized by using a functionalized initiator for chiral-selective recognition of propranolol [107]. That is, carboxyl-functionalized radical initiator was utilized to replace traditional functional monomer. The isotherm model obeyed the Freundlich model which showed the heterogeneous binding sites of MIPs, and the MIPs were reusable for at least five times without losing its performance.

Although, often the specific surface area and binding capacity of N-MIPs are more preferable than bulk and monolithic MIPs, some challenges for practical applications in different SPE modes are still facing, mainly related to the size of N-MIPs. Separating N-MIPs from solution needs centrifugation or filtration. This may not be laborious on a lab scale, but on a larger scale, it requires a great deal of cost and energy. In this regards, other functional materials can be utilized to assist improving MIPs' characteristics like specific 
surface area, binding capacity, and functionality. Hence, composite MIPs have been widely used to modify MIPs' performance.

\subsection{Composite MIPs}

The combination of MIPs and other functional materials supplies versatile composites with promising advantages of both. The composites also named composite MIPs can be designed according to different purposes, including improving the physical and chemical stability of MIPs, functionalization of proposed MIPs, increasing specific surface area and enhancing adsorption capacity of proposed MIPs, etc. Up to now, common nanomaterials such as graphene, $\mathrm{Fe}_{3} \mathrm{O}_{4}$ and other metal nanoparticles, quantum dots, polystyrene, $\mathrm{TiO}_{2}, \mathrm{MOF}$ and chitosan have been successfully incorporated with MIPs and modified the performances in different SPE modes. Graphene has high specific surface area (about $2630 \mathrm{~m}^{2} \mathrm{~g}^{-1}$ in a single sheet), which is higher than that of activated carbons and carbon nanotubes, and also it has excellent thermal and mechanical strength which makes it a worthy candidate for constructing MIP composites [108]. $\mathrm{Fe}_{3} \mathrm{O}_{4}$ nanoparticles are commonly synthesized through precipitation or hydrothermal methods. By precipitation route, the size of obtaining $\mathrm{Fe}_{3} \mathrm{O}_{4}$ is approximately lower than $50 \mathrm{~nm}$ with the magnetic saturation about $60 \mathrm{emug}^{-1}$. In hydrothermal method, although the obtained $\mathrm{Fe}_{3} \mathrm{O}_{4}$ has a larger size (about $500 \mathrm{~nm}$ ), the magnetic saturation is about 80 emug $^{-1}$. Compared with hydrothermal method, precipitation method is greener, in which synthesis steps are performed in alkaline aqueous media under nitrogen protection. Whenever composites contain magnetic components, applying an external magnetic field can easily separate the composites from solution without needing filtration or centrifugation. Howbeit, this step must be done carefully, since entering solid particles along with eluent to chromatography column lead to emerging ghost peaks and/or splitting the peaks. Thus, high saturation magnetic value assists complete separating magnetic materials and no particles are remained in the eluent. It is well-known that the adsorption performance of MIPs has a close relationship with polymer morphology. Since, surface imprinting based MIPs can be classified as composite MIPs and in the next section we will discuss them, in the current section we try to mention composite MIPs excluding surface imprinting based MIPs.

MIPs can interact with other functional materials through covalent [109] and non-covalent interactions [110] such as hydrogen bonds, Van der Waals forces, ion or hydrophobic interactions. Luo et al. prepared composite MIPs by covalent linking of graphene sheets, $\mathrm{Fe}_{3} \mathrm{O}_{4}$ nanoparticles, and silica based imprinted network and then applied them for the uptake of 4-nitrophenol from water samples by the d-SPE method [111]. Graphene sheets have two dimensional nanostructure with large surface area and numerous delocalized $\pi$-electrons that effectively interact with target analytes containing benzene ring through $\pi-\pi$ assembling. The composite gained distinguished benefits viz. saturation magnetic value of 30.49 emu $\mathrm{g}^{-1}$, large binding capacity $\left(142 \mathrm{mg} \mathrm{g}^{-1}\right)$, good selectivity and very fast adsorption kinetics ( $2 \mathrm{~min}$ ). The magnetic property of prepared composite decreased extraction time and enhanced reusability and stability of the sorbent.

Magnetic composites are excellent candidates for developing traditional SBSE. The principle of SBSE is approximately the same as SPME, but SBSE needs a larger amount of sorbents and they are coated on the surface of a magnetic stir-bar instead of silica fiber of SPME. Unfortunately, preparation procedure of magnetic stir-bar is extremely time consuming and tedious, including etching glass support by a large amount of solvent, $\mathrm{NaOH}$ treatment to activate silanol groups, and silanization for linking MIPs to the glass. DíazÁlvarez and co-workers reported a new variant of SBSE relying on the raspberry-cake like magnetic monolithic MIPs [112]. First, $\mathrm{Fe}_{3} \mathrm{O}_{4}$ nanoparticles were modified with oleic acid. Then, the modified $\mathrm{Fe}_{3} \mathrm{O}_{4}$ nanoparticles were encapsulated inside a vinyl containing silica network by sol-gel approach and finally the MIP network embraced modified $\mathrm{Fe}_{3} \mathrm{O}_{4}$ nanoparticles via covalent bonds. In their method, no magnet or glass support is needed, since acrylic based monolithic MIPs contained $\mathrm{Fe}_{3} \mathrm{O}_{4}$ nanoparticles and could directly be immersed in the sample solution. By this technique, the contact surface of sorbent and sample solution was enhanced; so the mass transfer of analyte was facilitated. During extraction procedure, acetonitrile and methanol: acetic acid $(85: 15, v / v)$ were used as rinsing solvent for washing matrix interferences and elution solvent for removing analytes, respectively. After optimization, the prepared composite successfully extracted and enriched thiabendazole (TBZ) and carbendazim (CBZ) from orange samples. The calculated LODs were 0.13 and $0.10 \mathrm{mg} \mathrm{kg}^{-1}$ for CBZ and TBZ, respectively, low enough to a desirable analysis of both analytes in orange samples. Finally, the authors suggested improvements in the chemical and mechanical stability of stir-bars should be considered for the future works.

Huang et al. constructed $\mathrm{Ag} /$ graphene oxide-dual-templates MIPs (Ag/GO-dual-MIPs) in a small glass capillary chip and introduced it as a new sample pretreatment method for selective extraction of bisphenol A (BPA) and nonyl phenol (NP) from fish samples [113]. The authors claimed that by incorporating $\mathrm{Ag}$ nanoparticles with $\mathrm{GO}$, the physicochemical performances of the composite were dramatically improved. Due to the distinct structure and functionality of BPA and NP, they were both used for preparation of $\mathrm{Ag} / \mathrm{GO}$-dual-MIPs to attain higher selectivity. Fig. 6 indicates the preparation steps of PDMS/glass chip with microchannels. By CAD software the channels were sculptured on a copper mold, and PDMS oligomer was appropriately mixed with curing agent and cured to generate a PDMS layer. Afterward, MISPE monolithic capillary columns were implanted into the PDMS layer and finally PDMS/glass chip coupled with MI-SPE monolithic capillary columns was constructed. The proposed miniaturized extraction method allowed stronger adsorption volume and higher throughput parallel processing. Satisfactory precisions less than 5.1\%, and high enrichment factors 113 and 92 for BPA and NP, respectively, implied feasibly applicability for simultaneous extraction of BPA and NP from complex fish samples. Regardless of benefits, the proposed method suffered from laborious steps for designing the chip, existence of dead volume in channels, and the composite maybe leak after several usages owing to lack of stable chemical bond between composite and chip wall.

MOFs based composites are other type of materials with excellent versatility and applicability. The defects of MIPs such as low specific surface area, porosity, and mass-transfer can be interestingly prevailed by connecting MIPs to the MOFs [114]. Selection criteria of kind of MOFs is generally depends on the stability, synthesis conditions, nature of target analyte and application purpose. More recently, various chemicals such as phthalates in water [115], aflatoxin B1 in peanut [116], rhodamine B in chili powder [117], and hydrochlorothiazide in human urine [118] were selectively extracted by composite MIPs. Despite of the prominent efficiency of composite MIPs, several drawbacks still happen occasionally, including leakage of residual template, undesirable binding capacity and low mass-transfer, limiting related applications, and need to be overcome by other strategies.

\subsection{Surface imprinting based MIPs}

Generally, three major problems are associated with micro sized MIPs: (i) difficulty of eliminating template molecules from interior binding sites; (ii) the rebinding capacity is limited by the small 

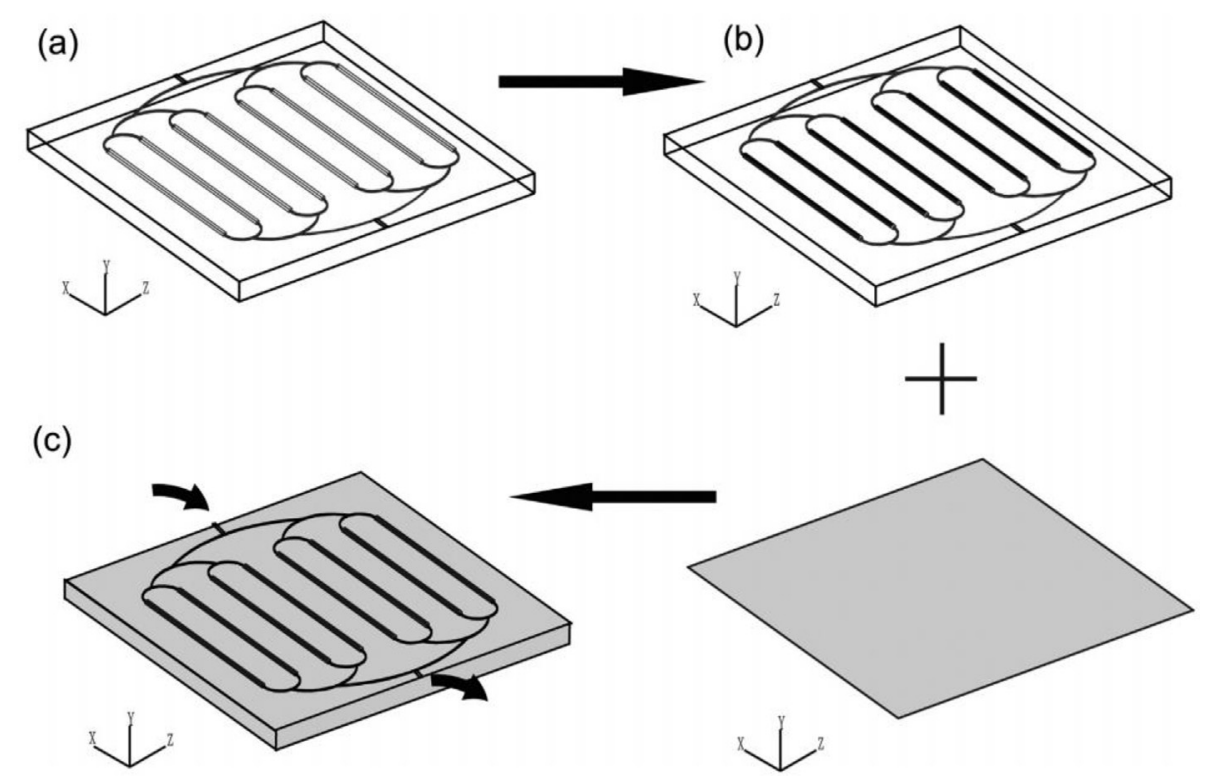

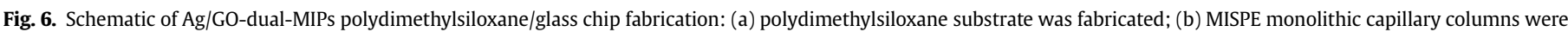

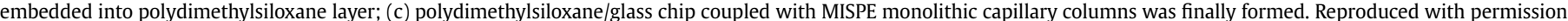
[113]. Copyright ๑ 2016 Elsevier.

number of binding sites on/near the surface; and (iii) target molecules are easily hindered from accessing binding sites deep in the interior of the particles. Besides, the adsorption kinetics of the target analyte by MIPs is low mostly. This phenomenon can be explained by the adsorption process of MIPs, which has two main steps: first, mass-transfer of analyte from the sample solution to the surface of MIPs, and second, penetration of the analyte from surface into the specific cavities. As analyte should be matched to the cavities, this event takes long. That is, in order to access the deeper cavities in MIPs' skeleton, analyte needs more time to penetrate to recognition sites thereby has slower binding kinetic. Hence, usually adsorption kinetics for NIPs is faster than MIPs because of the absence of specific cavities in NIPs' network and non-specific adsorption of most analyte molecules on the surface of NIPs. To address the mentioned defects and increase mass-transfer, binding capacity and sorption kinetics, surface imprinting based MIPs have been vastly utilized. This strategy was first reported by Mosbach's group [119]. In this technique, a thin layer of MIPs is grafted on the surface of different kinds of functional supports such as $\mathrm{SiO}_{2}$ nanoparticles, carbon nanotubes, metal oxide particles, carbon spheres, MOFs, quantum dots and so on. High specific surface area, stability in harsh conditions, and containing suitable functionality, are the factors that are considered for selecting support materials. With respect to type of polymer reagents, various grafting techniques have been applied for anchoring imprinted layer including free-radical grafting [120], living polymerization (atom transfer radical polymerization (ATRP) [121], reversible additionfragmentation chain transfer (RAFT) [97,122]), and photochemical grafting [123]. If detail of each technique is needed, a comprehensive review contributed by Bhattacharya and Misra is recommended for reading [124]. Concerning thermal stability, abundant hydroxyl functional groups for further modification, mild synthesis conditions, and ultimately furnishing MIPs with a magnetic property, $\mathrm{Fe}_{3} \mathrm{O}_{4}$ nanoparticles are widely used as favorable core. For example, our group synthesized eco-friendly dummy MIPs (DMIPs) based on green approach, for magnetic SPE (MSPE) of acrylamide from biscuit samples [125]. Since $\mathrm{Fe}_{3} \mathrm{O}_{4}$ nanoparticles are unstable and easily oxidized in acidic condition, first, they were decorated with polyethylene glycol chain (PEG), and then chitosan bio- polymer grafted $\mathrm{Fe}_{3} \mathrm{O}_{4} @$ PEG in aqueous media, in which dummy template molecules (propanamide) were embedded in the structure. Biscuit contains a high amount of long chain fatty acids, which can seriously interfere with acrylamide's peak in HPLC $C_{18}$ column. By considering the principle of green chemistry, we used ice bath instead of toxic nonpolar (hexane) washing solvent for removing fatty acids. After loading step, the sample solution was put in an ice bath to the solidification of fatty acids and easily separated from solution. The developed method exhibited a good linearity over the wide range of $5.0-5000.0 \mu \mathrm{g} \mathrm{kg}^{-1}$ and LOD and LOQ of $1.3 \mu \mathrm{g} \mathrm{kg}^{-1}$ and $4.4 \mu \mathrm{g} \mathrm{kg}^{-1}$, respectively.

Hydrophilic magnetic molecularly imprinted resins (MIRs) were designed by copolycondensation of resorcinol, melamine, and formaldehyde in the surface of modified $\mathrm{Fe}_{3} \mathrm{O}_{4}$ [126]. The thickness of the imprinted layer was controlled to locate binding sites near the surface of the sorbent and the MIRs with the imprinted layer thickness of $5 \mathrm{~nm}$ gained maximum efficiency. The prepared MIR sorbent possessed a high affinity toward seven benzoic acids and could enrich target analytes from aqueous samples with worthy recoveries in the range of $81.8-108.7 \%$. Also, the sorbent could be reused for ten times that is cost-effective.

In the other work, $\mathrm{Fe}_{3} \mathrm{O}_{4}$ particles were coated by carboxylated cellulose layer, followed by covering with MIPs layer using fluoroquinolone as a template [127]. Satisfactory adsorption capacities (1.3-7.24 $\mathrm{mg} \mathrm{g}^{-1}$ ) and IF values (0.96-2.83) were obtained for target analytes. The sorbent was dispersed into the homogenized egg sample and the extraction adsorption time took long for $80 \mathrm{~min}$ to extract six fluoroquinolones. Certified figures of merit for HPLC analysis, namely $75.2-104.9 \%$ recoveries and $3.6-18.4 \mathrm{ng} \mathrm{g}^{-1}$ LODs, implied high throughput of the method.

MIL-101 MOF is an eligible core and was employed by Liu et al. [128]. For MIP preparation, they adopted the dummy imprinting strategy (it will be specifically discussed in section 2.6. Dummy MIPs) by selecting pyrrolidine-3-carboxylic acid as a dummy molecule for pyrraline, due to the lower cost and chemical structure similarity with the target analyte. Fig. 7 shows the schematic of the synthesis method (A) and SEM images (B) of the MIL-101@DMIP. As seen in Fig. 7B (b), after polymerization, the MIL-101 MOF was fully encapsulated and had an average size of $400-600 \mathrm{~nm}$. The analysis 
A
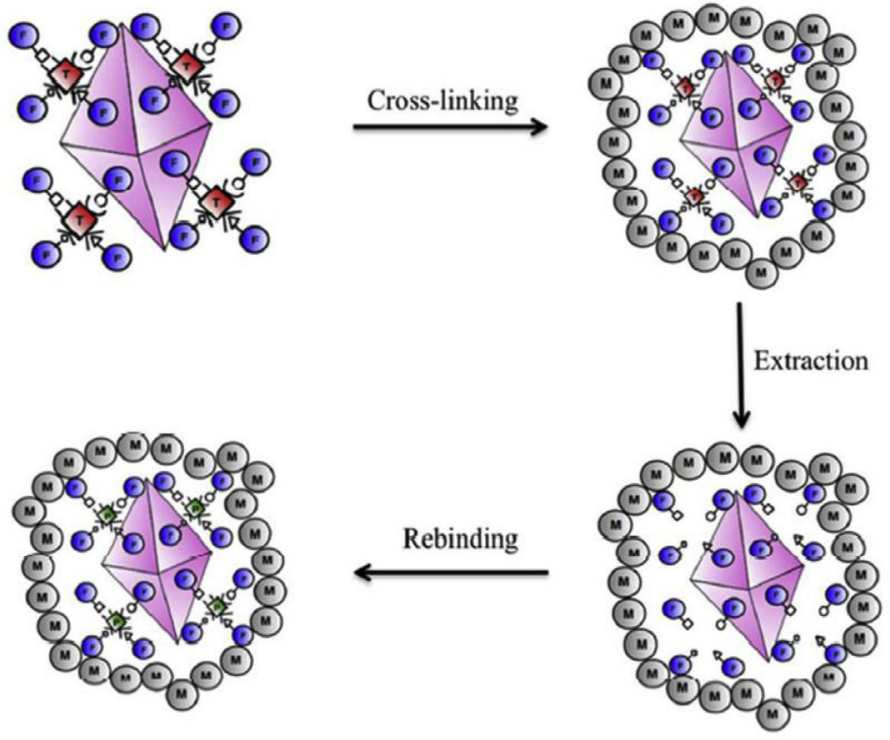

MIL-101@DMIP with Pyrraline

MIL-101@DMIP

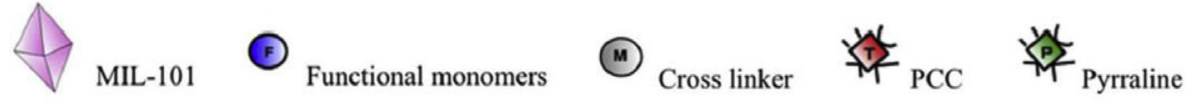

B

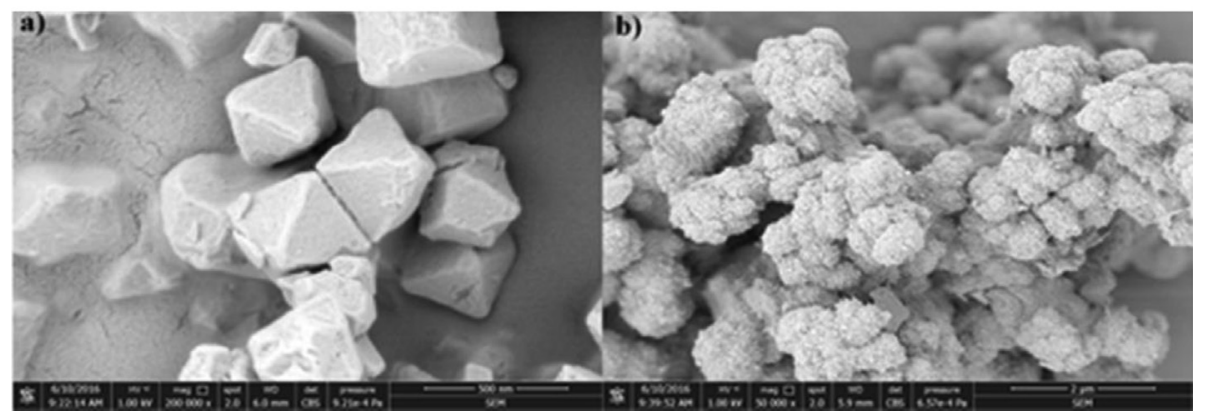

Fig. 7. (A) Schematic of the molecular imprinting process for the synthesis of MIL-101@DMIP, and (B) SEM images of (a) MIL-101, and (b) MIL-101@DMIP. Reproduced with permission [128]. Copyright $\odot 2017$ American Chemical Society.

of milk and milk powder samples by the developed MIL101@DMIP-packed SPE method followed by HPLC rendered excellent selectivity, and satisfactory efficiencies of 92.23-103.87\%.

Macromolecules imprinting is still challengeable compared with low-molecular weight compounds imprinting. The difficulty is attributed to the nature of macromolecules such as environmental instability, large molecular size, flexible conformation, and easy denaturation. Because of large and flexible structures of macromolecules, their interactions with functional monomers are unstable and not specific. Physicochemical properties of macromolecules (e.g., charge and hydrophobicity) can be significantly different, and finding appropriate functional monomers that can interact with various sites of macromolecules is difficult [129]. Furthermore, the flexibility of these molecules easily leads to their conformation change during the common polymerization conditions such as mid temperature and water miscible organic porogen. Therefore, the resulted MIPs do not show satisfactory selectivity and adsorption capacity toward target macromolecules. Compared with small organic compounds, diffusion of macromolecules into the imprinted cavities takes much more time, making the extraction time undesirably long [130,131]. In this regards, creative strategies, namely epitope imprinting [132,133], particle-based imprinting [134], micro-contact imprinting [135], and surface imprinting [136] have been developed for macromolecules imprinting. By surface imprinting, the mass transfer resistance associated with steric structure is greatly decreased. Rossetti et al. [137] introduced an assay based on surface MIPs which were synthesized relying on the surface-initiated RAFT polymerization on the surface of silica supports, targeting the proteotypic peptide of pro-gastrin releasing peptides. During the extraction process, nonspecific binding was suppressed by an extensive washing with suitable solvent following sample loading. Moreover, the desirable selectivity with a robust retention mechanism caused reproducible extraction of analyte from serum samples. However, the developed method had poor recovery yield along with a higher LOD compared with the immunoaffinity extraction method; further improvements were expected to promote the method performance.

Owing to high applicability, ease of synthesis and operation, supreme efficiency for real sample analysis, and recyclable ability of surface imprinting based MIPs, a huge number of papers have been published in this field. In Table 1, some typical applications of surface imprinting based MIPs as extraction adsorbents are listed for the determination of various analytes. 
Table 1

Summary of recent surface imprinting MIPs based sorbents for separation of different analytes.

\begin{tabular}{|c|c|c|c|c|c|c|c|c|c|c|c|}
\hline Core & $\begin{array}{l}\text { Functional } \\
\text { monomer }\end{array}$ & Cross-linker & Template & $\begin{array}{l}\text { Target } \\
\text { analyte }\end{array}$ & $\begin{array}{l}\text { Binding } \\
\text { capacity } \\
\left(\mathrm{mg} \mathrm{g}^{-1}\right)\end{array}$ & $\begin{array}{l}\text { Binding } \\
\text { kinetic } \\
(\min )\end{array}$ & $\begin{array}{l}\text { Detection } \\
\text { technique }\end{array}$ & $\begin{array}{l}\text { LOQ } \\
\left(\mu \mathrm{g} \mathrm{L}^{-1}\right)\end{array}$ & Real sample & $\begin{array}{l}\text { Recovery } \\
(\%)\end{array}$ & Ref. \\
\hline p-CMS & MAA & EGDMA & Dibenzothiophene & Dibenzothiophene & 123.7 & 150 & GC & - & Oil & $93.2-97.7$ & [220] \\
\hline $\mathrm{Fe}_{3} \mathrm{O}_{4}$ & $\begin{array}{l}\text { Dopamine } \\
\text { hydrochloride }\end{array}$ & - & Microsystin-LR & Microsystin-LR & 1.24 & 25 & HPLC-UV & - & Water & $86.0-113.0$ & [221] \\
\hline $\mathrm{Fe}_{3} \mathrm{O}_{4}$ & DES-BN & EGDMA & Quercetin & Flavonoids & - & 600 & HPLC-UV & - & $\begin{array}{l}\text { Ginkgo } \\
\text { Biloba leaves }\end{array}$ & $93.6-96.8$ & [222] \\
\hline $\mathrm{Fe}_{3} \mathrm{O}_{4}$ & - & TEOS & $\begin{array}{l}\text { Diisononyl } \\
\text { phthalate }\end{array}$ & Phthalate esters & 569.2 & - & GC-MS & $\begin{array}{l}0.0053- \\
0.0173\end{array}$ & Water & $86.7-103.1$ & [223] \\
\hline Silica gel & APTES & TEOS & Biochanin A & Biochanin A & - & - & HPLC-UV & 0.14 & Urine & $65.7-102.6$ & [224] \\
\hline MMC & MAA & EGDMA & Indomethacin & Aristolochic acid & 2952 & - & HPLC-UV & 100.0 & Rat urine & $86.7-94.3$ & [225] \\
\hline $\mathrm{Fe}_{3} \mathrm{O}_{4}$ & Chitosan & Sulfuric acid & Baclofen & Baclofen & 54.13 & - & HPLC-UV & 0.90 & Urine & $86.0-94.8$ & [226] \\
\hline $\begin{array}{l}\text { Silica } \\
\text { particles }\end{array}$ & MAA & EGDMA & Benzylparaben & Benzylparaben & - & - & HPLC-DAD & 11.2 & $\begin{array}{l}\text { cosmetics } \\
\text { and water }\end{array}$ & $99.61-101.8$ & [227] \\
\hline SBA-15 & Methyl acrylate & EGDMA & Dicyandiamide & Dicyandiamide & 1.8 & 100 & HPLC-UV & - & Bovine milk & $97.1-103.4$ & [228] \\
\hline MM & MAA & EGDMA & Triclosan & Triclosan & 1.955 & - & HPLC-UV & 0.66 & Water & $89.5-108.4$ & [229] \\
\hline $\mathrm{Fe}_{3} \mathrm{O}_{4}$ & Polydopamine & - & D-glucose & $\begin{array}{l}\text { Pulsatilla } \\
\text { Saponin Metabolites }\end{array}$ & 5.65 & 60 & UPLC-TOF-MS & - & $\begin{array}{l}\text { Metabolites } \\
\text { of botanical }\end{array}$ & - & [230] \\
\hline $\mathrm{Fe}_{3} \mathrm{O}_{4}$ & 4-VP & EGDMA & Salicylic acid & Salicylic acid & 35.4 & 30 & HPLC-UV & 300.0 & Water & $94.0-108.0$ & [231] \\
\hline
\end{tabular}

p-CMS: porous carbon microsphere; DES-BN: deep eutectic solvents based boron nitride; MMC: magnetic mesoporous carbon; MM: magnetic mesoporous.

The thickness of the MIPs layer has a major effect on the surface MIPs' performance. If the MIP layer is too thick, the defects similar to that of bulk imprinting would still arise, while too thin MIP layer would lead to the decrease of specific binding capacity and IF. In some exciting works, innovative polymerization approaches have been introduced to benefit MIP shell thickness controlling [126,132,138]. Nevertheless, controlling polymerization conditions is still challenging and need more researches.

\subsection{Hollow porous MIPS}

The attempt for improving binding capacity, complete template removal, and faster equilibrium kinetic are not limited to nano imprinting and surface imprinting. Contrary to the superior advantages of surface imprinting, sometimes, the thickness of the MIPs shell that covered solid core is more than $50 \mathrm{~nm}$. So, the template can't penetrate to the core, even although the template is adsorbed by the core and it is not specific adsorption. In other words, most of the mass of MIPs corresponding to the core is unavailable in the extraction process. This fact causes low binding capacity per unit mass of MIPs and also poor selectivity. The hybrid of MIT and porous polymers supplies novel kind of MIPs namely hollow porous MIPs (HMIPs) with favorite mass transfer and higher specific binding capacity. Universally, the preparation of HMIPs includes three steps: (i) preparing sacrificial support with competent functional groups; (ii) forming an imprinted layer on the surface of the sacrificial support; (iii) removing sacrificial support and template simultaneously that leads to generating accessible selective binding sites in the porous polymer network [139]. Polystyrene seed (PS) and silica based materials are frequently employed as versatile sacrificial supports. They are durable enough to carry MIPs layer and at the same time have considerable solubility in solvent for chemical etching [140]. By this technique, maximum binding cavities are located both at the interior and exterior of the MIPs layer. Generally, the mixture of hydrogen fluoride and alcohol is used for removing core materials and template species because of high elution strength. This step should be done carefully, because if it takes longer than the required time, binding sites of the polymer would be easily damaged. Thus, alternative solvent-free approaches like hydrothermal removing core/template is more favorable and can be considered as a green route owing to no needing hazardous solvents and no waste generation, also elimination of core/template conveniently in shorter time. However, this method can only be utilized for thermal stable polymers [141].

With this consideration, $\mathrm{Hu}$ et al. decorated monodisperse carboxylated PS with boronate-affinity MIPs by using D-glucose, 3Aminophenylboronic acid (APBA) and EGDMA as dummy template, functional monomer and cross-linker, respectively [142]. D-glucose containing a cis-diol group was covalently bonded with boronic acids of APBA in alkaline medium reversibly, and dissociated at acidic $\mathrm{pH}$. PS was dissolved in tetrahydrofuran (THF) for $3 \mathrm{~h}$ and the dummy template molecules were eluted by a mixture of methanol/ acetic acid $(8: 2, \mathrm{v} / \mathrm{v})$. The imprinting conditions were optimized to gain higher IF. Application of boronate-affinity HMIPs as solid phase sorbent for capturing daidzin, glycitin, rutin, and genistin analytes followed by HPLC/UV detection was investigated. And results indicated no matrix compound interferences during extraction and analysis processes, and the method provided satisfactory LODs from 3.5 to $10.8 \mathrm{ng} \mathrm{mL}^{-1}$.

For most of the soft sacrificing core (like PS) based HMIPs, after the formation of MIPs, dissolving/elimination and swelling of PS simultaneously occur. Because of swelling PS, the MIPs shell collapses and becomes very thin. Albeit, a slim shell of MIPs relieves binding capacity and mass-transfer, but too thin MIPs layer is fragile and breaks easily, which would destroy specific binding sites. To address this problem, Wang et al. [143] aspired new type of HMIPs for trapping estradiol from milk samples. They employed PS as core followed by introducing with vinyl- $-\mathrm{SiO}_{2}$ shell and finally MIPs shell covered the materials. Vinyl- $\mathrm{SiO}_{2}$ shell could act as a scaffold and protect MIPs from breaking. The static adsorption data had good agreement with the Langmuir model and represented homogenous binding sites coverage in MIPs shell. High IF (5.2), and fast binding kinetics (shorter than $60 \mathrm{~min}$ ) along with satisfactory recoveries for analysis of milk samples over the range of $94.8-97.0 \%$ with respective precisions of 2.5 and $2.7 \%$ were their valuable achievements.

Ji et al. used mesoporous MCM-41 as hard sacrificial support for fabrication of HMIPs for the simultaneous extraction of seven macrolide antibiotics [144]. The MCM-41 possessed superb surface areas, adjustable pore sizes, large pore volumes, and most importantly, can easily be dissolved in hydrogen fluoride solution. Besides, the degree of swelling of MCM-41 was negligible that rendered rigid and physical stable MIPs shell. The HMIPs had a higher specific surface area $\left(358.9 \mathrm{~m}^{2} \mathrm{~g}^{-1}\right)$ in contrast with solid- 
MIPs (90.5 $\left.\mathrm{m}^{2} \mathrm{~g}^{-1}\right)$ and hollow porous NIPs (HNIPs) $\left(107.0 \mathrm{~m}^{2} \mathrm{~g}^{-1}\right)$, that provided more recognition sites and influential uptake of analytes. The HMIPs based d-SPE coupled with HPLC-MS/MS method was successfully applied for selective determination of seven antibiotics from honey samples, concurrently.

HMIPs are convenient option for purification of proteins since they have a brilliant throughput with lower cost compared with antibody resins. Gómez-Arribas's team [145] employed epitope imprinting technique by using tetrapeptide DYKD and silica microspheres as template and sacrificial scaffolds, respectively for synthesis of composite MIPs. Herein, silica microspheres could boost the mass transfer of the recombinant proteins to the MIP recognition sites. In order to gain the best specific affinity toward target analyte, polymer reagents were optimized by a combinatorial approach which was designed by screening functional monomers of different polarity, as shown in Fig. 8. SDS-PAGE electrophoresis analysis revealed that the proposed HMIPs-SPE provided substantial sample cleanup and enabled the selective extraction of FLAG-derived recombinant proteins from cell lysis extracts with RSDs $\leq 15 \%$.

Unfortunately, in HMIPs-d-SPE method, owing to the low density of HMIPs, they may suspend on the top of the sample solution, so the repeated centrifugation is necessary to separate them. $\mathrm{Li}$ and co-worker tried to address this obstacle by grafting $\mathrm{Fe}_{3} \mathrm{O}_{4}$ nanoparticles on the surface of HMIPs [146]. They used MCM-48, protocatechuic acid, 4-vinylpyridine, and glycidyl methacrylate (GMA), as sacrificial support, template, functional monomer, and comonomer, respectively. After creating HMIPs, the epoxide ring of GMA was opened with $\mathrm{HClO}_{4}$ and linked to $\mathrm{Fe}_{3} \mathrm{O}_{4}$ nanoparticles to establish magnetic HMIPs (MHMIPs) and had $2.9 \mathrm{emu} \mathrm{g}^{-1}$ magnetic saturation. The MHMIPs were isolated from the sample solution by an external magnetic field with a lack of centrifugation. Although the MHMIPs had a high binding capacity $\left(37.7 \mathrm{mg} \mathrm{g}^{-1}\right)$, the IF was not very good which was possibly due to the annihilation of specific cavities in strong acid $\mathrm{HClO}_{4}$ media. Nevertheless, the method had satisfactory cleanup and no matrix compound interference during HPLC-DAD analysis of protocatechuic acid from S. aromaticum leaves.

In the last five years, well-designed HMIPs have been exploited for the extraction of various compounds from different samples such as estrogens from foods [147], caffeic acid from fruits [148], triazines from foods [149], polyphenols from drink samples [150] and glibenclamide from urine [151]. Although, creative strategies like surface imprinting and HMIPs have remarkable success for the complete template removal, still cannot assure to completely eliminate the leakage of the residual template. Therefore, dummy MIPs come to settle this issue.

\subsection{Dummy MIPs}

As noted above, different techniques have been applied for efficient removal of the template to achieve higher adsorption capacity and minimize the effect of template leakage. The leakage of template residual during the extraction process (especially in the elution step) causes tremendous analytical errors. Albeit, the concentration of the leaked template may be very low, because of high enrichment factor of SPE methods, it can lead to extreme errors on the analytical results. Therefore, the dummy template strategy for addressing the above drawbacks has always been a matter of interest. The idea of dummy imprinting first appeared in 1997, with the pioneering work of Andersson [152]. In this strategy, a similar molecule to target species is applied in terms of size, shape, and functionality with a slight difference in the synthesis process. In the next step, after removal of the dummy template, created cavities have high affinity to the target molecule due to similarity with the target template. It should be pointed out that the response of the applied analytical instrument should be different for the target and dummy template [153]. For instance, the retention time in chromatography separation should be different for analyte and dummy template with acceptable resolution. The dummy technique is used

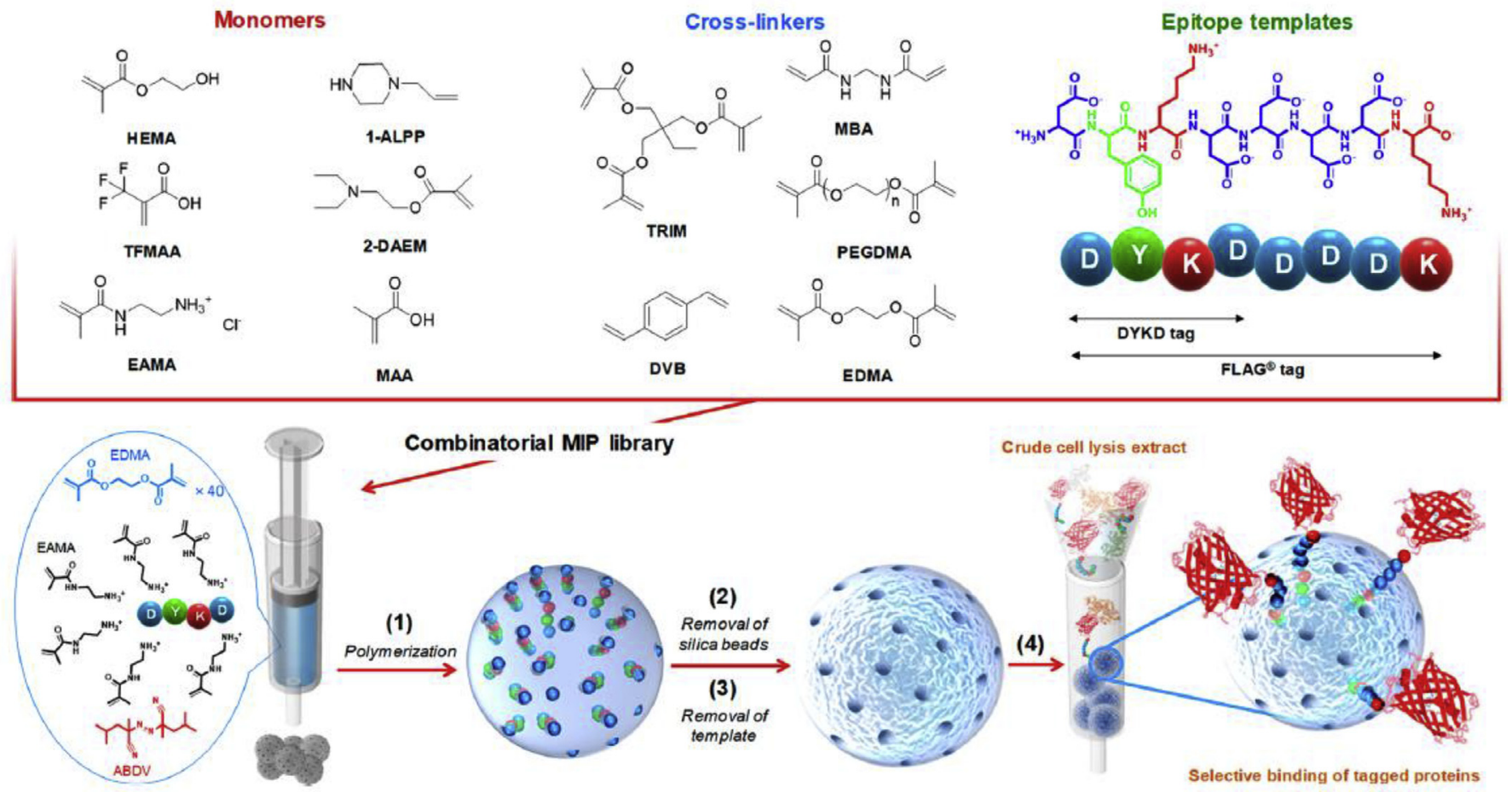

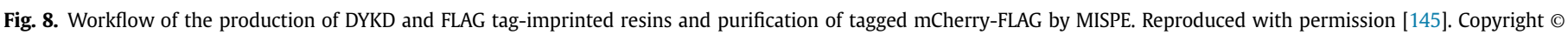
2019 American Chemical Society. 
when the target molecule is costly, not available, toxic, unstable as well as disturbance in the polymerization process. Therefore, the combination of dummy technique with other MIT has been extended promptly to achieve higher efficiency and more accurate results.

Wu and coworkers determined BPA from water and orange juice samples using dummy magnetic MIPs (DMMIPs) nanoparticles by the d-SPE method [154]. 2,2'-Bis(4-hydroxyphenyl)-hexafluoropropane was used as the analog of BPA. The synthesized MIPs showed a high binding capacity (21.755 $\mathrm{mg} \mathrm{g}^{-1}$ ) and fast binding kinetics ( $2 \mathrm{~min}$ ) owing to benefits of the surface imprinting technique on the $\mathrm{Fe}_{3} \mathrm{O}_{4}$ core. The saturation magnetization values of $\mathrm{Fe}_{3} \mathrm{O}_{4}$ and DMMIPs were 61.22 emu $\mathrm{g}^{-1}$ and 37.75 emu $\mathrm{g}^{-1}$, respectively, which advanced separation process and reduced extraction time. High enrichment factor (60), low LOD (0.3 $\mathrm{ng} \mathrm{mL} \mathrm{m}^{-1}$ ) and satisfactory recoveries (93.3-106.2\%) for both water and orange juice samples proved the high accurate analysis and applicability of the proposed method for determination of BPA.

Tan et al. prepared dummy molecularly imprinted polymers (DMIPs) on the surface of cubic like $\mathrm{Fe}_{3} \mathrm{O}_{4}$ nanoparticles for determination of aflatoxins [155]. In this study, 5,7-dimethoxycoumarin was used as the dummy template and after removal of the dummy, B1, B2, G1, and G2 aflatoxins were trapped in imprinted cavities due to the similarity in shape and functional groups. The magnetic properties of $\mathrm{Fe}_{3} \mathrm{O}_{4}$ and DMIPs were 80.0 emu g $^{-1}$ and $70.0 \mathrm{emu} \mathrm{g}^{-1}$ respectively, which showed the successful modification of MIPs shell on the surface of the magnetic core. Analysis of aflatoxins in tea-leaves and corn samples by DMIPs-d-SPE-UHPLC-MS/MS exhibited low LODs $\left(0.05 \mu \mathrm{g} \mathrm{kg}{ }^{-1}\right.$ for aflatoxins B2 and $\mathrm{G} 2$, and $0.1 \mu \mathrm{g} \mathrm{kg}^{-1}$ for aflatoxins B1 and G1), with desirable precisions.

Zhu et al. synthesized DMIPs by using theanine as a dummy template for packed SPE of eighteen amino acids in tobacco [156]. The SEM and TEM images revealed the regular spherical shape of prepared DMIPs and the thickness of DMIPs layer was approximately $40 \mathrm{~nm}$. The maximum adsorption capacity of DMIPs for eighteen amino acids was up to $1444.3 \mathrm{mg} \mathrm{g}^{-1}$, as well as, fast equilibrium kinetics (within $30 \mathrm{~min}$ ) was the remarkable advantage. The IF values of the DMIPs were achieved to be 2.86-6.9 for the eighteen amino acids, proving a clever choice of dummy molecule. The Scatchard analysis of DMIPs showed two different lines which indicated the presence of specific and non-specific binding sites in the DMIPs network. The high enrichment factors (200), the recoveries in the range of 79-104\%, and RSDs less than $7.4 \%$ demonstrated the high applicability of the proposed method for simultaneous determination of eighteen similar structured amino acids from complicated samples.
Guo et al. employed a sol-gel surface imprinting approach for synthesis of dual-dummy-template MIPs on the surface of magnetic graphene oxide (MGO) using dipropyl phthalate (DPRP) and diisononyl phthalate (DINP) as the dual-dummy templates for dSPE of phthalate esters (PAEs) from water samples and quantitatively analyzing by GC-MS [157]. Because target analytes had diverse carbon chains and chemical structures, the use of two different template molecules greatly improved the specificity of DMIPs. SEM image showed the DMIPs had a smooth appearance and almost round shape. TEM image indicated the successful modification of DMIPs layer on the surface of MGO in which the DMIPs layer had a diameter around $100 \mathrm{~nm}$ while the specific surface area of DMIPs was $304.7 \mathrm{~m}^{2} \mathrm{~g}^{-1}$. Different isotherm and kinetics models were used for investigation of the mechanism and kinetics of the DMIPs; among them, Freundlich isotherm and pseudo-second-order kinetics models were the best match with experimental results. The equilibrium time of the DMIPs was $120 \mathrm{~min}$, and the equilibrium adsorption capacities were from 2.3 to $4.8 \mathrm{mg} \mathrm{g}^{-1}$ at $298 \mathrm{~K}$ for PAEs, respectively. The LODs and LOQs of prepared DMIPs were $0.01-0.05 \mu \mathrm{g} \mathrm{L}^{-1}$ and $0.05-0.2 \mu \mathrm{g} \mathrm{L}^{-1}$, respectively, with recoveries higher than $92.9 \%$ and RSDs less than 3.8\%. In Table 2, some recent dummy MIPs used for SPE are listed.

Howbeit, worthy dummy molecules for numerous target analytes have been chosen, finding qualified dummy molecules is not simple, especially while the template is a macromolecule. Macromolecules such as proteins and cells unlike small molecules, have a bulky and twisted structure, and numerous available functional groups for the interaction with functional monomers. Hence, it is very difficult to identify the sequence and location of functional groups, and track the interaction sites of such complex templates. Therefore, this technique has no obvious progress for macromolecules template. Besides, designing, synthesis, and purification of dummy molecules are challengeable issues and should be vanquished in future works. Unluckily, sometimes dummy molecule and target analyte have the same signals in an analytical instrument or very close to each other, which causes the signal recognition to be very difficult. These weaknesses should be considered and overcome for extending the dummy imprinting strategy.

It has been frequently reported that the most important superiority of MIPs compared with ordinary sorbents is their intrinsic selectivity. For improving the selectivity and anti-interference ability of MIPs, several ways are suggested: designing and synthesizing new functional monomers with higher affinity, employing computational tool to evaluate the monomer and template interactions for finding optimum functional monomer, investigating

Table 2

Summary of different types of dummy MIPs sorbents for separation of different analytes.

\begin{tabular}{|c|c|c|c|c|c|c|c|c|c|}
\hline Dummy molecule & Target analyte & SPE mode & $\begin{array}{l}\text { Detect } \\
\text { technique }\end{array}$ & IF & $\begin{array}{l}\text { Adsorption } \\
\text { capacity } \\
\left(\mathrm{mg} \mathrm{g}^{-1}\right)\end{array}$ & $\operatorname{LOQ}\left(\mu \mathrm{g} \mathrm{L}^{-1}\right)$ & Real sample & Recovery (\%) & Ref. \\
\hline Miconazole & Climbazole & SPE & HPLC-DAD & 7.0 & 7.506 & - & Water & $82.3-96.2$ & [232] \\
\hline 4,4-HDPE & $\begin{array}{l}\text { Polybrominated } \\
\text { diphenyl ethers }\end{array}$ & SPE & GC-MS & $1.1-4.0$ & 0.00260 & - & Environmental & $43.0-92.0$ & [233] \\
\hline THPE & Bisphenols & SPE & HPLC-MS/MS & - & - & $0.0000007-0.0163$ & $\begin{array}{l}\text { Sewage and } \\
\text { sludge }\end{array}$ & $43.6-101.0$ & [234] \\
\hline $\begin{array}{l}\text { 2-phenylpropionic } \\
\text { acid }\end{array}$ & NSAIDs & d-SPE & HPLC-UV & $1.4-3.3$ & $6.5-9.7$ & $0.00093-0.00220$ & Water & $72.43-113.99$ & [235] \\
\hline Indole-3-acetic acid & Domoic acid & SPE & HPLC-DAD & - & - & 30.0 & Seawater & $91.5-94.8$ & [236] \\
\hline BPAF & BPA & SPE & HPLC-UV & - & 2.416 & 1.0 & Water & $97.3-106.0$ & [237] \\
\hline Bisphenol B & $\mathrm{BPA}$ & d-SPE & HPLC-UV & - & - & 0.01 & Water & $65.56-88.84$ & [238] \\
\hline DCE & Imidazole fungicides & SPE & HPLC-DAD & $10.7-10.9$ & - & - & River water & $84.2-95.0$ & [239] \\
\hline Nonylphenol & Vanillin & SPME & HPLC-UV & - & - & 1.5 & Milk powder & $83.0-110.0$ & [240] \\
\hline
\end{tabular}

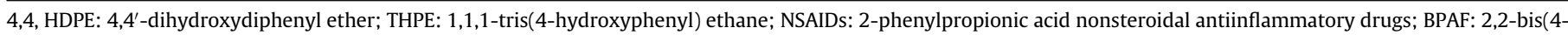
hydroxyphenyl) hexafluoropropane; DCE: alpha-(2,4-dichlorophenyl)-1H-imidazole-1-ethanol. 
porogen type's effect on the selectivity, and using binary or a mixture of functional monomers for MIPs preparation.

\subsection{Multi-functional monomer MIPs}

It is well known that the selection of functional monomer is a vital step in MIPs preparation since the appropriate interaction between the target template and functional monomer not only can reduce the leakage of target molecules during the extraction process, but also can increase the stability of prepared MIPs [158,159]. The interaction of the target molecule and functional monomer is conducted based on the covalent and non-covalent interactions. In the covalent approach, the functional monomer only interacts with one side of the template molecule through covalent interaction with stoichiometry ratio, which limits the number of effective interactions and adsorption capacity. In contrast, in the non-covalent approach, functional monomer interacts with a template molecule with different sites through non-covalent interactions such as Van der Waals forces, hydrogen bonding, and acid-base Lewis. On the other hand, in the non-covalent method, an excess molar ratio of a functional monomer is used to make sure that functional monomer and template completely interact and form lasting pre-complex. The specific adsorption of MIPs is done based on the size, shape and functional groups of the template. Because changing in the size and shape of template is illogical and infeasible, in order to have more interactions between template: functional monomer, it can be possible to enhance the selectivity of MIPs by increasing the interactions among template and functional groups of monomers [160]. However, customary MIPs suffer from poor selectivity and binding capacity. To further promote MIPs' efficiency, selectivity and stability, designing of MIPs with bi and/or multi-functional monomer via varied interactions has attracted enormous attention.

Generally, the selection of optimum functional monomers is accomplished by trial and error approach; however, it is laborious and time consuming. Computational modeling is a more preferable method for investigation of bonds strength and dominant possible interactions between a template and functional monomer. Accordingly, in this way, the design of MIPs is screened before the synthesis of polymers, and ultimately MIPs are synthesized without generation of massive wastes in agreement with green chemistry concepts [161,162]. Gaussian software is regarded as the most widely used quantitative software for semi-empirical calculation. For example, Gaussian software ab initio calculation was employed for designing uniform spherical MIPs particles for chenodeoxycholic acid by suspension polymerization [163]. Using hydrophilic cross-linker namely polyethylene glycol (PEG) diacrylate 200 enhanced the hydrophilicity of the polymer surface and increased the MIPs performance in aqueous media. This work is a first report splitting method in MIT in which the polymer network was modeled by computational modeling. Computational modeling is an effective tool for prediction of stability of macromolecule template-functional monomer complex as well. In the other work, the possible binding interactions between myoglobin protein template and five different acrylamide-based functional monomers was evaluated by using computational technique [164]. SiteMap and MM-GBSA methods were selected for predication of protein binding sites and free energies of monomers at each site, respectively. Also, far-UV circular dichroism spectroscopy was used for determination of monomer effects on myoglobin secondary structure. The results revealed that monomers with hydroxyl functional groups had generally stronger binding free energy $\left(\Delta \mathrm{G}_{\mathrm{PL}}\right)$ values than those without the hydroxyl groups, and experimental validation was in line with the predictions.

Unfortunately, the problem of heterogeneity of binding sites in non-covalent MIPs cannot be thoroughly solved by using multi- functional monomer strategy. Therefore, the adsorption mechanism of non-covalent MIPs commonly follows the Freundlich isotherm model [165,166]. The Freundlich isotherm model can provide the information of the binding site homogeneity for most non-covalent MIPs, where its heterogeneity index varies from 0 to 1 , with 1 being homogeneous and values approaching 0 being increasingly heterogeneous. In addition, it has also been wellestablished that the MIP particles have more heterogeneous binding sites than the NIPs in most cases [167]. Oriented strategy can remarkably improve the homogeneity of binding sites [168,169]. Boronate affinity based MIPs are the famous materials in this category [170]. In some cases, supreme MIPs have been designed containing both covalent and non-covalent interactions between monomers and template to reach higher selectivity $[171,172]$. Hereupon, some researchers focus on the synthesis of multifunctional monomer MIPs, with the combined use of other imprinting strategies.

Duan and colleagues prepared water-compatible MIPs by using surface imprinting and dual functional monomer imprinting strategies for the determination of BPA from aqueous solution [173]. GO was used as support, and 2-acrylamido-2-methylpropanesulfonic acid (AMPS) and styrene (St) were used as dual functional monomers. AMPS and St introduced hydrogen-bonding and $\pi-\pi$ interactions, which enhanced the adsorption capacity of proposed MIPs. The highest adsorption capacity of $85.7 \mathrm{mg} \mathrm{g}^{-1}$ was obtained in the molar ratio (AMPS: St) of 2.5:2.5. Also, the adsorption kinetics study revealed that the equilibrium time for AMPS-St/MIPs was at $60 \mathrm{~min}$. The high selectivity and ability of the MIPs for recognition of BPA from analog compounds were proved as well.

Yuan et al. synthesized a magnetic core-shell MIPs by the RAFT polymerization using bifunctional monomers system for extraction and HPLC-UV determination of BPA from milk [174]. As seen in Fig. 9, 4-vinylpyridine (4-VP) and $\beta$-cyclodextrin ( $\beta$-CD) were chosen as two functional monomers. 4-VP as one of the common functional monomers can provide hydrogen-bonding sites. And, $\beta$ $C D$ has a superior number of secondary hydroxyl groups and their cavities are compatible with the target molecules in terms of size, shape and functional groups. Therefore, the combination of 4-VP and $\beta-C D$ was a promising option for enhancement of the applicability of MIPs. The TEM image indicated uniform spherical morphology of prepared magnetic MIPs with a diameter of $320 \mathrm{~nm}$. The magnetization value of the magnetic MIPs was $35.18 \mathrm{emu}^{-1}$, lower than that of $\mathrm{Fe}_{3} \mathrm{O}_{4} @ \mathrm{SiO}_{2}\left(74.65 \mathrm{emu} \mathrm{g}^{-1}\right.$ ), which confirmed the successful covering of MIPs layer onto the $\mathrm{Fe}_{3} \mathrm{O}_{4} @ \mathrm{SiO}_{2}$ surface. The adsorption capacities of $\mathrm{Fe}_{3} \mathrm{O}_{4} @ \mathrm{SiO}_{2}, \beta-\mathrm{CD}, \mathrm{Fe}_{3} \mathrm{O}_{4}$-NIPs, and $\mathrm{Fe}_{3} \mathrm{O}_{4}$-MIPs for BPA were found to be $0.90,4.21,4.65$ and $17.98 \mathrm{mg} \mathrm{g}^{-1}$, respectively, clearly proving the positive effect of bifunctional system on the adsorption capacity. The obtained recoveries were between 97.23 and $99.21 \%$ with RSDs within 2.9-3.8\%, which showed the highly accurate determination of BPA from complicated milk samples.

Arabi et al. synthesized hydrophilic nano-sized dummy MIPs for PT-SPE of prednisolone from urine sample [175]. In this work, 3aminopropyltrimethoxysilane (APTMS) and aluminum ion $\left(\mathrm{Al}^{3+}\right)$ were utilized as two functional monomers, and they both introduced hydrogen-bonding and Lewis acid sites in the silica matrix for metal coordinative interactions with the prednisolone, respectively. Compared to traditional SPE methods, the proposed MIPsPT-SPE approach was cost-effective, fast, and easy to handle with consumption of low volume of toxic organic solvents. To overcome the prednisolone leakage, dummy and dual functional monomer strategies were combined. In the extraction process, the elution step was done under ultrasound irradiation by immersing the pipette-tip column in an ultrasonic bath which reduced desorption time and eluent solvent consumption. The high adsorption capacity 


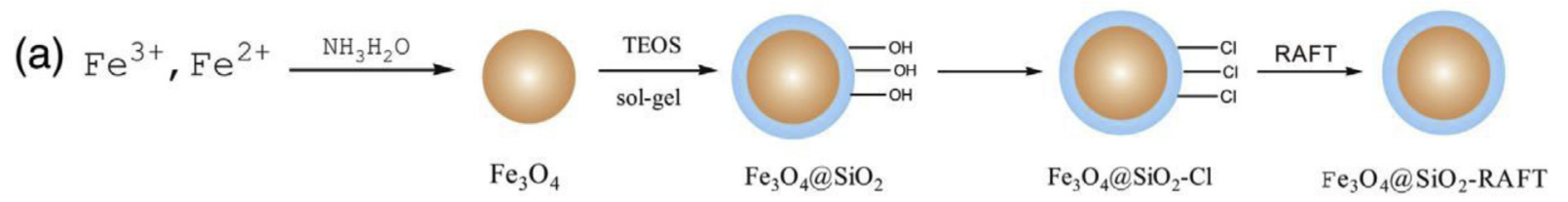

(b)

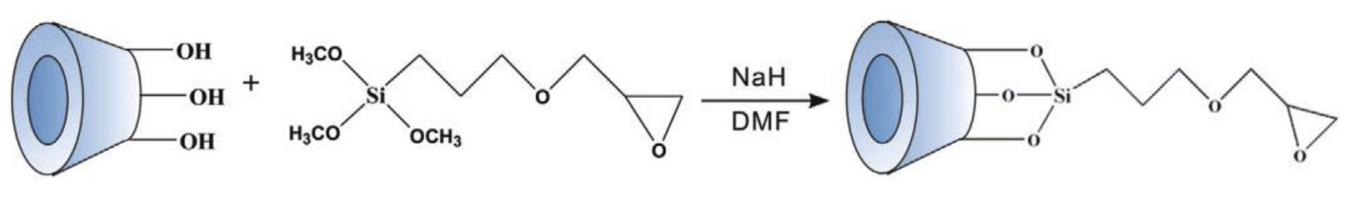

(c)

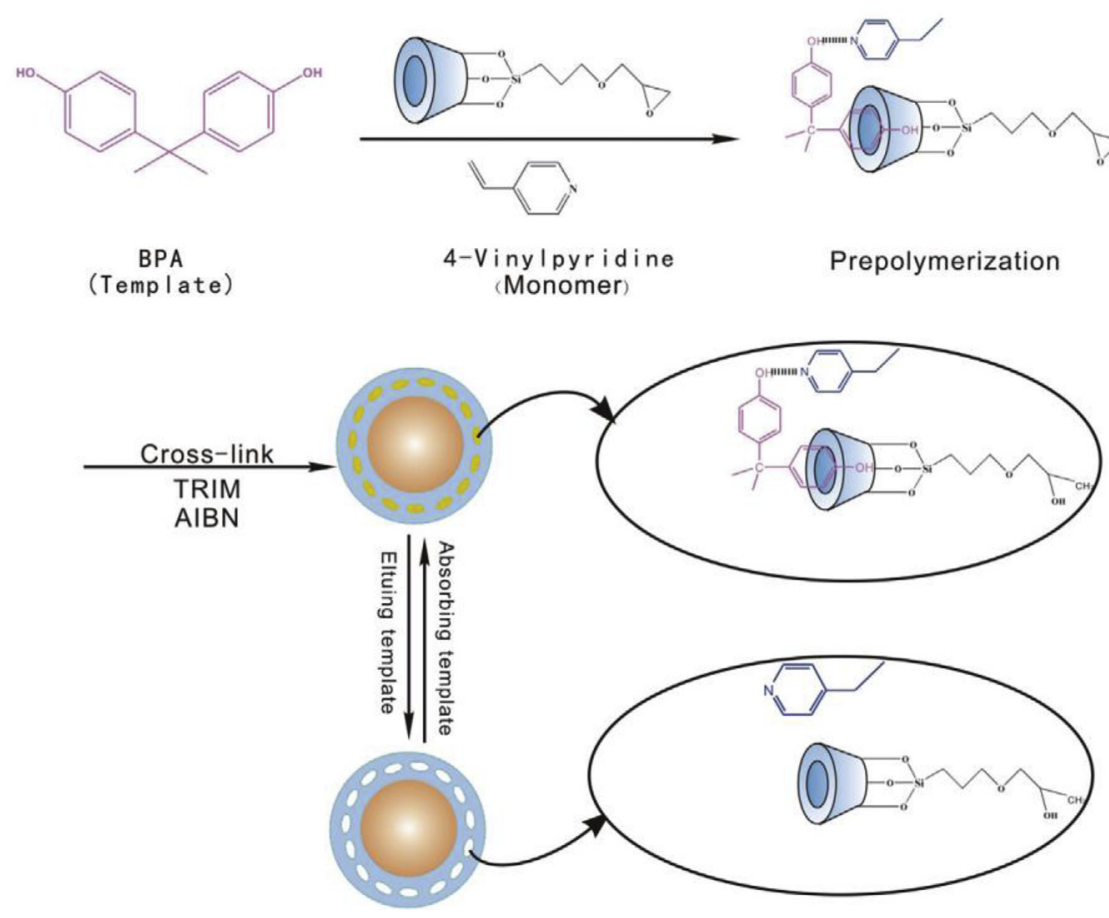

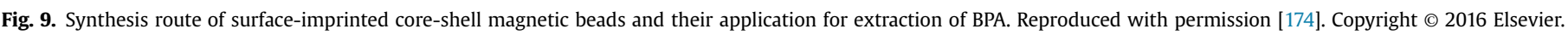

of prepared MIP ( $42 \mathrm{mg} \mathrm{g}^{-1}$ ) can be attributed to the high interaction of prednisolone and dual functional monomers. The proposed method showed a wide linear range of $0.22-220 \mu \mathrm{g} \mathrm{L}^{-1}$ and satisfactory recoveries of $89.0-96.1 \%$ with RSD values lower than $9.0 \%$.

To gain ideal multi-functional monomer MIPs with satisfactory selectivity, the molar ratio of functional monomers and cross-linker should be optimized attentively. Several major technical points of this multi-functional monomer imprinting strategy should be further explored, such as, how to select appropriate dual/multiple functional monomers and wisely perform the combined use, how to devise and synthesize new functional monomers, how to effectively use their synergistic effects, and provide which functional monomers to overcome the disability for template molecules with lack of functional group.

\subsection{Multi-template MIPS}

From past to now, the demand for monitoring and quantitative determination of chemicals in different samples has been dramatically increased. Simultaneous analysis of a group of compounds saves time and economizes the cost. On the other hand, the probability of occurring errors in sequential pretreatments for each analyte individually is increased. By these reasons, the analysis of a series of analytes is preferred to single analyte detection undoubtedly. In the most of MIPs, a single template molecule is utilized to construct the selective cavities of the MIPs. These kinds of MIPs may be feasible for the uptake of extremely similar a group of analytes, but they are unusable for extraction of dissimilar analytes in terms of size, shape, and functionality. Formerly, for enhancing the practical value of MIPs in simultaneous analysis, two or more 
individual MIPs were physically mixed. However, in this method, several kinds of MIPs should be synthesized, which demands too many chemicals and much time, and consummate homogenization of the polymers is also a great challenge. In this regards, the multitemplate strategy has been proposed, defined as using two or more templates and supplying manifold sorts of recognition sites in one MIPs format [176,177]. By employing multi-template MIPs (mtMIPs) as sorbent, recognition and extraction of a group of possible contaminants can be carried out synchronously [178].

Lu et al. used six phenolic compounds as the templates including phenol, 4-chlorophenol (4-CP), 2,4,6-trichlorophenol (2,4,6-TCP), 2,4-dichlorophenol (2,4-DCP), 2-chlorophenol (2-CP) and 2,6-dichlorophenol (2,6-DCP) for the fabrication of mt-MIPs by precipitation polymerization and subsequently employed it as the sorbent in packed SPE [179]. The mt-MIPs possessed great adsorption capacities in the range of $130-400 \mathrm{mg} \mathrm{g}^{-1}$. The IF values of six template compounds were obtained above 2.02 , but only 1.38 for 3-CP, 1.23 for PCMC, 1.16 for 4-NP and 1.23 for 2-NP, suggesting the good imprinting effect. Under the optimized conditions, the mtMIPs-SPE coupled with capillary electrophoresis method was applied to different water samples; $82.13-105.63 \%$ recoveries and $1.68-6.96 \%$ RSDs confirmed notable potency of mt-MIPs for the simultaneous analysis of target pollutants within a short time.

Sun and coworkers fabricated mt-MIPs by using SBA-15 as solid support, the components of ginsenoside Rb1 (Rb1), ginsenoside Rg1 (Rg1) and notoginsenoside R1 (R1) as templates, acrylamide as a functional monomer and EGDMA as cross-linker [180]. According to chemical structure and functional groups of templates, the ratio of Rb1 to Rg1 to R1 of 44:43:13 in ethanol porogen was selected for preparation of mt-MIPs. Adsorption results revealed the highest adsorption capacities for mt-MIPs and NIPs were 123.11 and $58.41 \mu \mathrm{mol} \mathrm{g}{ }^{-1}$, respectively, with IF 2.11 which was a witness for the successful creation of three types of specific cavities in the mtMIPs network. The mt-MIPs had excellent stability and could be recycled for six times. Despite the complexity of plasma samples, after mt-MIPs-SPE, most of the impurities were removed and three analytes' peaks appeared significantly within $36 \mathrm{~min}$ in HPLC analysis.

B-family vitamins are biologically important compounds that control human normal health. Simultaneous selective extraction of B-vitamins is difficult due to high water solubility and disparate chemical structures. Our team, prepared hydrophilic multitemplate molecularly imprinted biopolymers (mt-MIBP) by using riboflavin $\left(B_{2}\right)$, nicotinamide $\left(B_{3}\right)$ and pyridoxine $\left(B_{6}\right)$ as templates, and green and natural bio-substances (chitosan and glucose) [181]. First, glucose was carbonized by a hydrothermal route to obtain carbon spheres (CS) and acted as a porous core, and then chitosan network encompassed three templates to cover CS and finally mtMIBP core-shell materials were attained, as schematically shown in Fig. 10. Interestingly, all synthesis steps occurred in aqueous media without the consumption of organic solvent. The application of mtMIBP in d-SPE for extraction of B-vitamins followed by HPLC-UV determination was investigated, and the influences of the individual variables and their interactions on d-SPE efficiency were optimized by experimental design methodology. Because the target analytes had a different structures, we expected that the optimal detection wavelengths for them may be different. Hence, the wavelengths of UV were examined in the range of $265-290 \mathrm{~nm}$ with $1 \mathrm{~nm}$ intervals. Evaluating the chromatograms suggested that the maximum peak areas were achieved at the wavelengths of 270 , 274 , and $284 \mathrm{~nm}$, for $\mathrm{B}_{2}, \mathrm{~B}_{3}$, and $\mathrm{B}_{6}$ respectively. Finally, the mtMIBP-d-SPE-HPLC method was successfully applied for clean-up and extraction of B-family vitamins from orange juice samples.

Generally, the adsorption capacity of mt-MIPs for each analyte is lower than individual MIPs. This phenomenon is related to the creation of several types of specific cavities in a MIPs network, while in single template MIPs, only one type of specific binding site exists. In other words, the number of specific cavities for specific template in the individual MIPs is much more than that in the mtMIPs. Hence, increasing adsorption capacity of mt-MIPs can promote the efficiency of these materials. Heterogeneity of binding sites is the other fault of mt-MIPs and needs to be inspected for further works.

All mentioned above, MIPs recognition performance and selectivity are often suppressed in pure aqueous media compared with organic solvents. Hence, researchers purpose to synthesize upgraded MIPs to solve this problem. In this regard, water compatible MIPs have been introduced and developed.

\subsection{Water compatible MIPs}

Unlike selective biological receptors such as enzymes and antibodies, ordinary MIPs usually suffer from the lack of prominent recognition ability in aqueous solutions. This defect is negligible in the peptide or protein imprinting owing to intrinsically water compatibility of these materials [182]. However, the affinity and specific recognition ability of MIPs toward small organic molecules in aqueous media are inappropriate. This phenomenon can be explained by a high degree of swelling in an aqueous solvent which causes resizing and deformation of imprinted cavities and hinders the entering of target analytes. That is to say, maximum selectivity and the rebinding ability of MIPs occur while MIPs are exposed to the solvent used for their preparation, which is called "porogen memory" [183]. Formation of sustainable template-functional monomer complexes in appropriate polar organic solvents is the essential step of MIPs construction. When MIPs are exposed to water-rich solutions, the interaction between template and monomer especially hydrogen bond will be destroyed, since water molecules could act as both acceptors and donors of hydrogen binding and compete forcefully with template molecules, and eventually the specific recognition performances of MIPs will be decreased [184]. Note that this matter is highlighted in MIPs-SPE methods, because the selectivity of MIPs sorely depends on the nature of the sample and should be optimized by the choice of solvents in the extraction procedure. Most samples are aqueous media, so the target analytes should be separated from water solutions, and great endeavors have been assigned to designing prominent MIPs that can be directly operated for selective capturing analytes from this media. Undoubtedly, the first report of water-compatible MIPs can be attributed to Mosbach's group in 1993 , because they emphasized on recognition in aqueous systems [185].

Recently, extensive researches have been performed to develop water-compatible MIPs and the ideas can be divided as (i) fabrication of MIPs in the buffer and aqueous systems [186], (ii) using hydrophilic MIPs' precursors, especially functional monomers and functional co-monomers such as 2-hydroxyethyl methacrylate, $\beta$ cyclodextrin, allyl glucose, chitosan, gelatin, sodium alginate and etc. [187], (iii) post-imprinting approaches viz. modification surface of MIPs by hydrophilic polymer layers or decoration of MIPs surface by hydrophilic functional groups (iv) preparation of MIPs by oil-in water Pickering emulsions polymerization, (v) stimuli-responsive MIPs, and (vi) controlled/“living” radical precipitation polymerization using hydrophilic macromolecular chain-transfer agents. Among these techniques, using aqueous systems as porogen and hydrophilic functional monomers are more facile and straightforward; hence, both have been employed more than others recently [188-190].

Sellergren and his group as a pioneer in this area have carried out a series of distinguished researches in the synthesis of water 


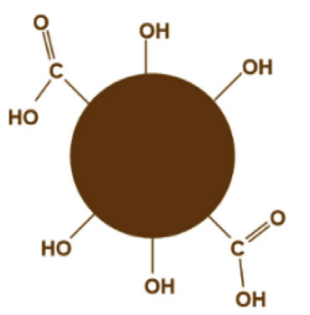

Carbon sphere (CS)

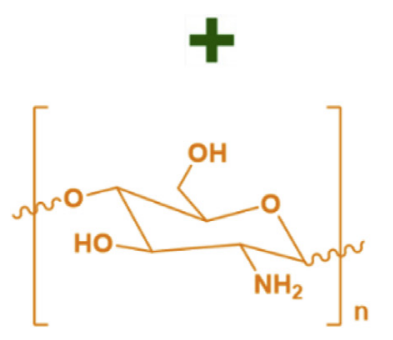

Chitosan<smiles>OC[C@H]1O[C@H](O)[C@@H](O)[C@H](O)[C@H]1O</smiles>

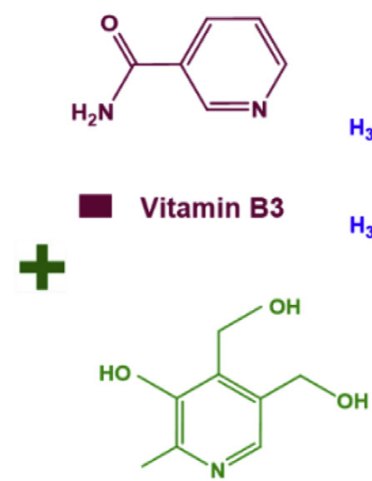

$\triangle$ Vitamin B6<smiles>Cc1cc2c(cc1C)N(C[C@H](O)[C@H](O)[C@H](O)CO)C1NC(=O)NC(=O)C1=N2</smiles>

Vitamin B2

mt-MIBP

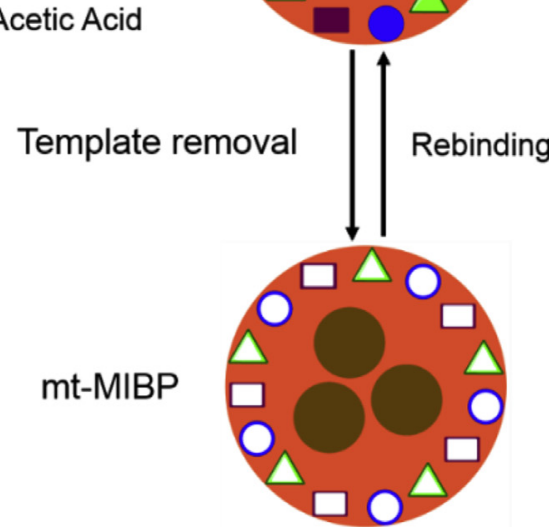

Fig. 10. Schematic of mt-MIBP preparation. Reproduced with permission [181]. Copyright @ 2018 American Chemical Society.

compatible MIPs. For example, they fabricated a water-compatible stochiometrically imprinted polymer for extraction of fluoroquinolones from aqueous samples [191]. Stoichiometric imprinting is to design variety of non-covalent MIPs, in which host monomers providing strong interactions with the templates' functional groups. This leads to enhancing the stability of the monomer: template complexes which, in turn, translates into a high-yield generation of sites of better definition in the final polymer. Therefore, the stoichiometric imprinting increase the applicability of prepared MIPs in water based media. In this work [191], MIPs were synthesized by using enrofloxacin as the template and a stoichiometric quantity of urea-based functional monomer to target the single oxyanionic moieties in the template molecule. The urea-based functional monomer namely 1-(4-vinylphenyl)-3-(3,5bis(trifluoromethyl)phenyl) had stronger interactions with the template compared with routine functional monomers. The main point about prepared MIPs was its applicability for more than 80 assays without losing their pre-concentration ability, which is promising for on-line pre-concentration formats. The results also confirmed the high anti-interface ability of the MIPs.

In the other work of Sellergren's group, water compatible MIPs were designed and used as receptor of water soluble $B_{2}$ vitamin [192]. The proposed MIPs were synthesized using riboflavin tetraacetate as a template and pentaerythritol triacrylate (PETA) as a hydrophilic cross-linking monomer. In spite of successful imprinting, nonspecific adsorption of riboflavine was also observed. Hence, to improve the selectivity of water compatible MIPs, an alkaline post-treatment was performed. The preferred approach consisted of a controlled base hydrolysis of pendent unreacted acrylate groups, using hydroxides with differently sized counter ions as reagents. By this method, the swelling of MIPs and subsequently the loss of the imprinting effect were decreased. Owing to upgrade the performance of the MIPs by post-imprinting, riboflavine was selectively separated from milk, beer and a multivitamin mixture.

Zhang and his group also have a prominent role for advancement of water compatible MIPs. They introduced a one-pot and efficient approach for synthesis of pure-water-compatible and narrowly dispersed MIP microspheres with surface-grafted hydrophilic polymer brushes by facile RAFT precipitation polymerization mediated by hydrophilic macromolecular chain-transfer agents [193]. The presence of hydrophilic polymer brushes on the surface of MIP microspheres significantly improved their surface hydrophilicity and dramatically reduced hydrophobic interactions toward template molecules in pure aqueous media, thus leading to the enhancement of water compatibility. Because the hydrophilic macromolecular chain-transfer agents are easily available and RAFT polymerization methods are versatile, the approach is highly applicable for the design of hydrophilic and water-compatible MIPs. Different chain-transfer agents with various chemical structures and molecular weights were prepared and used as the RAFT agents. Results confirmed that the chain-transfer agents can control the shape of MIPs and also change the size of materials.

In the other work of the same group, for the first time, narrowly dispersed highly cross-linked MIP nanoparticles with surfacegrafted hydrophilic polymer brushes in a facile one-pot approach was prepared via RAFT polymerization mediated by using hydrophilic macromolecular chain-transfer agents [194]. By the proposed approach, not only the hydrophilicity of synthesized MIPs was enhanced, but also chain-transfer agents acted as a protective layer to avoid accumulation of proteins in the biological samples on the surface of nanoparticles and blocking the imprinted sites. On the other hand, the RAFT method provided a condition to control the 
thickness of MIPs and introduce narrowly dispersed hydrophilic MIP nanoparticles. Finally, excellent specific molecular-recognition ability of the MIPs was proved, in real aqueous solutions including river water and biological samples (both diluted and undiluted milk and bovine serum).

Zhao et al. prepared water compatible MIPs based on RAFT precipitation polymerization for the simultaneous extraction of six sulfonamides from different complex samples like seafood, egg, milk and tissue followed by HPLC-MS/MS determination [195]. Interestingly, the whole pretreatment steps were carried out without organic solvent consumption. According to the competitive adsorption test results, the adsorption capacities of MIPs were strikingly lower for interference compounds such as enrofloxacin, penicillin $\mathrm{G}$, ampicillin and 2-NP-AHD, that is owing to the privilege of specific binding sites in MIPs network. By using MIP-SPE, due to the influential clean-up ability of MIPs, the matrix effects for all the six sulfonamides were negligible, ranging from $85.30 \%$ to $99.94 \%$ in the ten samples. However, for commercial-SPE columns, the matrix effects were much higher and interference compounds were coextracted with target analytes. The HPLC-MS/MS analysis was carried out in gradient mode and all analytes were determined in the retention time of $2-8 \mathrm{~min}$. The linearity of the proposed method for all analytes was achieved in the wide range of $0.001-2.5 \mathrm{mg} \mathrm{L}^{-1}$, with satisfactory precisions of lower than $4.68 \%$ for ten repeated analysis.

$\mathrm{Wu}$ and co-workers successfully prepared water-compatible, temperature, and magnetic dual-responsive MIPs (WC-TMMIPs) by RAFT precipitation polymerization [196]. The versatile WCTMMIPs possessed noteworthy benefits, namely fast adsorption kinetics, large binding capacity, desired selectivity, and excellent magnetization saturation that made WC-TMMIPs as a qualified sorbent for selective extraction of BPA from seawater samples. Fig. 11 schematically illustrates the synthesis procedure of WCTMMIPs, and their applications for the reversible adsorption and release of BPA by temperature controlling around SPE column and also MSPE (namely d-SPE) method (A), as well as possible molecular recognition mechanism regulated by temperatures (B). Results showed that the d-SPE was faster than packed-SPE while packedSPE offered more impressive enrichment. The WC-TMMIPs based SPE coupled with HPLC-UV analysis for BPA exhibited worthy linearity in the range of $0.1-14.5 \mu \mathrm{M}$ and $1.3-125 \mathrm{nM}$, with low LODs of $0.02 \mu \mathrm{M}$ and $0.18 \mathrm{nM}$ for packed-SPE and d-SPE, respectively. By reviewing literatures in the field of water compatible MIPs, it can be concluded that RAFT precipitation polymerization is a facile and impressive technique for fabrication of these kinds of materials [197-200].

Wang et al. fabricated water compatible molecularly imprinted resins (MIRs) in a water solution of the triblock copolymer as porogen to dredge the network structure of MIRs [201]. The resin was synthesized by hydrophilic reagents including melamine, urea, and formaldehyde. Because a high abundance of amino groups (secondary and tertiary) could induce a positive charge in a certain $\mathrm{pH}$ range and produce ion exchange interaction with negativecharge carrier molecules, the ion exchange interaction is stronger than hydrogen bonding and the by-effect of water is minimized. Also, for the elimination of template leakage, dummy molecule ( $\mathrm{N}$ (1-naphthyl) ethylenediamine dihydrochloride) was used in synthesis procedure and the MIRs were utilized in PT-SPE method for extraction of four plant growth regulators compounds followed by determination via HPLC-UV. To investigate the MIR performance in coconut juice media, loss ratios of analytes (defined as the peak area of analytes in effluent, split up on the peak area of analytes in standard water solution $\times 100$ ) by the MIRs and various commercial sorbents including Florisil, silica, $\mathrm{N}-\mathrm{Al}_{2} \mathrm{O}_{3}, \mathrm{NH}_{2}$-silica, $\mathrm{C}_{18}, \mathrm{SCX}$, HLB, and MCX were assessed and compared; high applicability of
MIRs was certified. Supreme molecular recognition in aqueous matrices, extraction procedure in the line of green chemistry, and validated accurate method are the highlights of this work. In the other two researches by the same team, they fabricated different kinds of hydrophilic MIRs via a simple and green protocol for capturing plant hormones [202,203] and bovine serum albumin $[202,203]$. Both resins showed high adsorption capacities, fast mass transfer rates, and specific selectivity towards target analytes in aqueous solvent.

It is no doubt that by developing water compatible MIPs, promising synthetic receptors are provided at the service of such applications as clinical diagnostics, food and environmental analysis. However, for broad practical applications, two imperfections still accompany with water compatible MIPs. One is the heterogeneity of binding sites which induces the cross-selectivity. The other is nonspecific binding towards the template molecules in aqueous media. It is indispensable to ameliorate the surface hydrophilicity of the MIPs by hydrophilic brush/layer and enhance the specific binding in both aqueous and organic or organic solvent-rich media.

As we discussed above, pleasantly the incompatibility of MIPs in aqueous samples can somewhat be overcome by alternative water compatible MIPs. A similar problem also exists for the analysis of small organic molecules in complex samples containing hydrophobic interferences [204,205]. In the following, we discuss this limitation.

\subsection{Restricted access material combined MIPs (RAM-MIPs)}

Biological macromolecules such as proteins and lipids in biological samples could be strongly adsorbed on the surface of MIPs and prevent the mass-transfer of analytes from the sample solution to recognition cavities. This impediment can be addressed by using restricted access materials (RAMs) for the determination analytes of interest from complicated biological samples. The first report of RAM-MIPs was attributed to Haginaka's team in 1999. They synthesized selective MIPs with a hydrophilic external layer for enantio separation of (S)-naproxen from serum samples [206]. RAMs act based on the size exclusion in which small molecules (target templates) are allowed to enter to predefined specific cavities of MIPs, while macromolecules are excluded to access the inner functional groups and cavities of MIPs [207]. Considering this problem, Zuo and coworkers synthesized novel RAM-MIPs for extraction of heptachlor, endosulfan and their metabolite residues in pork followed by determination via GC with an electron capture detector (GC-ECD) [208]. The RAM-MIPs were prepared by precipitation polymerization and showed high affinity for adsorption of endosulfan and effectively prevented the adsorption of macromolecules on the surface of RAM-MIPs. In the other work published by Zuo' group, they determined the same analytes in pork by another technique. Since the matrix of pork is complex, it needs an influential sample pretreatment method before instrumental analysis. In this regard, they employed gel permeation chromatography (GPC) and florisil SPE cartridge for clean-up, which endured disadvantages such as several boring steps, waste generation, and expensive instrument [209]. The obtained LODs were in the range of $0.002-0.003 \mathrm{mg} \mathrm{kg}^{-1}$, with RSDs of $4.0-11.3 \%$. Excitingly, their synthesized RAM-MIPs were a competent alternative for enrichment and clean-up of eight compounds in pork without using GPC and florisil SPE cartridge. The obtained LODs were $0.001-0.002 \mathrm{mg} \mathrm{kg}^{-1}$ and recoveries were $51.17-107.73 \%$ with RSDs of $1.4-15.9 \%$, which certified the successful application of the synthesized RAM-MIPs for determination of target analytes from complicated pork sample. The hydrophilicity of MIPs and RAMMIPs was investigated separately using water dispersion and protein binding experiments. Results revealed that RAM-MIP had 

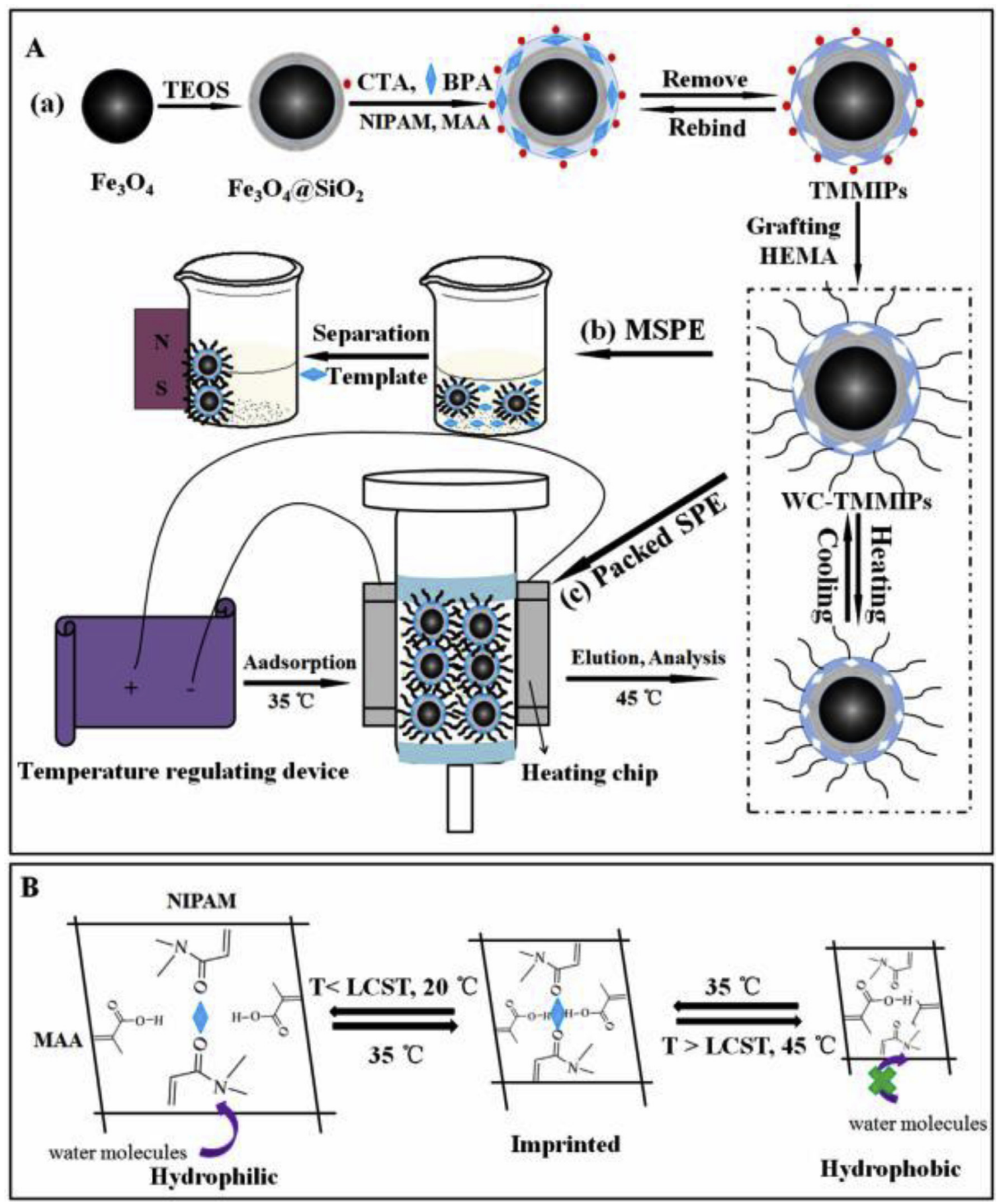

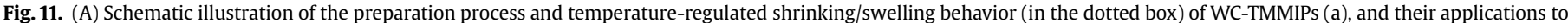

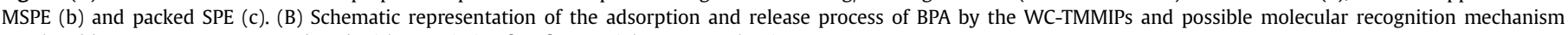
regulated by temperatures. Reproduced with permission [196]. Copyright @ 2016 Elsevier.

higher stability in the water than normal MIPs. Also, the performances of the non-specific adsorption of proteins on the MIPs and RAM-MIPs were assessed and the accumulative adsorption of proteins for MIPs and RAM-MIPs were $23.9 \%$ and $2.7 \%$, respectively, in which RAM-MIPs showed the lower adsorption of proteins.

Souza et al. constructed RAM-MIPs via in situ polymerization for SPME of parabens in breast milk samples followed by UHPLC-MS/ MS determination [210]. The RAM-MIP phase was modified with a hydrophilic external layer and applied for direct determination of parabens in breast milk samples. The SEM image showed the RAM-
MIPs had rough surface with non-uniform particle size and the thickness of the inner MIP layer was estimated near $8.5 \mathrm{~nm}$. The obtained IF values were $11,8,8$ and 14 for methylparaben, ethylparaben, propylparaben, and butylparaben, respectively. The peak areas corresponding to the endogenous compounds in the blank milk samples analyzed with the RAM-MIP capillary were lower (33\%) than the peak areas observed by using the RAM-MIP phase. The hydrophilic external layer created a chemical diffusion barrier (non-adsorptive network) which excluded the matrix components. The linear range of the proposed method was $3-400 \mathrm{ng} \mathrm{mL}^{-1}$ with 
$\mathrm{R}^{2}$ more than 0.99 . Also, the LOQs of the method were $3 \mathrm{ng} \mathrm{mL}^{-1}$ for butylparaben and $10 \mathrm{ng} \mathrm{\textrm {mL } ^ { - 1 }}$ for methyl, ethyl and propyl parabens. Different approaches have been reported for determination of parabens in milk samples [211], and in the most of them, at first, the proteins were precipitated by organic solvents like methanol or acetonitrile to minimize the interference. The main concern about these methods is consuming a large volume of organic solvent and losing analyte molecules during several pretreatment steps. However, RAM-MIPs based methods allow direct injection of biological samples into analytical systems, dismissing the need for prior treatment.

He et al. constructed RAM-MIPs for extraction of organophosphorus pesticides from the honey sample [212]. Different ratios of the template to functional monomer were evaluated and the RAMMIPs at the ratio of $1: 1$ showed the maximum adsorption capacity $\left(7.8 \mathrm{mg} \mathrm{g}^{-1}\right)$. The prepared RAM-MIPs were compared with the MIPs, C18, SPE, and Florisil SPE to determine six kinds of organophosphorus pesticides in the honey. There are a huge number of macromolecules in the honey samples and should be removed before the procedures of traditional SPE. However, this step can be completely omitted before RAM-MIP-SPE. The obtained extraction recoveries for RAM-MIPs were better than MIPs, $\mathrm{C}_{18}$, SPE, and Florisil SPE, which demonstrated the high efficiency of RAM-MIPs for determination of organophosphorus pesticides. The proposed method showed a linear range of $0.01-1.0 \mu \mathrm{g} \mathrm{mL}^{-1}$ with LODs of $0.0005-0.0019 \mu \mathrm{g} \mathrm{mL}^{-1}$ and RSDs of $2.26-4.81 \%$.

Du et al. synthesized excellent RAM-MIPs and applied them for isolation of 2-methoxyestradiol in plasma samples [213]. The RAMMIPs were synthesized by precipitation polymerization combined with the use of hydrophilic external layer and dummy imprinting strategy. During the extraction process, the analyte 2methoxyestradiol was sufficiently cleaned from the matrix with no interferences for further HPLC analysis. Water dispersion and protein binding experiments were carried out for assessment of the water compatibility and hydrophilic properties of prepared MIPs and RAM-MIPs. The RAM-MIPs exhibited better performance in water solution than MIPs. The accumulative adsorption values of proteins for the four polymers namely MIPs, NIPs, RAM-MIPs, and RAM-NIPs were $60.6 \%, 61.7 \%, 6.5 \%$, and $6.3 \%$, respectively; RAMMIPs and RAM-NIPs represented much lower adsorption of proteins. And the RAM-MIPs based method obtained excellent linearity from 0.06 to $20 \mu \mathrm{g} \mathrm{mL}^{-1}$ with the correlation coefficient of 0.9991 .
To sum up, in Fig. 12, the limitations of MIPs, proposed eight MIT strategies for addressing the defects, advantages and disadvantages of each strategy technology are briefly shown. In order to better understand the applicability of the MIPs compared with other adsorbents, typical comparison between different methods for the determination of various target analytes is done and results are presented in Table 3. For fair judgment, we have tried to choose the methods in which the sensitivity of their detectors is almost similar for same analyte. As seen, the analytical performances of the most MI-SPE approaches, namely linear range, LOQ and recovery, are better than that of other techniques. This typical comparison is a small evidence for the superiority of the MIPs than other adsorbents. Must bear in mind, other noted adsorbents suffer from lack of selectivity. However, some challenges such as consumption of hazard reagents and template molecules, difficulty in controlling polymerization conditions, and low batch to batch reproducibility, prevent the full replacement of MIPs with other adsorbents.

\section{Potential of MIPs for implementation in routine laboratory activities and scale-up}

Currently, MIPs are utilized in broad fields such as analytical separation, artificial antibody, sensing, in-vivo application and etc. due to exceptional properties of these materials such as high selectivity, cost-effectiveness, long-term storage stability, high chemical and physical stability in different media. Up to now, many efforts have been done for commercialization and industrial use of MIPs and also resulting in the production of usable products for both industrial and laboratory applications. On this matter, Claude et al. [214] investigated the application of a synthesized MIPs as a new and available commercial sorbent for selective determination of glyphosate (GLY) and its main metabolite, aminomethylphosphonic acid (AMPA) in different environmental waters. The results showed that the applied method using proposed sorbent (AFFINIMIP ${ }^{\circ}$ SPE Glyphosate-AMPA) $(250 \mathrm{mg} / 3 \mathrm{~mL}$ ) was rapid and had high ability for determination of GLY and AMPA from different water samples by UPLC-MS/MS. The method had the linearity up to $750 \mathrm{ng} \mathrm{L}^{-1}$ with $\mathrm{R}^{2}$ higher than 0.98. Pavlović et al. [215] applied a commercial SPE cartridge namely MIP ${ }_{\text {MAA-Oasis }}$ HLB $(400 \mathrm{mg} / 6 \mathrm{~mL})$ for extraction and determination of eight pharmaceuticals from water samples. Indeed, the used MIP cartridge was a combination of commercial sorbent and synthesized
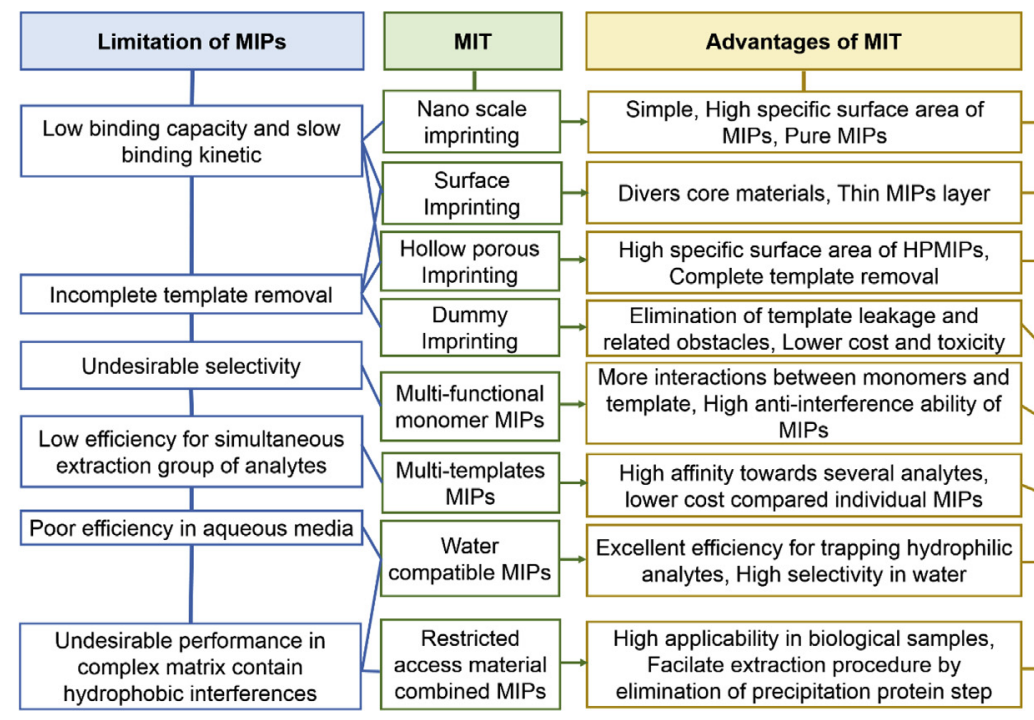

\begin{tabular}{|c|}
\hline Disadvantages of MIT \\
\hline 1 \\
\hline $\begin{array}{l}\text { Uncontrollable particle size, Agglomeration of particles, } \\
\text { High porogen volume consumption, Remaining of residual template }\end{array}$ \\
\hline $\begin{array}{l}\text { Uncontoroble MIPs layer thickness, low surface area core, non-specific } \\
\text { adsorption by core, template leakage }\end{array}$ \\
\hline $\begin{array}{l}\text { Sophisticate synthesis, High solvent consumption, damage of thin } \\
\text { HPMIPs in harsh conditions, HPMIPs suspended on the top of sample } \\
\text { solution }\end{array}$ \\
\hline Limit dummy molecules, Lower selectivity compared template imprinting \\
\hline $\begin{array}{l}\text { Selecting of monomers and how to wisely combine is arduous, disability } \\
\text { for template molecules with lack of functional group }\end{array}$ \\
\hline $\begin{array}{l}\text { Lower binding capacity for each analyte compared individual MIPs, } \\
\text { Optimization of reagents is sophisticated, Heterogeneous binding sites }\end{array}$ \\
\hline $\begin{array}{l}\text { Limit hydrophilic materials, Sophisticate several synthesis steps, Non- } \\
\text { specific adsorption, Heterogeneity of binding sites }\end{array}$ \\
\hline $\begin{array}{l}\text { Synthesis procedure is complex, Hydrophilic compounds for linking with } \\
\text { MIPs is limit }\end{array}$ \\
\hline
\end{tabular}

Fig. 12. Limitations of MIPs, proposed MIT strategies for addressing the defects, advantages and disadvantages of each strategy-technology. 
Table 3

Comparison between MI-SPE and other adsorbent-SPE techniques for different analytes.

\begin{tabular}{|c|c|c|c|c|c|c|c|c|c|c|c|}
\hline Type & Analyte & Sorbent & MIT strategy & $\begin{array}{l}\text { Adsorption } \\
\text { capacity } \\
\left(\mathrm{mg} \mathrm{g}^{-1}\right)\end{array}$ & SPE mode & $\begin{array}{l}\text { Detection } \\
\text { technique }\end{array}$ & $\begin{array}{l}\text { Linear } \\
\text { range } \\
\left(\mu \mathrm{g} \mathrm{L}^{-1}\right)\end{array}$ & $\begin{array}{l}\text { LOQ } \\
\left(\mu \mathrm{g} \mathrm{L}^{-1}\right)\end{array}$ & $\begin{array}{l}\text { Real } \\
\text { sample }\end{array}$ & $\begin{array}{l}\text { Recovery } \\
(\%)\end{array}$ & Ref. \\
\hline \multirow[t]{10}{*}{ Drug } & \multirow[t]{2}{*}{ Cocaine } & MIPs & Monolithic & 0.08 & $\mu-\mathrm{SPE}$ & HPLC-MS/MS & - & 0.2 & Plasma & $95.8-105.0$ & [241] \\
\hline & & $\mathrm{CCT}$ & - & - & MEFAE & UHPLC-MS/MS & $1-100$ & 0.8 & Saliva & $56.0-70.0$ & [242] \\
\hline & \multirow[t]{2}{*}{ Sulfonamide } & MISG & Monolithic & - & $\mathrm{d}-\mathrm{SPE}$ & HPLC-UV & $1-500$ & 0.2 & Water & $80.0-96.0$ & [243] \\
\hline & & COF@MOF & - & 7.2 & PT-SPE & HPLC-VWD & $10-2000$ & - & Water & $68.9-103.8$ & [244] \\
\hline & \multirow[t]{2}{*}{ Valsartan } & HP-MINs & HPMIP & 46.32 & d-SPE & HPLC-UV & $5-2000$ & 4.9 & Urine & $87.5-97.0$ & [141] \\
\hline & & - & - & - & SPE & HPLC-DAD & $85-15000$ & 85.0 & Urine & $92.8-98.8$ & [245] \\
\hline & \multirow[t]{2}{*}{ Ketamine } & MIPs & Monolithic & - & SPE & LC-MS/MS & $0.1-10$ & 0.18 & Hair & 86.1 & [246] \\
\hline & & $\mathrm{C}_{18} / \mathrm{BSAP}$ & - & - & SPME & ESI-MS & $0.1-500$ & 0.1 & Urine & $90.8-109.4$ & [247] \\
\hline & \multirow[t]{2}{*}{ Metformin } & MIPs & $\begin{array}{l}\text { Surface } \\
\text { imprinting }\end{array}$ & 80.0 & d-SPE & HPLC-UV & $0.5-50$ & 0.44 & Urine & $\geq 97$ & [248] \\
\hline & & $\begin{array}{l}\text { Strata }{ }^{\mathrm{TM}} X \\
\text { cartridge } / C_{18}\end{array}$ & - & - & SPE & HPLC-MS/MS & $25-2000$ & 25.0 & Plasma & $85.0-106.16$ & [249] \\
\hline \multirow[t]{10}{*}{$\begin{array}{l}\text { Food } \\
\text { contaminant }\end{array}$} & \multirow[t]{2}{*}{ Acrylamide } & MIPs & $\begin{array}{l}\text { Dummy/water } \\
\text { compatible }\end{array}$ & 24.1 & d-SPE & HPLC-UV & $5-5000$ & 4.4 & Biscuit & $86.0-98.3$ & [125] \\
\hline & & EMRL & - & - & d-SPE & HPLC-MS/MS & - & 8.3 & $\mathrm{HPF}$ & $87.3-103.3$ & [250] \\
\hline & \multirow[t]{2}{*}{ Aflatoxins } & MIPs & Monolithic & & $\mu$-SPE & LC-MS/MS & $2.5-100$ & $1.3-3.5$ & Fish feed & $80.0-100.0$ & [73] \\
\hline & & $\begin{array}{l}\text { polyurethane-GO } \\
\text { nanofiber }\end{array}$ & - & - & SPE/DLLME & HPLC-FD & $0.3-1000$ & $0.3-0.5$ & Soybean & $76.0-101.0$ & [251] \\
\hline & \multirow[t]{2}{*}{ Nitrophenols } & MIPs & $\begin{array}{l}\text { Surface } \\
\text { imprinting }\end{array}$ & - & SPME & HPLC-UV & $10-1000$ & - & Water & - & [252] \\
\hline & & $\begin{array}{l}\text { A monolithic graphitic } \\
\text { carbon nitride/ } \\
\text { polyethersulfone } \\
\text { composite }\end{array}$ & - & - & SPME & HPLC-UV & $2-2500$ & $2-5$ & $\begin{array}{l}\text { Refinery } \\
\text { waste } \\
\text { water }\end{array}$ & $72.0-108.0$ & [253] \\
\hline & \multirow[t]{2}{*}{ Carbamates } & MIPs & $\begin{array}{l}\text { Surface } \\
\text { imprinting }\end{array}$ & - & d-SPE & HPLC-UV & $40-10000$ & - & Fruit & $90.5-98.6$ & [254] \\
\hline & & $\begin{array}{l}\text { CTAB modified Zeolite } \\
\text { NaY }\end{array}$ & - & - & $\mu-S P E$ & HPLC-DAD & $4-4000$ & 4 & Water & $79.5-124.0$ & [255] \\
\hline & \multirow{2}{*}{$\begin{array}{l}\text { Triazine } \\
\text { herbicides }\end{array}$} & MIPs & Multi-template & - & SPE & LC-MS/MS & $10-200$ & - & Agro-products & $61.3-105.9$ & [256] \\
\hline & & $\begin{array}{l}\text { MIL-101(Cr)/ } \\
\text { MWCNTs- } \\
\text { functionalized } \\
\text { melamine sponges }\end{array}$ & - & - & d-SPE & HPLC-MS/MS & $0.5-50$ & $0.01-0.04$ & Corn samples & $76.52-118.86$ & [257] \\
\hline \multirow[t]{10}{*}{$\begin{array}{l}\text { Environmental } \\
\text { pollution }\end{array}$} & \multirow[t]{2}{*}{ PAHs } & MIPs & $\begin{array}{l}\text { Surface } \\
\text { imprinting }\end{array}$ & - & d-SPE & APGC-MS/MS & $2-50000$ & $2-200$ & Water & $70.0-130.0$ & [258] \\
\hline & & Carbon foam & - & - & $\mu$-SPE & GC-MS/MS & $25-100$ & - & Waste water & $91.8-102.0$ & [259] \\
\hline & \multirow[t]{2}{*}{ Bisphenol A } & MIPs & Dummy & - & $\mu-S P E$ & HPLC-UV & $0.01-15$ & 0.01 & Water & $65.56-88.84$ & [238] \\
\hline & & MOFs & - & 252.5 & d-SPE & HPLC-UV & $0.25-10$ & $0.11-0.24$ & $\begin{array}{l}\text { Environmental } \\
\text { water }\end{array}$ & - & [260] \\
\hline & \multirow[t]{2}{*}{ Sudan dyes } & MIPs & $\begin{array}{l}\text { Surface } \\
\text { imprinting }\end{array}$ & - & d-SPE & HPLC-UV & $10-10000$ & $6.0-21.2$ & Chili powder & $74.1-93.3$ & [261] \\
\hline & & MOF-ZnO composite & - & - & SPME & HPLC-UV & $0.02-20$ & - & Water & $83.5-95.0$ & [262] \\
\hline & \multirow[t]{2}{*}{ Carboxylic acid } & MIPs & Dummy & 1.2 & SPE & LC-MS/MS & $1-50$ & - & Milk & $75.3-89.8$ & [263] \\
\hline & & $\begin{array}{l}\text { Surfactant- templated } \\
\text { ordered mesoporous } \\
\text { material }\end{array}$ & - & - & $\mu-S P E$ & LC-MS/MS & - & - & Water & $64.0-127.0$ & [264] \\
\hline & \multirow[t]{2}{*}{ Hippuric acid } & MIPs & RAM-MIP & 68.5 & SPE & HPLC-UV & $0.3-7500$ & 0.25 & Urine & $88.0-104.0$ & [207] \\
\hline & & $\begin{array}{l}\mathrm{Fe}_{3} \mathrm{O}_{4} @ \mathrm{SiO}_{2} @ \text { sodium } \\
\text { dodecyl sulfate }\end{array}$ & - & - & d-SPE & HPLC-UV & $5-4000$ & 5 & Urine & $96.0-103.3$ & [265] \\
\hline \multirow[t]{6}{*}{ Macromolecule } & \multirow[t]{2}{*}{$\begin{array}{l}\text { Human serum } \\
\text { albumin }\end{array}$} & MIPs & $\begin{array}{l}\text { Surface } \\
\text { imprinting }\end{array}$ & 66.23 & d-SPE & HPLC-UV & - & - & Urine & $91.95-97.8$ & [266] \\
\hline & & $\mathrm{Fe}_{3} \mathrm{O}_{4} @ G O-D E S$ & - & 110.0 & d-SPE & $\begin{array}{l}\text { UV-vis } \\
\text { spectrophotometer }\end{array}$ & - & - & Urine & $\geq 90.9$ & [267] \\
\hline & \multirow[t]{2}{*}{ Glycopeptide } & MIPs & $\begin{array}{l}\text { Composite } \\
\text { MIP }\end{array}$ & 15.0 & d-SPE & LC-MS/MS & - & - & Human serum & - & [132] \\
\hline & & $\begin{array}{l}\text { Dendritic mesoporous } \\
\text { silica nanoparticles }\end{array}$ & - & 300.0 & SPE & LC-MS/MS & - & - & Human serum & $\geq 90.0$ & [268] \\
\hline & \multirow[t]{2}{*}{ Lysozyme } & MIPs & $\begin{array}{l}\text { Surface } \\
\text { imprinting }\end{array}$ & 74.66 & - & - & - & - & $\begin{array}{l}\text { Chicken } \\
\text { egg white }\end{array}$ & 166.36 & [269] \\
\hline & & $\begin{array}{l}\text { Carboxyl- } \\
\text { functionalized porous } \\
\beta \text {-cyclodextrin } \\
\text { polymer }\end{array}$ & - & 1520 & d-SPE & HPLC-UV & - & - & Egg white & - & [270] \\
\hline
\end{tabular}

APGC-MS/MS: gas chromatography with an atmospheric pressure chemical ionization tandem mass spectrometry.

MIPs. The MIPs were synthesized via multi-functional monomer imprinting strategy and then combined with commercial cartridge (Oasis HLB) to improve the performances of the commercial cartridge.

Poma et al. [216] presented a reusable solid-phase template approach that was fully compatible with automation for the synthesis of MIP nanoparticles (MIP-NPs) and their precise manufacture using a prototype automated UV photochemical reactor, as illustrated in Fig. 13. The well-synthesized MIP-NPs were applied for more than 30 repeated cycles without obvious loss of performance. As seen from the figure, the imprinting process was automated by using syringe pumps to regulate the flow of polymerization mixtures and elution solvents. UV irradiation was also used for polymerization at low temperatures which allowed the formation of stable complexes between the immobilized template and the functional monomer while iniferter-type initiators 


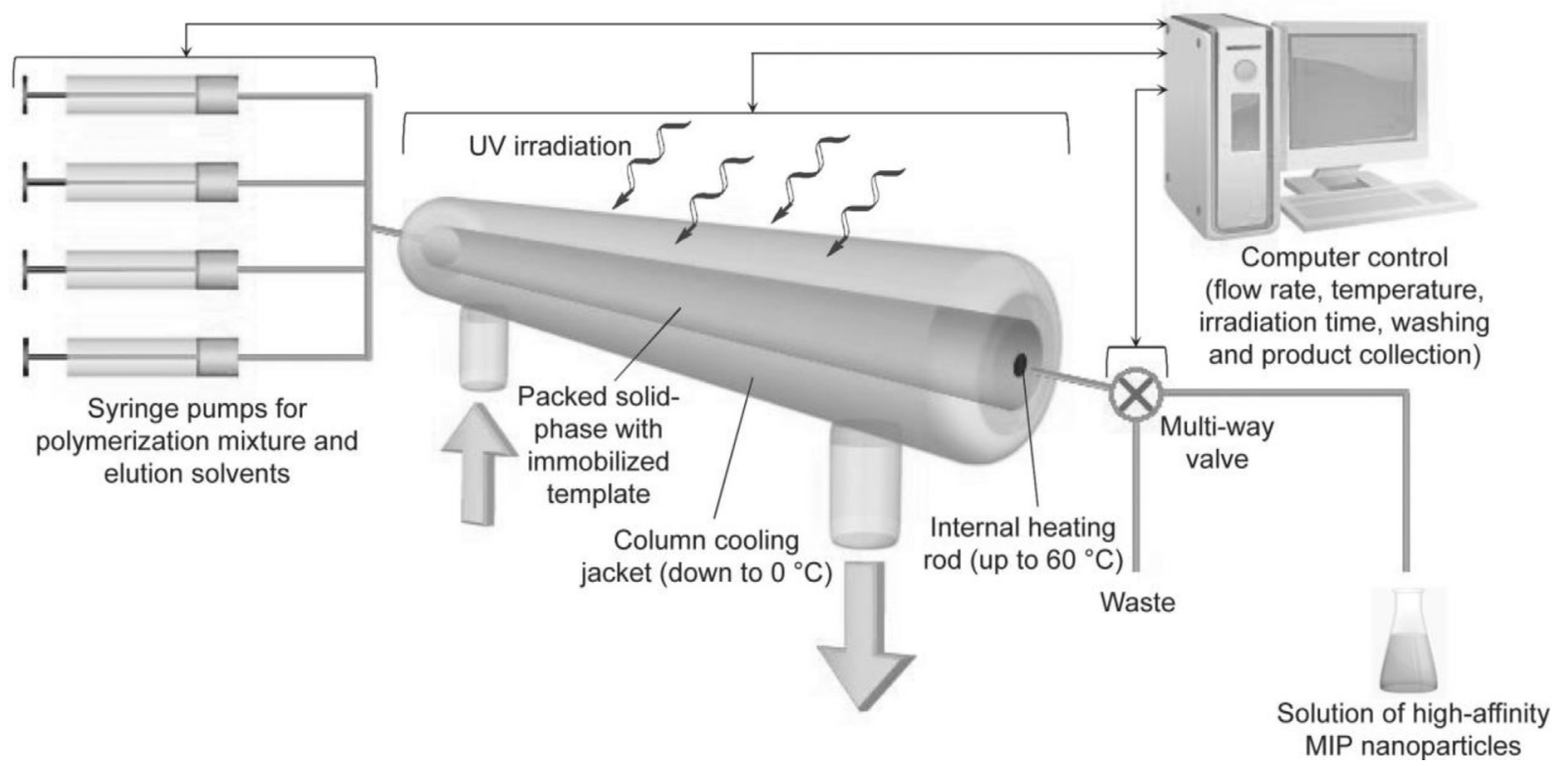

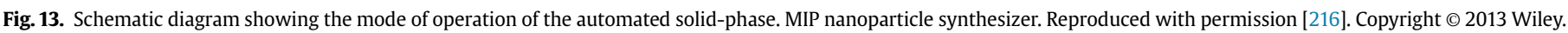

controlled the polymerizations. When polymerization accrued at the low temperature $\left(4-15^{\circ} \mathrm{C}\right)$ with fresh solvent, the imprinted particles were adhered on the solid phase. Therefore, low-affinity particles, smaller polymer chains, un-polymerized residues, and impurities could be easily removed. To elute high affinity MIP-NPs, the temperature was enhanced to $60^{\circ} \mathrm{C}$, since the strength of interactions was decreased and the exchange rate of the MIP-NPs with the solid phase was increased. When the increasing of the temperature was not sufficient to elute MIP-NPs, auxiliary reagents such as formic acid was used. The automated reactor was upgraded with a shaking mechanism to ensure adequate mixing of the polymerization mixture and an $\mathrm{N}_{2}$ inlet to flush the reactor before polymerization and to force out the liquid. By the proposed method, all binding sites were located on the surface of the particles and had the same orientation, which can improve the homogeneity of binding sites and their accessibility compare to traditional MIP-NPs synthesis methods. This study was the first report for the automated synthesis of MIP-NPs with size, specificity and solubility characteristics comparable to antibodies, suitable for industrial manufacturing. Automation allows the reactor to operate $24 \mathrm{~h}$, eliminating human error and operator fatigue and ensures precisely controlled batch-to-batch reproducibility. Moreover, the synthesis and subsequent affinity purification can easily be performed using computer controlled instruments (Fig. 13), which are applicable in industrial manufacturing [98].

Berghaus and co-workers established a scalable process to produce surface imprinted MIPs with high yield by using a solid template where the analyte was conjugated to magnetic NPs [217]. Therefore, molecular imprinting could be done in a template free form, overcoming leaching of the template molecule during further applications. These nano-sized magnetic placeholder templates were used in a RAFT mediated core-shell polymerization in order to generate functionalized nano-composites. In the first step, the monomers were polymerized in the presence of the RAFT agent coupled silica NPs and the solid template NPs. Afterward, the magnetic template and the adhered MIP core-shell NPs were removed, followed by repetitive washing-centrifugation cycles, removing unreacted monomer and oligomer. Finally, the template could be removed with acidified methanol, releasing the high affinity MIP core-shell NPs. Regarding the potential application in large scale production, the possible reuse of the magnetic placeholder template is crucial. The overall gravimetric yield of MIP-NPs was found to be around $9 \%$ assuming quantitative transformation of monomer to core-shell polymer layer. Obviously, the imprinted core-shell NPs were adhered more strongly to the solid template, compared to non-imprinted or hardly imprinted NPs. This technique was supposed to enable method scalability and parallel synthesis. In parallel to these works, other scientists suggested different approaches for the preparation of MIPs in large scale [218,219].

To date, several MIP based products for a wide variety of templates are commercially available. There are two different applications where MIP products are on the market: separation and sensing. The former are commercialized by the Swedish company Biotage and by the French PolyIntell [98]. Their product assortment includes matrix material as well as assembled SPE columns which are mainly suited for biomedical and food analysis. These enable the separation of mycotoxins (toxins released by fungi; a specific one or different combinations of), BPA (a common monomer in the polymer industry which is suspected to interfere with the hormone system), different hormones, and other clinically relevant drugs. US Company Raptor is the first company that commercialized MIP based sensors in the world. Their used templates include explosives, toxins, biochemical agents, and pesticides. Their sold products include sensing devices, in which fluoresce is in the presence of the template and also MIP based soluble powders. These have been adhered to hand wipes or provided in the form of a paint or a spray. The solution can also be applied onto products like tickets or security badges. The presence of the template is indicated by a color change which is visible by the naked eye. Although there are many potential applications for imprinting macromolecules so far, to the best of our knowledge, all templates in commercially available MIPs are small molecules.

\section{Conclusions and perspectives}

This review presents a comprehensive summary of the current status, challenges, and new strategies for improving MIPs' performances and highlighting the applications in diverse SPE modes in the recent five years. As evidenced, an extensive tendency has been 
seen in MIPs applications for sample pretreatment, because of their supreme potentials as versatile receptors for specific recognition of target analytes. Despite noteworthy achievements by MIT, still some essential development challenges need to be addressed and accordingly promising perspectives can be proposed, as follows.

(1) To design novel qualified functional monomers to interact with a template molecule without functional groups is highly desirable. (2) It is required to amend physical and thermal stability of MIPs to enhance recyclability, especially in SPME while the adsorbed analytes are thermally desorbed in high temperature. (3) Removing template molecules is time consuming and needs a high volume of solvent. An alternate approach is a demand for fast and complete template removal without consumption of solvent, such as using high temperature instead of solvent for template removal. (4) Green and environmentally friendly chemicals could be used instead of toxic and hazard reagents for the preparation of MIPs. (5) Although a few papers reported the application of MIPs for chiral compounds separation, there is very little progress in this regard and more attention is needed. (6) It is expected to exploit new synthesis approaches for higher binding capacity, faster binding kinetics and most importantly super selectivity. (7) Performing the imprinting process in aqueous media is to increase the hydrophilicity of the MIPs and to minimize hydrophobic interferences. (8) It is highly demanded to construct and commercialize excellent SPE and chromatography columns packed with MIPs which are comparable with or better than current commercial ones.

\section{Acknowledgments}

This work was financially supported by the Chinese Academy of Sciences President's International Fellowship Initiative (2019PC0050, 2020PC0083), the National Natural Science Foundation of China (21876199, 21804010, 41776110, 21976209) and the Taishan Scholar Project Special Funding (ts20190962).

\section{References}

[1] D. Wianowska, M. Gil, New insights into the application of MSPD in various fields of analytical chemistry, TrAC, Trends Anal. Chem. 112 (2019) 29-51.

[2] B. Lin, X. Lu, N. Li, N. Xu, J.M. Lin, Effect of Dai-Bai-Jie on the proliferation and migration of the A549 cells, Chin. Chem. Lett. 31 (2020) 476-478.

[3] S. Tang, H. Zhang, H.K. Lee, Advances in sample extraction, Anal. Chem. 88 (2016) 228-249.

[4] S.X.L. Goh, B.H.D. Chong, H.K. Lee, Fully automated water sampling-surfactant-enhanced membrane bag liquid-phase microextraction-ultrahigh performance liquid chromatography-mass spectrometry, Anal. Chem. 7 (2020) 5362-5369.

[5] E. Javier Carrasco-Correa, P. Kubán, D.J. Cocovi-Solberg, M. Miró, Fully automated electric-field-driven liquid phase microextraction system with renewable organic membrane as a front end to high performance liquid chromatography, Anal. Chem. 91 (2019) 10808-10815.

[6] G. Berger, V. Nolleau, C. Picou, M. Perez, A. Ortiz-Julien, M. Brulfert, C. Camaraca, A. Bloem, Dispersive liquid-liquid microextraction for the quantitation of terpenes in wine, J. Agric. Food Chem. (2020). https://doi.org/ 10.1021/acs.jafc.9b08222.

[7] B. Kim, S. Oh, D. You, S. Choi, Microfluidic pipette tip for high-purity and high-throughput blood plasma separation from whole blood, Anal. Chem. 89 (2017) 1439-1444.

[8] Q. Su, P. Vera, C. Nerin, Direct immersion-solid-phase micro-extraction coupled to gas chromatography-mass spectrometry and response surface methodology for non-target screening of (semi-) volatile migrants from food contact materials, Anal. Chem. 7 (2020) 5577-5584.

[9] H. Birch, N.I. Kramer, P. Mayer, Time-resolved freely dissolved concentrations of semivolatile and hydrophobic test chemicals in in vitro assays-measuring high losses and crossover by headspace solid-phase microextraction, Chem. Res. Toxicol. 32 (2019) 1780-1790.

[10] M. Yousefi, M. Rahimi-Nasrabadi, S.M. Pourmortazavi, M. Wysokowski, T. Jesionowski, H. Ehrlich, S. Mirsadeghi, Supercritical fluid extraction of essential oils, TrAC, Trends Anal. Chem. 118 (2019) 182-193.

[11] T. Devièse, A. Van Ham-Meert, V.J. Hare, J. Lundy, P. Hommel, V. Ivanovich Bazaliiskii, J. Orton, Supercritical fluids for higher extraction yields of lipids from archeological ceramics, Anal. Chem. 90 (2018) 2420-2424.
[12] N. Hamada, Y. Hashi, S. Yamaki, Y. Guo, L. Zhang, H. Li, J.M. Lin, Construction of on-line supercritical fluid extraction with reverse phase liquid chromatography-tandem mass spectrometry for the determination of capsaicin, Chin. Chem. Lett. 30 (2019) 99-102.

[13] Á. Santana-Mayor, B. Socas-Rodríguez, A.V. Herrera-Herrera, M.Á. RodríguezDelgado, Current trends in QuEChERS method. A versatile procedure for food, environmental and biological analysis TrAC-Special Issue "Green Extraction Techniques", TrAC, Trends Anal. Chem. 116 (2019) 214-235.

[14] V.C. Fernandes, W. Luts, C. Delerue-Matos, V.F. Domingues, Improved QuEChERS for analysis of polybrominated diphenyl ethers and novel brominated flame retardants in capsicum cultivars using gas chromatography, J. Agric. Food Chem. 68 (2020) 3260-3266.

[15] E.A. Nantia, D. Moreno-González, F.P. Manfo, L. Gámiz-Gracia, A.M. GarcíaCampaña, QuEChERS-based method for the determination of carbamate residues in aromatic herbs by UHPLC-MS/MS, Food Chem. 216 (2017) $334-341$.

[16] M. Li, S. Mao, S. Wang, H.F. Li, J.M. Lin, Chip-based SALDI-MS for rapid determination of intracellular ratios of glutathione to glutathione disulfide, Sci. China Chem. 62 (2019) 142-150.

[17] G.G. Nestorova, K. Hasenstein, N. Nguyen, M.A. DeCoster, N.D. Crews, Lab-ona-chip mRNA purification and reverse transcription via a solid-phase gene extraction technique, Lab Chip 17 (2017) 1128-1136.

[18] Y. Zheng, Z. Wu, J.M. Lin, L. Lin, Imitation of drug metabolism in cell coculture microcapsule model using a microfluidic chip platform coupled to mass spectrometry, Chin. Chem. Lett. 31 (2020) 451-454.

[19] X. Zhang, F. Cao, L. Wu, X. Jiang, Understanding the synergic mechanism of weak interactions between graphene oxide and lipid membrane leading to the extraction of lipids, Langmuir 35 (2019) 14098-14107.

[20] Z. Wu, N. Xu, W. Li, J.M. Lin, A membrane separation technique for optimizing sample preparation of MALDI-TOF MS detection, Chin. Chem. Lett. 30 (2019) 95-98.

[21] R. Lebl, T. Murray, A. Adamo, D. Cantillo, C.O. Kappe, Continuous flow synthesis of methyl oximino acetoacetate: accessing greener purification methods with inline liquid-liquid extraction and membrane separation technology, ACS Sustain. Chem. Eng. 7 (2019) 20088-20096.

[22] K.M. Dimpe, P.N. Nomngongo, Current sample preparation methodologies for analysis of emerging pollutants in different environmental matrices, TrAC, Trends Anal. Chem. 82 (2016) 199-207.

[23] D.E. Raynie, Modern extraction techniques, Anal. Chem. 82 (2010) 4911-4916.

[24] B.H. Fumes, M.R. Silva, F.N. Andrade, C.E.D. Nazario, F.M. Lanças, Recent advances and future trends in new materials for sample preparation, TrAC, Trends Anal. Chem. 71 (2015) 9-25.

[25] M. Polyakov, Adsorption properties and structure of silica gel, Zh. Fiz. Khim. 2 (1931) 799-805

[26] V. Pichon, F. Chapuis-Hugon, Role of molecularly imprinted polymers for selective determination of environmental pollutants-a review, Anal. Chim. Acta 622 (2008) 48-61.

[27] A. Speltini, A. Scalabrini, F. Maraschi, M. Sturini, A. Profumo, Newest applications of molecularly imprinted polymers for extraction of contaminants from environmental and food matrices: a review, Anal. Chim. Acta 974 (2017) $1-26$.

[28] L.X. Chen, X.Y. Wang, W.H. Lu, X.Q. Wu, J.H. Li, Molecular imprinting: perspectives and applications, Chem. Soc. Rev. 45 (2016) 2137-2211.

[29] L.X. Chen, S.F. Xu, J.H. Li, Recent advances in molecular imprinting technology: current status, challenges and highlighted applications, Chem. Soc. Rev. 40 (2011) 2922-2942.

[30] F. Canfarotta, L. Lezina, A. Guerreiro, J. Czulak, A. Petukhov, A. Daks, K. Smolinska-Kempisty, A. Poma, S. Piletsky, N.A. Barlev, Specific drug delivery to cancer cells with double-imprinted nanoparticles against epidermal growth factor receptor, Nano Lett. 18 (2018) 4641-4646.

[31] P. Luliński, Molecularly imprinted polymers based drug delivery devices: a way to application in modern pharmacotherapy. A review, Mater. Sci. Eng. C 76 (2017) 1344-1353.

[32] Q. Yang, J.H. Li, X.Y. Wang, H. Xiong, L.X. Chen, Ternary emission of a bluegreen-, and red-based molecular imprinting fluorescence sensor for the multiplexed and visual detection of bovine hemoglobin, Anal. Chem. 91 (2019) 6561-6568.

[33] Q. Yang, J.H. Li, X.Y. Wang, H.L. Peng, H. Xiong, L.X. Chen, Strategies of molecular imprinting-based fluorescence sensors for chemical and biological analysis, Biosens. Bioelectron. 112 (2018) 54-71.

[34] S. Muratsugu, H. Baba, T. Tanimoto, K. Sawaguchi, S. Ikemoto, M. Tasaki, Y. Terao, M. Tada, Chemoselective epoxidation of cholesterol derivatives on a surface-designed molecularly imprinted Ru-porphyrin catalyst, Chem. Commun. 54 (2018) 5114-5117.

[35] S. Muratsugu, N. Maity, H. Baba, M. Tasaki, M. Tada, Preparation and catalytic performance of a molecularly imprinted Pd complex catalyst for Suzuki cross-coupling reactions, Dalton Trans. 46 (2017) 3125-3134.

[36] C. Rossetti, M.A. Świtnicka-Plak, T.G. Halvorsen, P.A. Cormack, B. Sellergren, L. Reubsaet, Automated protein biomarker analysis: on-line extraction of clinical samples by molecularly imprinted polymers, Sci. Rep. 7 (2017) 44298.

[37] A. Martín-Esteban, Molecularly-imprinted polymers as a versatile, highly selective tool in sample preparation, TrAC, Trends Anal. Chem. 45 (2013) 169-181. 
[38] T. Zhou, L. Ding, G. Che, W. Jiang, L. Sang, Recent advances and trends of molecularly imprinted polymers for specific recognition in aqueous matrix: preparation and application in sample pretreatment, TrAC, Trends Anal. Chem. 114 (2019) 11-28.

[39] F.J. Ning, H.L. Peng, J.H. Li, L.X. Chen, H. Xiong, Molecularly imprinted polymer on magnetic graphene oxide for fast and selective extraction of $17 \beta$ estradiol, J. Agric. Food Chem. 62 (2014) 7436-7443.

[40] R.G. da Costa Silva, F. Augusto, Sol-gel molecular imprinted ormosil for solid-phase extraction of methylxanthines, J. Chromatogr. A 1114 (2006) 216-223.

[41] G. Zhu, W. Li, L. Wang, P. Wang, D. Shi, J. Wang, J. Fan, Using ionic liquid monomer to improve the selective recognition performance of surface imprinted polymer for sulfamonomethoxine in strong polar medium, J. Chromatogr. A 1592 (2019) 38-46.

[42] Y. Liu, Y. Wang, Q. Dai, Y. Zhou, Magnetic deep eutectic solvents molecularly imprinted polymers for the selective recognition and separation of protein, Anal. Chim. Acta 936 (2016) 168-178.

[43] M. Zhong, Y. Teng, S. Pang, L. Yan, X. Kan, Pyrrole-phenylboronic acid: a novel monomer for dopamine recognition and detection based on imprinted electrochemical sensor, Biosens. Bioelectron, 64 (2015) 212-218.

[44] W. Ji, L. Chen, X. Ma, X. Wang, Q. Gao, Y. Geng, L. Huang, Molecularly imprinted polymers with novel functional monomer for selective solidphase extraction of gastrodin from the aqueous extract of Gastrodia elata, J. Chromatogr. A 1342 (2014) 1-7.

[45] W. Ji, M. Zhang, D. Wang, X. Wang, J. Liu, L. Huang, Superhydrophilic molecularly imprinted polymers based on a water-soluble functional monomer for the recognition of gastrodin in water media, J. Chromatogr. A 1425 (2015) 88-96.

[46] J. Liu, K. Yang, Q. Deng, Q. Li, L. Zhang, Z. Liang, Y. Zhang, Preparation of a new type of affinity materials combining metal coordination with molecular imprinting, Chem. Commun. 47 (2011) 3969-3971.

[47] J. Zhou, Y. Wang, J. Bu, B. Zhang, Q. Zhang, $\mathrm{Ni}^{2+}$-BSA directional coordinationassisted magnetic molecularly imprinted microspheres with enhanced specific rebinding to target proteins, ACS Appl. Mater. Interfaces 11 (2019) $25682-25690$

[48] K.Z. Chin, S.M. Chang, $\mathrm{SiO}_{2}$-Coated molecularly imprinted copolymer nanostructures for the adsorption of bisphenol A, ACS Appl. Nano Mater. Interfaces 2 (2019) 89-99.

[49] Y.K. Lv, L.M. Wang, L. Yang, C.X. Zhao, H.W. Sun, Synthesis and application of molecularly imprinted poly (methacrylic acid)-silica hybrid composite material for selective solid-phase extraction and high-performance liquid chromatography determination of oxytetracycline residues in milk J. Chromatogr. A 1227 (2012) 48-53.

[50] D. Clausen, J. Visentainer, C. Tarley, Development of molecularly imprinted poly (methacrylic acid)/silica for clean-up and selective extraction of cholesterol in milk prior to analysis by HPLC-UV, Analyst 139 (2014) 5021-5027.

[51] D.N. Clausen, I.M.R. Pires, C.R.T. Tarley, Improved selective cholesterol adsorption by molecularly imprinted poly (methacrylic acid)/silic $\left(\mathrm{PMAA}-\mathrm{SiO}_{2}\right.$ ) hybrid material synthesized with different molar ratios, Mater Sci. Eng. C 44 (2014) 99-108.

[52] Q. Liu, Y. Zhao, J. Pan, B. Van der Bruggen, J. Shen, A novel chitosan base molecularly imprinted membrane for selective separation of chlorogenic acid, Separ. Purif. Technol. 164 (2016) 70-80.

[53] Z. Zhou, K. Cui, Y. Mao, W. Chai, N. Wang, Z. Ren, Green preparation of dtryptophan imprinted self-supported membrane for ultrahigh enantioseparation of racemic tryptophan, RSC Adv. 6 (2016) 109992-110000.

[54] H. Zheng, M. Yoshikawa, Molecularly imprinted cellulose membranes for pervaporation separation of xylene isomers, J. Membr. Sci. 478 (2015) 148-154.

[55] L.Y. Wang, J.H. Li, J.N. Wang, X.T. Guo, X.Y. Wang, J. Choo, L.X. Chen, Green multi-functional monomer based ion imprinted polymers for selective removal of copper ions from aqueous solution, J. Colloid Interface Sci. 541 (2019) 376-386

[56] Y. Geng, M. Guo, J. Tan, S. Huang, Y. Tang, L. Tan, Y. Liang, A fluorescent molecularly imprinted polymer using aptamer as a functional monomer for sensing of kanamycin, Sensor. Actuator. B Chem. 268 (2018) 47-54.

[57] D. Xiao, Y. Jiang, Y. Bi, Molecularly imprinted polymers for the detection of illegal drugs and additives: a review, Microchim. Acta 185 (2018) 247.

[58] M.F. Jia, Z. Zhang, J.H. Li, X. Ma, L.X. Chen, X.B. Yang, Molecular imprinting technology for microorganism analysis, TrAC, Trends Anal. Chem. 106 (2018) 190-201.

[59] S. Ansari, M. Karimi, Novel developments and trends of analytical methods for drug analysis in biological and environmental samples by molecularly imprinted polymers, TrAC, Trends Anal. Chem. 89 (2017) 146-162.

[60] M. Dinc, C. Esen, B. Mizaikoff, Recent advances on core-shell magnetic molecularly imprinted polymers for biomacromolecules, TrAC, Trends Anal. Chem. 114 (2019) 202-217.

[61] S. Ansari, Application of magnetic molecularly imprinted polymer as a versatile and highly selective tool in food and environmental analysis: recent developments and trends, TrAC, Trends Anal. Chem. 90 (2017) 89-106.

[62] S. Huang, J. Xu, J. Zheng, F. Zhu, L. Xie, G. Ouyang, Synthesis and application of magnetic molecularly imprinted polymers in sample preparation, Anal. Bioanal. Chem. 410 (2018) 3991-4014.

[63] M.M. Moein, A. Abdel-Rehim, M. Abdel-Rehim, Recent applications of molecularly imprinted sol-gel methodology in sample preparation, Molecules 24 (2019) 2889
[64] J.E. Lofgreen, G.A. Ozin, Controlling morphology and porosity to improve performance of molecularly imprinted sol-gel silica, Chem. Soc. Rev. 43 (2014) 911-933.

[65] R. Keçili, C.M. Hussain, Recent progress of imprinted nanomaterials in analytical chemistry, Int. J. Anal. Chem. (2018) 8503853. https://doi.org/ $10.1155 / 2018 / 8503853$

[66] Z.H. Wei, L.N. Mu, Y.P. Huang, Z.S. Liu, Imprinted monoliths: recent significant progress in analysis field, TrAC, Trends Anal. Chem. 86 (2017) 84-92.

[67] J. Tan, Z.T. Jiang, R. Li, X.P. Yan, Molecularly-imprinted monoliths for sample treatment and separation, TrAC, Trends Anal. Chem. 39 (2012) 207-217.

[68] X. Wang, P. Huang, X. Ma, X. Du, X. Lu, Enhanced in-out-tube solid-phase microextraction by molecularly imprinted polymers-coated capillary followed by HPLC for Endocrine Disrupting Chemicals analysis, Talanta 194 (2019) 7-13.

[69] S. Huang, G. Chen, N. Ye, X. Kou, F. Zhu, J. Shen, G. Ouyang, Solid-phase microextraction: an appealing alternative for the determination of endogenous substances-A review, Anal. Chim. Acta 1077 (2019) 67-86.

[70] H. Asiabi, Y. Yamini, S. Seidi, F. Ghahramanifard, Preparation and evaluation of a novel molecularly imprinted polymer coating for selective extraction of indomethacin from biological samples by electrochemically controlled intube solid phase microextraction, Anal. Chim. Acta 913 (2016) 76-85.

[71] K. Poniedziałek, K. Bielicka-Daszkiewicz, Application of micro-solid-phase extraction for determination of released dental fillings components in artificial saliva solution, Anal. Chim. Acta 1041 (2018) 146-155.

[72] S. Aihebaier, T. Muhammad, A. Wei, A. Mamat, M. Abuduaini, P. Pataer, A. Yigaimu, A. Yimit, Membrane-protected molecularly imprinted polymer for the microextraction of indole-3-butyric acid in mung bean sprouts, ACS Omega 4 (2019) 16789-16793.

[73] G.D.T.M. Jayasinghe, R. Domínguez-González, P. Bermejo-Barrera, A. MoredaPiñeiro, Ultrasound assisted combined molecularly imprinted polymer for the selective micro-solid phase extraction and determination of aflatoxins in fish feed using liquid chromatography-tandem mass spectrometry, J. Chromatogr. A 1609 (2020) 460431.

[74] M. Sajid, C. Basheer, A. Alsharaa, K. Narasimhan, A. Buhmeida, M. Al Oahtani, M.S. Al-Ahwal, Development of natural sorbent based micro-solid-phase extraction for determination of phthalate esters in milk samples, Anal. Chim. Acta 924 (2016) 35-44.

[75] A. González, J. Avivar, F. Maya, C. Palomino Cabello, G. Turnes Palomino, V. Cerdà, In-syringe dispersive $\mu$-SPE of estrogens using magnetic carbon microparticles obtained from zeolitic imidazolate frameworks, Anal. Bioanal. Chem. 409 (2017) 225-234.

[76] J. Sánchez-González, M.J. Tabernero, A.M. Bermejo, P. Bermejo-Barrera, A. Moreda-Piñeiro, Porous membrane-protected molecularly imprinted polymer micro-solid-phase extraction for analysis of urinary cocaine and its metabolites using liquid chromatography - tandem mass spectrometry, Anal. Chim. Acta 898 (2015) 50-59.

[77] Y. Tang, J. Lan, X. Gao, X. Liu, D. Zhang, L. Wei, Z. Gao, J. Li, Determination of clenbuterol in pork and potable water samples by molecularly imprinted polymer through the use of covalent imprinting method, Food Chem. 190 (2016) 952-959.

[78] Z. Xu, L. Ding, Y. Long, L. Xu, L. Wang, C. Xu, Preparation and evaluation of superparamagnetic surface molecularly imprinted polymer nanoparticles for selective extraction of bisphenol A in packed food, Anal. Methods 3 (2011) 1737-1744.

[79] M.R. Gama, F.R.P. Rocha, C.B.G. Bottoli, Monoliths: synthetic routes, functionalization and innovative analytical applications, TrAC, Trends Anal. Chem. 115 (2019) 39-51.

[80] A. El-Beqqali, M. Abdel-Rehim, Molecularly imprinted polymer-sol-gel tablet toward micro-solid phase extraction: I. Determination of methadone in human plasma utilizing liquid chromatography-tandem mass spectrometry, Anal. Chim. Acta 936 (2016) 116-122.

[81] A. El-Beqqali, L.I. Andersson, A.D. Jeppsson, M. Abdel-Rehim, Molecularly imprinted polymer-sol-gel tablet toward micro-solid phase extraction: II. Determination of amphetamine in human urine samples by liquid chromatography-tandem mass spectrometry, J. Chromatogr. B 1063 (2017) $130-135$

[82] H. Li, D. Li, Preparation of a pipette tip-based molecularly imprinted solidphase microextraction monolith by epitope approach and its application for determination of enkephalins in human cerebrospinal fluid, J. Pharmaceut. Biomed. Anal. 115 (2015) 330-338.

[83] A. Sorribes-Soriano, R. Arráez-González, F.A. Esteve-Turrillas, S. Armenta, J.M. Herrero-Martínez, Development of a molecularly imprinted monolithic polymer disk for agitation-extraction of ecgonine methyl ester from environmental water, Talanta 199 (2019) 388-395.

[84] M. Arabi, M. Ghaedi, A. Ostovan, Synthesis and application of in-situ molecularly imprinted silica monolithic in pipette-tip solid-phase microextraction for the separation and determination of gallic acid in orange juice samples, J. Chromatogr. B 1048 (2017) 102-110.

[85] T. Bouvarel, N. Delaunay, V. Pichon, Selective extraction of cocaine from biological samples with a miniaturized monolithic molecularly imprinted polymer and on-line analysis in nano-liquid chromatography, Anal. Chim. Acta 1096 (2020) 89-99.

[86] S. Asır, D. Sarı, A. Derazshamshir, F. Yılmaz, K. Sarkaya, A. Denizli, Dopamineimprinted monolithic column for capillary electrochromatography, Electrophoresis 38 (2017) 3003-3012. 
[87] F.F. Yang, Z.X. Li, Y.J. Xu, Y.P. Huang, Z.S. Liu, Enhanced molecular recognition for imprinted monolithic column containing polyhedral oligomeric silsesquioxanes by dendritic effect of mesoporous molecular sieve scaffolds, Anal. Bioanal. Chem. 410 (2018) 5183-5193.

[88] L. Chen, X. Huang, Preparation and application of a poly (ionic liquid)-based molecularly imprinted polymer for multiple monolithic fiber solid-phase microextraction of phenolic acids in fruit juice and beer samples, Analyst 142 (2017) 4039-4047.

[89] M. Liu, T.M. Tran, A.A. Abbas Elhaj, S. Bøen Torsetnes, O.N. Jensen, B. Sellergren, K. Irgum, Molecularly Imprinted porous monolithic materials from melamine-formaldehyde for selective trapping of phosphopeptides, Anal. Chem. 89 (2017) 9491-9501.

[90] M. Liu, S.B. Torsetnes, C. Wierzbicka, O.N. Jensen, B. Sellergren, K. Irgum, Selective Enrichment of phosphorylated peptides by monolithic polymers surface imprinted with bis-imidazolium moieties by UV-initiated cryopolymerization, Anal. Chem. 91 (2019) 10188-10196.

[91] A. Poma, H. Brahmbhatt, H.M. Pendergraff, J.K. Watts, N.W. Turner, Generation of novel hybrid aptamer-molecularly imprinted polymeric nanoparticles, Adv. Mater. 27 (2015) 750-758.

[92] H. Lyu, H. Sun, Y. Zhu, J. Wang, Z. Xie, J. Li, A double-recognized aptamermolecularly imprinted monolithic column for high-specificity recognition of ochratoxin A, Anal. Chim. Acta 1103 (2020) 97-105.

[93] J. Xu, J. Chi, C. Lin, X. Lin, Z. Xie, Towards high-efficient online specific discrimination of zearalenone by using gold nanoparticles@aptamer-based affinity monolithic column, J. Chromatogr. A (2020) 461026.

[94] C. Wierzbicka, M. Liu, D. Bauer, K. Irgum, B. Sellergren, Cationic pTyr/pSer imprinted polymers based on a bis-imidazolium host monomer: phosphopeptide recognition in aqueous buffers demonstrated by $\mu$-liquid chromatography and monolithic columns, J. Mater. Chem. B 5 (2017) 953-960.

[95] M. Rutkowska, J. Płotka-Wasylka, C. Morrison, P.P. Wieczorek, J. Namieśnik, M. Marć, Application of molecularly imprinted polymers in analytical chiral separations and analysis, TrAC, Trends Anal. Chem. 102 (2018) 91-102.

[96] L. Ye, P.A.G. Cormack, K. Mosbach, Molecularly imprinted monodisperse microspheres for competitive radioassay, Anal. Commun. 36 (1999) 35-38.

[97] S. Beyazit, B. Tse Sum Bui, K. Haupt, C. Gonzato, Molecularly imprinted polymer nanomaterials and nanocomposites by controlled/living radical polymerization, Prog. Polym. Sci. 62 (2016) 1-21.

[98] J. Wackerlig, R. Schirhagl, Applications of molecularly imprinted polymer nanoparticles and their advances toward industrial use: a Review, Anal. Chem. 88 (2016) 250-261.

[99] H. Zhang, Molecularly imprinted nanoparticles for biomedical applications, Adv. Mater. 32 (2020) 1806328.

[100] M. Arabi, M. Ghaedi, A. Ostovan, J. Tashkhourian, H. Asadallahzadeh, Synthesis and application of molecularly imprinted nanoparticles combined ultrasonic assisted for highly selective solid phase extraction trace amount of celecoxib from human plasma samples using design expert (DXB) software, Ultrason. Sonochem. 33 (2016) 67-76.

[101] S. Farzaneh, E. Asadi, M. Abdouss, A. Barghi-Lish, S. Azodi-Deilami, H.A. Khonakdar, M. Gharghabi, Molecularly imprinted polymer nanoparticles for olanzapine recognition: application for solid phase extraction and sustained release, RSC Adv. 5 (2015) 9154-9166.

[102] L. Cenci, M. Bertolla, A. Anesi, E. Ambrosi, G. Guella, A.M. Bossi, Micro- versus nano-sized molecularly imprinted polymers in MALDI-TOF mass spectrometry analysis of peptides, Anal. Bioanal. Chem. 409 (2017) 6253-6261.

[103] M. Arabi, M. Ghaedi, A. Ostovan, Development of dummy molecularly imprinted based on functionalized silica nanoparticles for determination of acrylamide in processed food by matrix solid phase dispersion, Food Chem. 210 (2016) 78-84.

[104] Z. Chen, S.L. Hanna, L.R. Redfern, D. Alezi, T. Islamoglu, O.K. Farha, Reticular chemistry in the rational synthesis of functional zirconium cluster-based MOFs, Coord. Chem. Rev. 386 (2019) 32-49.

[105] L. Liu, Z. Qiao, X. Cui, C. Pang, H. Liang, P. Xie, X. Luo, Z. Huang, Y. Zhang, Z. Zhao, Amino acid imprinted UiO-66s for highly recognized adsorption of small angiotensin-converting-enzyme-inhibitory peptides, ACS Appl. Mater. Interfaces 11 (2019) 23039-23049.

[106] G. Wulff, W. Vesper, Preparation of chromatographic sorbents with chiral cavities for racemic resolution, J. Chromatogr. A 167 (1978) 171-186.

[107] W. Liu, C. Holdsworth, L. Ye, Synthesis of molecularly imprinted polymers using a functionalized initiator for chiral-selective recognition of propranolol, Chirality 32 (2020) 370-377.

[108] B.J. Jia, X. He, P.L. Cui, J.X. Liu, J.P. Wang, Detection of chloramphenicol in meat with a chemiluminescence resonance energy transfer platform based on molecularly imprinted graphene, Anal. Chim. Acta 1063 (2019) 136-143.

[109] Q. Zhao, H. Zhang, H. Zhao, J. Liu, J. Liu, Z. Chen, B. Li, X. Liao, J.M. Regenstein, J. Wang, X. Yang, Strategy of fusion covalent organic frameworks and molecularly imprinted polymers: a surprising effect in recognition and loading of cyanidin-3-O-glucoside, ACS Appl. Mater. Interfaces 12 (2020) $8751-8760$.

[110] J. Sánchez-González, M.J. Tabernero, A.M. Bermejo, P. Bermejo-Barrera, A. Moreda-Piñeiro, Development of magnetic molecularly imprinted polymers for solid phase extraction of cocaine and metabolites in urine before high performance liquid chromatography-tandem mass spectrometry, Talanta 147 (2016) 641-649.
[111] J. Luo, Y. Gao, K. Tan, W. Wei, X. Liu, Preparation of a magnetic molecularly imprinted graphene composite highly adsorbent for 4-nitrophenol in aqueous medium, ACS Sustain. Chem. Eng. 4 (2016) 3316-3326.

[112] M. Díaz-Álvarez, E. Turiel, A. Martín-Esteban, Molecularly imprinted polymer monolith containing magnetic nanoparticles for the stir-bar sorptive extraction of thiabendazole and carbendazim from orange samples, Anal. Chim. Acta 1045 (2019) 117-122.

[113] L. Huang, H. Zhai, G. Liang, Z. Su, K. Yuan, G. Lu, Y. Pan, Chip-based dualmolecularly imprinted monolithic capillary array columns coated Ag/GO for selective extraction and simultaneous determination of bisphenol A and nonyl phenol in fish samples, J. Chromatogr. A 1474 (2016) 14-22.

[114] Z. Iskierko, P.S. Sharma, D. Prochowicz, K. Fronc, F. D'Souza, D. Toczydłowska, F. Stefaniak, K. Noworyta, Molecularly imprinted polymer (MIP) film with improved surface area developed by using metal-organic framework (MOF) for sensitive lipocalin (NGAL) determination, ACS Appl. Mater. Interfaces 8 (2016) 19860-19865.

[115] Á. Santana-Mayor, B. Socas-Rodríguez, M.d.M. Afonso, J.A. Palenzuela-López, M.Á. Rodríguez-Delgado, Reduced graphene oxide-coated magnetic-nanoparticles as sorbent for the determination of phthalates in environmental samples by micro-dispersive solid-phase extraction followed by ultra-highperformance liquid chromatography tandem mass spectrometry, J. Chromatogr. A 1565 (2018) 36-47.

[116] G. Liang, H. Zhai, L. Huang, X. Tan, Q. Zhou, X. Yu, H. Lin, Synthesis of carbon quantum dots-doped dummy molecularly imprinted polymer monolithic column for selective enrichment and analysis of aflatoxin B1 in peanut, J. Pharmaceut. Biomed. Anal. 149 (2018) 258-264.

[117] H. Zhai, L. Huang, Z. Chen, Z. Su, K. Yuan, G. Liang, Y. Pan, Chip-based molecularly imprinted monolithic capillary array columns coated GO/SiO2 for selective extraction and sensitive determination of rhodamine B in chili powder, Food Chem. 214 (2017) 664-669.

[118] M. Arabi, M. Ghaedi, A. Ostovan, Development of a lower toxic approach based on green synthesis of water-compatible molecularly imprinted nanoparticles for the extraction of hydrochlorothiazide from human urine, ACS Sustain. Chem. Eng. 5 (2017) 3775-3785.

[119] O. Ramstroem, L.I. Andersson, K. Mosbach, Recognition sites incorporating both pyridinyl and carboxy functionalities prepared by molecular imprinting, J. Org. Chem. 58 (1993) 7562-7564.

[120] C. Sulitzky, B. Rückert, A.J. Hall, F. Lanza, K. Unger, B. Sellergren, Grafting of molecularly imprinted polymer films on silica supports containing surfacebound free radical initiators, Macromolecules 35 (2002), 3314-3314.

[121] G. Ramakers, G. Wackers, V. Trouillet, A. Welle, P. Wagner, T. Junkers, LaserGrafted molecularly imprinted polymers for the detection of histamine from organocatalyzed atom transfer radical polymerization, Macromolecules 52 (2019) 2304-2313.

[122] Z. Adali-Kaya, B. Tse Sum Bui, A. Falcimaigne-Cordin, K. Haupt, Molecularly imprinted polymer nanomaterials and nanocomposites: atom-transfer radical polymerization with acidic monomers, Angew. Chem. Int. Ed. 54 (2015) 5192-5195.

[123] S. Des Azevedo, D. Lakshmi, I. Chianella, M.J. Whitcombe, K. Karim, P.K. Ivanova-Mitseva, S. Subrahmanyam, S.A. Piletsky, Molecularly imprinted polymer-hybrid electrochemical sensor for the detection of $\beta$-estradiol, Ind. Eng. Chem. Res. 52 (2013) 13917-13923.

[124] A. Bhattacharya, B.N. Misra, Grafting: a versatile means to modify polymers: techniques, factors and applications, Prog. Polym. Sci. 29 (2004) 767-814.

[125] A.R. Bagheri, M. Arabi, M. Ghaedi, A. Ostovan, X.Y. Wang, J.H. Li, L.X. Chen, Dummy molecularly imprinted polymers based on a green synthesis strategy for magnetic solid-phase extraction of acrylamide in food samples, Talanta 195 (2019) 390-400.

[126] H. Li, R. Long, C. Tong, T. Li, Y. Liu, S. Shi, Shell thickness controlled hydrophilic magnetic molecularly imprinted resins for high-efficient extraction of benzoic acids in aqueous samples, Talanta 194 (2019) 969-976.

[127] Y.F. Wang, Y.G. Wang, X.K. Ouyang, L.Y. Yang, Surface-imprinted magnetic carboxylated cellulose nanocrystals for the highly selective extraction of six fluoroquinolones from egg samples, ACS Appl. Mater. Interfaces 9 (2017) 1759-1769.

[128] H. Liu, L. Mu, X. Chen, J. Wang, S. Wang, B. Sun, Core-shell metal-organic frameworks/molecularly imprinted nanoparticles as absorbents for the detection of pyrraline in milk and milk powder, J. Agric. Food Chem. 65 (2017) 986-992.

[129] N.M. Bergmann, N.A. Peppas, Molecularly imprinted polymers with specific recognition for macromolecules and proteins, Prog. Polym. Sci. 33 (2008) 271-288.

[130] J. Pan, W. Chen, Y. Ma, G. Pan, Molecularly imprinted polymers as receptor mimics for selective cell recognition, Chem. Soc. Rev. 47 (2018) 5574-5587.

[131] C. Boitard, A. Bée, C. Ménager, N. Griffete, Magnetic protein imprinted polymers: a review, J. Mater. Chem. B 6 (2018) 1563-1580.

[132] Z. Bie, Y. Chen, J. Ye, S. Wang, Z. Liu, Boronate-affinity glycan-oriented surface imprinting: a new strategy to mimic lectins for the recognition of an intact glycoprotein and its characteristic fragments, Angew. Chem. Int. Ed. 54 (2015) 10211-10215.

[133] K. Yang, S. Li, L. Liu, Y. Chen, W. Zhou, J. Pei, Z. Liang, L. Zhang, Y. Zhang, Epitope imprinting technology: progress, applications, and perspectives toward artificial antibodies, Adv. Mater. 31 (2019) 1902048. 
[134] C.J. Tan, S. Wangrangsimakul, R. Bai, Y.W. Tong, Defining the interactions between proteins and surfactants for nanoparticle surface imprinting through miniemulsion polymerization, Chem. Mater. 20 (2008) 118-127.

[135] K. Ren, N. Banaei, R.N. Zare, Sorting inactivated cells using cell-imprinted polymer thin films, ACS Nano 7 (2013) 6031-6036.

[136] M. Dąbrowski, A. Zimińska, J. Kalecki, M. Cieplak, W. Lisowski, R. Maksym, S. Shao, F. D'Souza, A. Kuhn, P.S. Sharma, Facile fabrication of surfaceimprinted macroporous films for chemosensing of human chorionic gonadotropin hormone, ACS Appl. Mater. Interfaces 11 (2019) 9265-9276.

[137] C. Rossetti, A. Abdel Qader, T.G. Halvorsen, B. Sellergren, L. Reubsaet, Antibody-free biomarker determination: exploring molecularly imprinted polymers for pro-gastrin releasing peptide, Anal. Chem. 86 (2014) 12291-12298.

[138] X. Li, J. Zhou, L. Tian, W. Li, Z. Ali, N. Ali, B. Zhang, H. Zhang, O Zhang, Effect of crosslinking degree and thickness of thermosensitive imprinted layers on recognition and elution efficiency of protein imprinted magnetic microspheres, Sensor. Actuator. B Chem. 225 (2016) 436-445.

[139] S.F. Xu, L.X. Chen, J.H. Li, W. Qin, J.P. Ma, Preparation of hollow porous molecularly imprinted polymers and their applications to solid-phase extraction of triazines in soil samples, J. Mater. Chem. 21 (2011) $12047-12053$

[140] Z. Zhang, S.F. Xu, J.H. Li, H. Xiong, H.L. Peng, L.X. Chen, Selective solid-phase extraction of sudan I in chilli sauce by single-hole hollow molecularly imprinted polymers, J. Agric. Food Chem. 60 (2012) 180-187.

[141] H. Gholami, M. Ghaedi, A. Ostovan, M. Arabi, A.R. Bagheri, Preparation of hollow porous molecularly imprinted and aluminum(III) doped silica nanospheres for extraction of the drugs valsartan and losartan prior to their quantitation by HPLC, Microchim. Acta 186 (2019) 702.

[142] Y. Hu, Q. Xia, W. Huang, X. Hou, M. Tian, Boronate-modified hollow molecularly imprinted polymers for selective enrichment of glycosides, Microchim. Acta 185 (2017) 46.

[143] X.Y. Wang, Q. Kang, D.Z. Shen, Z. Zhang, J.H. Li, L.X. Chen, Novel monodisperse molecularly imprinted shell for estradiol based on surface imprinted hollow vinyl-SiO 2 particles, Talanta 124 (2014) 7-13.

[144] S. Ji, T. Li, W. Yang, C. Shu, D. Li, Y. Wang, L. Ding, A hollow porous molecularly imprinted polymer as a sorbent for the extraction of 7 macrolide antibiotics prior to their determination by HPLC-MS/MS, Microchim. Acta 185 (2018) 203.

[145] L.N. Gómez-Arribas, J.L. Urraca, E. Benito-Peña, M.C. Moreno-Bondi, Tagspecific affinity purification of recombinant proteins by using molecularly imprinted polymers, Anal. Chem. 91 (2019) 4100-4106.

[146] H. Li, X. Hu, Y. Zhang, S. Shi, X. Jiang, X. Chen, High-capacity magnetic hollow porous molecularly imprinted polymers for specific extraction of protocatechuic acid, J. Chromatogr. A 1404 (2015) 21-27.

[147] H. Lu, S. Xu, Hollow mesoporous structured molecularly imprinted polymers for highly sensitive and selective detection of estrogens from food samples, J. Chromatogr. A 1501 (2017) 10-17.

[148] D. Fan, H. Li, S. Shi, X. Chen, Hollow molecular imprinted polymers towards rapid, effective and selective extraction of caffeic acid from fruits, J. Chromatogr. A 1470 (2016) 27-32.

[149] A. Wang, H. Lu, S. Xu, Preparation of magnetic hollow molecularly imprinted polymers for detection of triazines in food samples, J. Agric. Food Chem. 64 (2016) 5110-5116.

[150] X. Hou, B. Guo, Y. Tong, M. Tian, Using self-polymerization synthesis of boronate-affinity hollow stannic oxide based fragment template molecularly imprinted polymers for the selective recognition of polyphenols, J. Chromatogr. A 1612 (2020) 460631.

[151] A. Ostovan, M. Ghaedi, M. Arabi, A. Asfaram, Hollow porous molecularly imprinted polymer for highly selective clean-up followed by influentia preconcentration of ultra-trace glibenclamide from bio-fluid, J. Chromatogr. A 1520 (2017) 65-74.

[152] L.I. Andersson, A. Paprica, T. Arvidsson, A highly selective solid phase extraction sorbent for pre-concentration of sameridine made by molecular imprinting, Chromatographia 46 (1997) 57-62.

[153] M. Arabi, A. Ostovan, M. Ghaedi, M.K. Purkait, Novel strategy for synthesis of magnetic dummy molecularly imprinted nanoparticles based on functionalized silica as an efficient sorbent for the determination of acrylamide in potato chips: optimization by experimental design methodology, Talanta 154 (2016) 526-532.

[154] X. Wu, Y. Li, X. Zhu, C. He, O. Wang, S, Liu, Dummy molecularly imprinted magnetic nanoparticles for dispersive solid-phase extraction and determination of bisphenol A in water samples and orange juice, Talanta 162 (2017) 57-64.

[155] L. Tan, R. He, K. Chen, R. Peng, C. Huang, R. Yang, Y. Tang, Ultra-high performance liquid chromatography combined with mass spectrometry for determination of aflatoxins using dummy molecularly imprinted polymers deposited on silica-coated magnetic nanoparticles, Microchim. Acta 183 (2016) 1469-1477.

[156] F. Zhu, J. Wang, L. Zhu, L. Tan, G. Feng, S. Liu, Y. Dai, H. Wang, Preparation of molecularly imprinted polymers using theanine as dummy template and its application as SPE sorbent for the determination of eighteen amino acids in tobacco, Talanta 150 (2016) 388-398.

[157] L. Guo, X. Ma, X. Xie, R. Huang, M. Zhang, J. Li, G. Zeng, Y. Fan, Preparation of dual-dummy-template molecularly imprinted polymers coated magnetic graphene oxide for separation and enrichment of phthalate esters in water, Chem. Eng. J. 361 (2019) 245-255.
[158] M. Marć, T. Kupka, P.P. Wieczorek, J. Namieśnik, Computational modeling of molecularly imprinted polymers as a green approach to the development of novel analytical sorbents, TrAC, Trends Anal. Chem. 98 (2018) 64-78.

[159] X.Q. Cai, J.H. Li, Z. Zhang, F.F. Yang, R.C. Dong, L.X. Chen, Novel $\mathrm{Pb}^{2+}$ ion imprinted polymers based on ionic interaction via synergy of dual functional monomers for selective solid-phase extraction of $\mathrm{Pb}^{2+}$ in water samples, ACS Appl. Mater. Interfaces 6 (2014) 305-313.

[160] L. Ye, Molecularly imprinted polymers with multi-functionality, Anal. Bioanal. Chem. 408 (2016) 1727-1733.

[161] T. Cowen, K. Karim, S. Piletsky, Computational approaches in the design of synthetic receptors - a review, Anal. Chim. Acta 936 (2016) 62-74

[162] Z. Mazouz, M. Mokni, N. Fourati, C. Zerrouki, F. Barbault, M. Seydou, R. Kalfat, N. Yaakoubi, A. Omezzine, A. Bouslema, A. Othmane, Computational approach and electrochemical measurements for protein detection with MIP-based sensor, Biosens. Bioelectron. 151 (2020) 111978.

[163] X. Yu, H. Zeng, J. Wan, X. Cao, Computational design of a molecularly imprinted polymer compatible with an aqueous environment for solid phase extraction of chenodeoxycholic acid, J. Chromatogr. A 1609 (2020) 460490.

[164] M.V. Sullivan, S.R. Dennison, G. Archontis, S.M. Reddy, J.M. Hayes, Toward rational design of selective molecularly imprinted polymers (MIPs) for proteins: computational and experimental studies of acrylamide based polymers for myoglobin, J. Phys. Chem. B 123 (2019) 5432-5443.

[165] R.J. Umpleby, S.C. Baxter, M. Bode, J.K. Berch, R.N. Shah, K.D. Shimizu, Application of the Freundlich adsorption isotherm in the characterization of molecularly imprinted polymers, Anal. Chim. Acta 435 (2001) 35-42.

[166] R.J. Umpleby, S.C. Baxter, A.M. Rampey, G.T. Rushton, Y. Chen, K.D. Shimizu, Characterization of the heterogeneous binding site affinity distributions in molecularly imprinted polymers, J. Chromatogr. B 804 (2004) 141-149.

[167] A.M. Rampey, R.J. Umpleby, G.T. Rushton, J.C. Iseman, R.N. Shah, K.D. Shimizu, Characterization of the imprint effect and the influence of imprinting conditions on affinity, capacity, and heterogeneity in molecularly imprinted polymers using the freundlich isotherm-affinity distribution analysis, Anal. Chem. 76 (2004) 1123-1133.

[168] R. Xing, Y. Ma, Y. Wang, Y. Wen, Z. Liu, Specific recognition of proteins and peptides via controllable oriented surface imprinting of boronate affinityanchored epitopes, Chem. Sci. 10 (2019) 1831-1835.

[169] R. Xing, S. Wang, Z. Bie, H. He, Z. Liu, Preparation of molecularly imprinted polymers specific to glycoproteins, glycans and monosaccharides via boronate affinity controllable-oriented surface imprinting, Nat. Protoc. 12 (2017) 964-987.

[170] D. Li, Y. Chen, Z. Liu, Boronate affinity materials for separation and molecular recognition: structure, properties and applications, Chem. Soc. Rev. 44 (2015) 8097-8123.

[171] J. Wang, J. Dai, Y. Xu, X. Dai, Y. Zhang, W. Shi, B. Sellergren, G. Pan, Molecularly imprinted fluorescent test strip for direct, rapid, and visual dopamine detection in tiny amount of biofluid, Small 15 (2019) 1803913.

[172] C.L. Kao, Y.F. Chen, P.C. Huang, C.Y. Hsu, C.H. Kuei, A facile one-pot synthesis of L-DOPA imprinted silica nanospheres for chiral separation and in vitro controlled release, RSC Adv. 5 (2015) 15511-15514.

[173] F. Duan, C. Chen, X. Zhao, Y. Yang, X. Liu, Y. Oin, Water-compatible surface molecularly imprinted polymers with synergy of bi-functional monomers for enhanced selective adsorption of bisphenol A from aqueous solution, Environ. Sci. 3 (2016) 213-222.

[174] Y. Yuan, Y. Liu, W. Teng, J. Tan, Y. Liang, Y. Tang, Preparation of core-shell magnetic molecular imprinted polymer with binary monomer for the fast and selective extraction of bisphenol A from milk, J. Chromatogr. A 1462 (2016) 2-7.

[175] M. Arabi, M. Ghaedi, A. Ostovan, S. Wang, Synthesis of lab-in-a-pipette-tip extraction using hydrophilic nano-sized dummy molecularly imprinted polymer for purification and analysis of prednisolone, J. Colloid Interface Sci. 480 (2016) 232-239.

[176] J.Q. Fu, X.Y. Wang, J.H. Li, Y.J. Ding, L.X. Chen, Synthesis of multi-ion imprinted polymers based on dithizone chelation for simultaneous removal of $\mathrm{Hg}^{2+}, \mathrm{Cd}^{2+}, \mathrm{Ni}^{2+}$ and $\mathrm{Cu}^{2+}$ from aqueous solutions, RSC Adv. 6 (2016) 44087-44095.

[177] W.H. Lu, J.Y. Liu, J.H. Li, X.Y. Wang, M. Lv, R. Cui, L.X. Chen, Dual-template molecularly imprinted polymers for dispersive solid-phase extraction of fluoroquinolones in water samples coupled with high performance liquid chromatography, Analyst 144 (2019) 1292-1302.

[178] X.L. Song, J.H. Li, S.F. Xu, R.J. Ying, J.P. Ma, C.Y. Liao, D.Y. Liu, J.B. Yu, L.X. Chen, Determination of 16 polycyclic aromatic hydrocarbons in seawater using molecularly imprinted solid-phase extraction coupled with gas chromatography-mass spectrometry, Talanta 99 (2012) 75-82.

[179] W.H. Lu, X.Y. Wang, X.Q. Wu, D.Y. Liu, J.H. Li, L.X. Chen, X.S. Zhang, Multitemplate imprinted polymers for simultaneous selective solid-phase extraction of six phenolic compounds in water samples followed by determination using capillary electrophoresis, J. Chromatogr. A 1483 (2017) 30-39.

[180] C. Sun, J. Wang, J. Huang, D. Yao, C.Z. Wang, L. Zhang, S. Hou, L. Chen, C.S. Yuan, The multi-template molecularly imprinted polymer based on SBA15 for selective separation and determination of panax notoginseng saponins simultaneously in biological samples, Polymers 9 (2017) 653.

[181] A. Ostovan, M. Ghaedi, M. Arabi, Q. Yang, J.H. Li, L.X. Chen, Hydrophilic multitemplate molecularly imprinted biopolymers based on a green 
synthesis strategy for determination of B-family vitamins, ACS Appl. Mater. Interfaces 10 (2018) 4140-4150.

[182] M.J. Whitcombe, I. Chianella, L. Larcombe, S.A. Piletsky, J. Noble, R. Porter, A. Horgan, The rational development of molecularly imprinted polymerbased sensors for protein detection, Chem. Soc. Rev. 40 (2011) 1547-1571.

[183] H. Zhang, Water-compatible molecularly imprinted polymers: promising synthetic substitutes for biological receptors, Polymer 55 (2014) 699-714.

[184] M. Arabi, A. Ostovan, A.R. Bagheri, X. Guo, J. Li, J. Ma, L. Chen, Hydrophilic molecularly imprinted nanospheres for the extraction of rhodamine B followed by HPLC analysis: a green approach and hazardous waste elimination, Talanta 215 (2020) 120933.

[185] G. Vlatakis, L.I. Andersson, R. Müller, K. Mosbach, Drug assay using antibody mimics made by molecular imprinting, Nature 361 (1993) 645-647.

[186] H. Gholami, M. Arabi, M. Ghaedi, A. Ostovan, A.R. Bagheri, Column packing elimination in matrix solid phase dispersion by using water compatible magnetic molecularly imprinted polymer for recognition of melamine from milk samples, J. Chromatogr. A 1594 (2019) 13-22.

[187] W. Ji, R. Sun, W. Duan, X. Wang, T. Wang, Y. Mu, L. Guo, Selective solid phase extraction of chloroacetamide herbicides from environmental water samples by amphiphilic magnetic molecularly imprinted polymers, Talanta 170 (2017) 111-118.

[188] W. Ji, T. Wang, W. Liu, F. Liu, L. Guo, Y. Geng, X. Wang, Water-compatible micron-sized monodisperse molecularly imprinted beads for selective extraction of five iridoid glycosides from Cornus officinalis fructus, J. Chromatogr. A 1504 (2017) 1-8.

[189] W.H. Ji, Y.S. Guo, X. Wang, D.S. Guo, A water-compatible magnetic molecularly imprinted polymer for the selective extraction of risperidone and 9hydroxyrisperidone from human urine, Talanta 181 (2018) 392-400.

[190] H. Gholami, M. Ghaedi, M. Arabi, A. Ostovan, A.R. Bagheri, H. Mohamedian, Application of molecularly imprinted biomembrane for advancement of matrix solid-phase dispersion for clean enrichment of parabens from powder sunscreen samples: optimization of chromatographic conditions and green approach, ACS Omega 4 (2019) 3839-3849.

[191] E. Benito-Peña, J.L. Urraca, B. Sellergren, M.C. Moreno-Bondi, Solid-phase extraction of fluoroquinolones from aqueous samples using a watercompatible stochiometrically imprinted polymer, J. Chromatogr. A 1208 (2008) 62-70.

[192] P. Manesiotis, C. Borrelli, C.S.A. Aureliano, C. Svensson, B. Sellergren, Watercompatible imprinted polymers for selective depletion of riboflavine from beverages, J. Mater. Chem. 19 (2009) 6185-6193.

[193] G. Pan, Y. Zhang, Y. Ma, C. Li, H. Zhang, Efficient one-pot synthesis of watercompatible molecularly imprinted polymer microspheres by facile RAFT precipitation polymerization, Angew. Chem. Int. Ed. 50 (2011) 11731-11734.

[194] Y. Ma, G. Pan, Y. Zhang, X. Guo, H. Zhang, Narrowly dispersed hydrophilic molecularly imprinted polymer nanoparticles for efficient molecular recognition in real aqueous samples including river water, milk, and bovine serum, Angew. Chem. Int. Ed. 52 (2013) 1511-1514.

[195] X. Zhao, J. Wang, J. Wang, S. Wang, Development of water-compatible molecularly imprinted solid-phase extraction coupled with high performance liquid chromatography-tandem mass spectrometry for the detection of six sulfonamides in animal-derived foods, J. Chromatogr. A 1574 (2018) $9-17$.

[196] X.Q. Wu, X.Y. Wang, W.H. Lu, X.Y. Wang, J.H. Li, H.Y. You, H. Xiong, L.X. Chen, Water-compatible temperature and magnetic dual-responsive molecularly imprinted polymers for recognition and extraction of bisphenol A, J. Chromatogr. A 1435 (2016) 30-38.

[197] Y. Ma, H. Li, S. He, H. Zhang, H. Zhang, Efficient one-pot synthesis of watercompatible and photoresponsive molecularly imprinted polymer nanoparticles by facile RAFT precipitation polymerization, J. Polym. Sci., Polym. Chem. Ed. 52 (2014) 1941-1952.

[198] Y. Ma, J. Gao, C. Zheng, H. Zhang, Well-defined biological sample-compatible molecularly imprinted polymer microspheres by combining RAFT polymerization and thiol-epoxy coupling chemistry, J. Mater. Chem. B 7 (2019) 2474-2483.

[199] M. Zhao, C. Zhang, Y. Zhang, X. Guo, H. Yan, H. Zhang, Efficient synthesis of narrowly dispersed hydrophilic and magnetic molecularly imprinted polymer microspheres with excellent molecular recognition ability in a real biological sample, Chem. Commun. 50 (2014) 2208-2210.

[200] X. Lu, C. Zheng, H. Zhang, Improvement of surface hydrophilicity and biological sample-compatibility of molecularly imprinted polymer microspheres by facile surface modification with $\alpha$-cyclodextrin, Eur. Polym. J. 115 (2019) 12-21.

[201] M. Wang, X. Chang, X. Wu, H. Yan, F. Qiao, Water-compatible dummy molecularly imprinted resin prepared in aqueous solution for green miniaturized solid-phase extraction of plant growth regulators, J. Chromatogr. A 1458 (2016) 9-17.

[202] M. Wang, S. Liang, L. Bai, F. Qiao, H. Yan, Green protocol for the preparation of hydrophilic molecularly imprinted resin in water for the efficient selective extraction and determination of plant hormones from bean sprouts, Anal. Chim. Acta 1064 (2019) 47-55.

[203] Y. Yuan, C. Yang, T. Lv, F. Qiao, Y. Zhou, H. Yan, Green synthesis of hydrophilic protein-imprinted resin with specific recognition of bovine serum albumin in aqueous matrix, Anal. Chim. Acta 1033 (2018) 213-220.
[204] G.d.O.I. Moraes, L.M.R. da Silva, Á.J. dos Santos-Neto, F.H. Florenzano, E.C. Figueiredo, A new restricted access molecularly imprinted polymer capped with albumin for direct extraction of drugs from biological matrices: the case of chlorpromazine in human plasma, Anal. Bioanal. Chem. 405 (2013) 7687-7696.

[205] T. Liang, L. Chen, Y. Ma, Mesoporous structured molecularly imprinted polymer with restricted access function for highly selective extraction of chlorpyrifos from soil, J. Chromatogr. A 1609 (2020) 460453.

[206] J. Haginaka, H. Takehira, K. Hosoya, N. Tanaka, Uniform-sized molecularly imprinted polymer for (S)-naproxen selectively modified with hydrophilic external layer, J. Chromatogr. A 849 (1999) 331-339.

[207] M. Arabi, M. Ghaedi, A. Ostovan, Water compatible molecularly imprinted nanoparticles as a restricted access material for extraction of hippuric acid, a biological indicator of toluene exposure, from human urine, Microchim. Acta 184 (2017) 879-887.

[208] H.G. Zuo, J.X. Zhu, C.R. Zhan, G.Y. Tang, P. Guo, Y.L. Wei, H.L. Zeng, H. Yang A method developed for determination of heptachlor and its metabolites from pork, Environ. Monit. Assess. 186 (2014) 2399-2412.

[209] H.G. Zuo, H. Yang, J.X. Zhu, Y. Ding, Preparation of a novel RAM-MIP for selective solid-phase extraction and gas chromatography determination of heptachlor, endosulfan and their metabolite residues in pork, Anal. Methods 9 (2017) 6009-6018.

[210] I.D. Souza, L.P. Melo, I.C. Jardim, J.C. Monteiro, A.M.S. Nakano, M.E.C. Queiroz, Selective molecularly imprinted polymer combined with restricted access material for in-tube SPME/UHPLC-MS/MS of parabens in breast milk samples, Anal. Chim. Acta 932 (2016) 49-59.

[211] R. Rodríguez-Gómez, I. Jiménez-Díaz, A. Zafra-Gómez, O. Ballesteros A. Navalón, A multiresidue method for the determination of selected endocrine disrupting chemicals in human breast milk based on a simple extraction procedure, Talanta 130 (2014) 561-570.

[212] J. He, L. Song, S. Chen, Y. Li, H. Wei, D. Zhao, K. Gu, S. Zhang, Novel restricted access materials combined to molecularly imprinted polymers for selective solid-phase extraction of organophosphorus pesticides from honey, Food Chem. 187 (2015) 331-337.

[213] B. Du, T. Qu, Z. Chen, X. Cao, S. Han, G. Shen, L. Wang, A novel restricted access material combined to molecularly imprinted polymers for selective solid-phase extraction and high performance liquid chromatography determination of 2-methoxyestradiol in plasma samples, Talanta 129 (2014) 465-472.

[214] B. Claude, C. Berho, S. Bayoudh, L. Amalric, E. Coisy, R. Nehmé, P. Morin, Preliminary recovery study of a commercial molecularly imprinted polymer for the extraction of glyphosate and AMPA in different environmental waters using MS, Environ. Sci. Pol. 24 (2017) 12293-12300.

[215] D. Mutavdžić Pavlović, K. Nikšić, S. Livazović, I. Brnardić, A. Anžlovar, Preparation and application of sulfaguanidine-imprinted polymer on solid-phase extraction of pharmaceuticals from water, Talanta 131 (2015) 99-107.

[216] A. Poma, A. Guerreiro, M.J. Whitcombe, E.V. Piletska, A.P.F. Turner, S.A. Piletsky, Solid-phase synthesis of molecularly imprinted polymer nanoparticles with a reusable Template-"Plastic antibodies", Adv. Funct. Mater. 23 (2013) 2821-2827.

[217] M. Berghaus, R. Mohammadi, B. Sellergren, Productive encounter: molecularly imprinted nanoparticles prepared using magnetic templates, Chem. Commun. 50 (2014) 8993-8996.

[218] S. Ambrosini, S. Beyazit, K. Haupt, B. Tse Sum Bui, Solid-phase synthesis of molecularly imprinted nanoparticles for protein recognition, Chem. Commun. 49 (2013) 6746-6748.

[219] A. Poma, A. Guerreiro, S. Caygill, E. Moczko, S. Piletsky, Automatic reactor for solid-phase synthesis of molecularly imprinted polymeric nanoparticles (MIP NPs) in water, RSC Adv. 4 (2014) 4203-4206.

[220] W. Liu, L. Qin, W. Shi, L. Chen, Y. Yang, X. Liu, B. Xu, Molecularly imprinted polymers on the surface of porous carbon microspheres for capturing dibenzothiophene, Microchim. Acta 183 (2016) 1153-1160.

[221] X. Tian, C. She, Z. Qi, X. Xu, Microchem. J. 146 (2019) 1126-1133.

[222] X. Li, Y. Dai, K.H. Row, Preparation of two-dimensional magnetic molecularly imprinted polymers based on boron nitride and a deep eutectic solvent for the selective recognition of flavonoids, Analyst 144 (2019) 1777-1788.

[223] R. Yang, Y. Liu, X. Yan, S. Liu, Simultaneous extraction and determination of phthalate esters in aqueous solution by yolk-shell magnetic mesoporous carbon-molecularly imprinted composites based on solid-phase extraction coupled with gas chromatography-mass spectrometry, Talanta 161 (2016) $114-121$.

[224] A.M. Chrzanowska, A. Poliwoda, P.P. Wieczorek, Surface molecularly imprinted silica for selective solid-phase extraction of biochanin A, daidzein and genistein from urine samples, J. Chromatogr. A 1392 (2015) 1-9.

[225] Y.-H. Ge, H. Shu, X.-Y. Xu, P.-Q. Guo, R.-L. Liu, Z.-M. Luo, C. Chang, Q. Fu, Combined magnetic porous molecularly imprinted polymers and deep eutectic solvents for efficient and selective extraction of aristolochic acid I and II from rat urine, Mater. Sci. Eng. C 97 (2019) 650-657.

[226] A. Ostovan, M. Ghaedi, M. Arabi, Fabrication of water-compatible superparamagnetic molecularly imprinted biopolymer for clean separation of baclofen from bio-fluid samples: a mild and green approach, Talanta 179 (2018) 760-768.

[227] A. Vicario, M. Solari, E. Felici, L. Aragón, F. Bertolino, M.R. Gomez, Molecular imprinting on surface of silica particles for the selective extraction of 
benzylparaben in flow system applied to cosmetics and water samples, Microchem. J. 142 (2018) 329-334.

[228] H. Wang, Y. Liu, S. Yao, P. Zhu, Selective recognization of dicyandiamide in bovine milk by mesoporous silica SBA-15 supported dicyandiamide imprinted polymer based on surface molecularly imprinting technique, Food Chem. 240 (2018) 1262-1267.

[229] X. Wang, P. Huang, X. Ma, X. Du, X. Lu, Magnetic mesoporous molecularly imprinted polymers based on surface precipitation polymerization for selective enrichment of triclosan and triclocarban, J. Chromatogr. A 1537 (2018) 35-42.

[230] Y.Z. Zhang, J.W. Zhang, C.Z. Wang, L.D. Zhou, Q.H. Zhang, C.S. Yuan, Polydopamine-coated magnetic molecularly imprinted polymers with fragment template for identification of Pulsatilla saponin metabolites in rat feces with UPLC-Q-TOF-MS, J. Agric. Food Chem. 66 (2018) 653-660.

[231] Z. Zhang, D. Niu, Y. Li, J. Shi, Magnetic, core-shell structured and surface molecularly imprinted polymers for the rapid and selective recognition of salicylic acid from aqueous solutions, Appl. Surf. Sci. 435 (2018) 178-186.

[232] X. Sun, M. Wang, J. Peng, L. Yang, X. Wang, F. Wang, X. Zhang, Q. Wu, R. Chen, J. Chen, Dummy molecularly imprinted solid phase extraction of climbazole from environmental water samples, Talanta 196 (2019) 47-53.

[233] M. Marć, A. Panuszko, J. Namieśnik, P.P. Wieczorek, Preparation and characterization of dummy-template molecularly imprinted polymers as potential sorbents for the recognition of selected polybrominated dipheny ethers, Anal. Chim. Acta 1030 (2018) 77-95.

[234] X. Sun, J. Peng, M. Wang, J. Wang, C. Tang, L. Yang, H. Lei, F. Li, X. Wang, J. Chen, Determination of nine bisphenols in sewage and sludge using dummy molecularly imprinted solid-phase extraction coupled with liquid chromatography tandem mass spectrometry, J. Chromatogr. A 1552 (2018) 10-16.

[235] P. Guo, X. Yuan, J. Zhang, B. Wang, X. Sun, X. Chen, L. Zhao, Dummy-surface molecularly imprinted polymers as a sorbent of micro-solid-phase extraction combined with dispersive liquid-liquid microextraction for determination of five 2-phenylpropionic acid NSAIDs in aquatic environmental samples, Anal. Bioanal. Chem. 410 (2018) 373-389.

[236] X. He, J. Chen, J. Wang, L. Tan, Multipoint recognition of domoic acid from seawater by dummy template molecularly imprinted solid-phase extraction coupled with high-performance liquid chromatography, J. Chromatogr. A 1500 (2017) 61-68.

[237] X. Hu, X. Wu, F. Yang, Q. Wang, C. He, S. Liu, Novel surface dummy molecularly imprinted silica as sorbent for solid-phase extraction of bisphenol A from water samples, Talanta 148 (2016) 29-36.

[238] Y. Liu, D. Wang, F. Du, W. Zheng, Z. Liu, Z. Xu, X. Hu, H. Liu, Dummy-template molecularly imprinted micro-solid-phase extraction coupled with highperformance liquid chromatography for bisphenol A determination in environmental water samples, Microchem. J. 145 (2019) 337-344.

[239] X. Sun, M. Wang, L. Yang, H. Wen, L. Wang, T. Li, C. Tang, J. Yang, Preparation and evaluation of dummy-template molecularly imprinted polymer as a potential sorbent for solid phase extraction of imidazole fungicides from river water, J. Chromatogr. A 1586 (2019) $1-8$

[240] J. Zhu, D. Chen, Y. Ai, X. Dang, J. Huang, H. Chen, A dummy molecularly imprinted monolith for selective solid-phase microextraction of vanillin and methyl vanillin prior to their determination by HPLC, Microchim. Acta 184 (2017) 1161-1167.

[241] J. Sánchez-González, S. García-Carballal, P. Cabarcos, M.J. Tabernero, P. Bermejo-Barrera, A. Moreda-Piñeiro, Determination of cocaine and its metabolites in plasma by porous membrane-protected molecularly imprinted polymer micro-solid-phase extraction and liquid chromatography - tandem mass spectrometry, J. Chromatogr. A 1451 (2016) 15-22.

[242] D.V.M. Sousa, F.V. Pereira, C.C. Nascentes, J.S. Moreira, V.H.M. Boratto, R.M. Orlando, Cellulose cone tip as a sorbent material for multiphase electrical field-assisted extraction of cocaine from saliva and determination by LC-MS/MS, Talanta 208 (2020) 120353.

[243] M.N.H. Rozaini, N.-f. Semail, B. Saad, S. Kamaruzaman, W.N. Abdullah, N.A. Rahim, M. Miskam, S.H. Loh, N. Yahaya, Molecularly imprinted silica gel incorporated with agarose polymer matrix as mixed matrix membrane for separation and preconcentration of sulfonamide antibiotics in water samples, Talanta 199 (2019) 522-531.

[244] Z. Chen, C. Yu, J. Xi, S. Tang, T. Bao, J. Zhang, A hybrid material prepared by controlled growth of a covalent organic framework on amino-modified MIL68 for pipette tip solid-phase extraction of sulfonamides prior to thei determination by HPLC, Microchim. Acta 186 (2019) 393.

[245] N. Ferreirós, G. Iriarte, R.M. Alonso, R.M. Jiménez, E. Ortíz, Separation and quantitation of several angiotensin II receptor antagonist drugs in human urine by a SPE-HPLC-DAD method, J. Separ. Sci. 31 (2008) 667-676.

[246] N. Harun, R.A. Anderson, P.A.G. Cormack, Analysis of ketamine and norketamine in hair samples using molecularly imprinted solid-phase extraction (MISPE) and liquid chromatography-tandem mass spectrometry (LC-MS/ MS), Anal. Bioanal. Chem. 396 (2010) 2449-2459.

[247] B. Hu, B. Zheng, D. Rickert, G.A. Gómez-Ríos, B. Bojko, J. Pawliszyn, Z.-P. Yao, Direct coupling of solid phase microextraction with electrospray ionization mass spectrometry: a Case study for detection of ketamine in urine, Anal. Chim. Acta 1075 (2019) 112-119.

[248] R.M. Toudeshki, S. Dadfarnia, A.M. Haji Shabani, Surface molecularly imprinted polymer on magnetic multi-walled carbon nanotubes for selective recognition and preconcentration of metformin in biological fluids prior to its sensitive chemiluminescence determination: central composite design optimization, Anal. Chim. Acta 1089 (2019) 78-89.
[249] B. Abbas Moussa, M.A. Mahrouse, M.G. Fawzy, A validated LC-MS/MS method for simultaneous determination of linagliptin and metformin in spiked human plasma coupled with solid phase extraction: application to a pharmacokinetic study in healthy volunteers, J. Pharmaceut. Biomed. Anal. 163 (2019) 153-161.

[250] Y. Huang, C. Li, H. Hu, Y. Wang, M. Shen, S. Nie, J. Chen, M. Zeng, M. Xie, Simultaneous determination of acrylamide and 5-hydroxymethylfurfural in heat-processed foods employing enhanced matrix removal-lipid as a new dispersive solid-phase extraction sorbent followed by liquid chromatography-tandem mass spectrometry, J. Agric. Food Chem. 67 (2019) 5017-5025.

[251] N. Nouri, H. Sereshti, Electrospun polymer composite nanofiber-based insyringe solid phase extraction in tandem with dispersive liquid-liquid microextraction coupled with HPLC-FD for determination of aflatoxins in soybean, Food Chem. 289 (2019) 33-39.

[252] Y. Liu, Y. Liu, Z. Liu, F. Du, G. Oin, G. Li, X. Hu, Z. Xu, Z. Cai, Supramolecularly imprinted polymeric solid phase microextraction coatings for synergetic recognition nitrophenols and bisphenol A, J. Hazard Mater. 368 (2019) 358-364.

[253] E. Marzi Khosrowshahi, A.A. Matin, A monolithic graphitic carbon nitride/polyethersulfone nanocomposite: an application of a mixed matrix membrane as a solid-phase microextraction fiber, Microchim. Acta 186 (2019) 679.

[254] L. Gao, L. Chen, X. Li, Magnetic molecularly imprinted polymers based on carbon nanotubes for extraction of carbamates, Microchim. Acta 182 (2015) $781-787$

[255] P. Salisaeng, P. Arnnok, N. Patdhanagul, R. Burakham, Vortex-assisted dispersive micro-solid phase extraction using CTAB-modified zeolite NaY sorbent coupled with HPLC for the determination of carbamate insecticides, J. Agric. Food Chem. 64 (2016) 2145-2152.

[256] S. Wang, Y. She, S. Hong, X. Du, M. Yan, Y. Wang, Y. Qi, M. Wang, W. Jiang, J. Wang, Dual-template imprinted polymers for class-selective solid-phase extraction of seventeen triazine herbicides and metabolites in agro-products, J. Hazard Mater. 367 (2019) 686-693.

[257] Z. Qin, Y. Jiang, H. Piao, J. Li, S. Tao, P. Ma, X. Wang, D. Song, Y. Sun, MIL101(Cr)/MWCNTs-functionalized melamine sponges for solid-phase extraction of triazines from corn samples, and their subsequent determination by HPLC-MS/MS, Talanta 211 (2020) 120676.

[258] A. Azizi, F. Shahhoseini, C.S. Bottaro, Magnetic molecularly imprinted polymers prepared by reversible addition fragmentation chain transfer polymerization for dispersive solid phase extraction of polycyclic aromatic hydrocarbons in water, J. Chromatogr. A 1610 (2020) 460534.

[259] S.M.S. Jillani, M. Sajid, K. Alhooshani, Evaluation of carbon foam as an adsorbent in stir-bar supported micro-solid-phase extraction coupled with gas chromatography-mass spectrometry for the determination of polyaromatic hydrocarbons in wastewater samples, Microchem. J. 144 (2019) 361-368.

[260] S.Y. Ma, X. Wang, H.L. Duan, J. Wang, H.Y. Zhan, Z.Q. Zhang, A nanoporous carbon derived from bimetallic organic-framework for magnetic solid-phase extraction of bisphenol analogs, Talanta 202 (2019) 479-485.

[261] X. Xie, L. Chen, X. Pan, S. Wang, Synthesis of magnetic molecularly imprinted polymers by reversible addition fragmentation chain transfer strategy and its application in the Sudan dyes residue analysis, J. Chromatogr. A 1405 (2015) 32-39.

[262] X. Ling, Z. Chen, Immobilization of zeolitic imidazolate frameworks with assist of electrodeposited zinc oxide layer and application in online solidphase microextraction of Sudan dyes, Talanta 192 (2019) 142-146.

[263] S. Tan, H. Yu, Y. He, M. Wang, G. Liu, S. Hong, F. Yan, Y. Wang, M. Wang, T. Li, J. Wang, A.M. Abd Ei-Aty, A. Hacımüftüoğlu, Y. She, A dummy molecularly imprinted solid-phase extraction coupled with liquid chromatographytandem mass spectrometry for selective determination of four pyridine carboxylic acid herbicides in milk, J. Chromatogr. B 1108 (2019) 65-72.

[264] M. Lashgari, C. Basheer, H. Kee Lee, Application of surfactant-templated ordered mesoporous material as sorbent in micro-solid phase extraction followed by liquid chromatography-triple quadrupole mass spectrometry for determination of perfluorinated carboxylic acids in aqueous media, Talanta 141 (2015) 200-206.

[265] F. Omidi, M. Behbahani, M. Khadem, F. Golbabaei, S.J. Shahtaheri, Application of ultrasonication for facilitating the extraction of hippuric acid and methyl hippuric acid in real samples using $\mathrm{Fe}_{3} \mathrm{O}_{4} @ \mathrm{SiO}_{2} @$ sodium dodecyl sulfate: experimental design methodology, Anal. Methods 10 (2018) 4588-4595.

[266] Y. Yin, L. Yan, Z. Zhang, J. Wang, Magnetic molecularly imprinted polydopamine nanolayer on multiwalled carbon nanotubes surface for protein capture, Talanta 144 (2015) 671-679.

[267] Y. Huang, Y. Wang, O. Pan, Y. Wang, X. Ding, K. Xu, N. Li, O. Wen, Magnetic graphene oxide modified with choline chloride-based deep eutectic solvent for the solid-phase extraction of protein, Anal. Chim. Acta 877 (2015) 90-99.

[268] Q. Zhan, H. Zhao, Y. Hong, C. Pu, Y. Liu, M. Lan, Preparation of a hydrophilic interaction liquid chromatography material by sequential electrostatic deposition of layers of polyethyleneimine and hyaluronic acid for enrichment of glycopeptides, Microchim. Acta 186 (2019) 600.

[269] X. Wei, Y. Wang, J. Chen, R. Ni, J. Meng, Z. Liu, F. Xu, Y. Zhou, Ionic liquids skeleton typed magnetic core-shell molecularly imprinted polymers for the specific recognition of lysozyme, Anal. Chim. Acta 1081 (2019) 81-92.

[270] H.L. Duan, Q.L. Niu, J. Wang, S.Y. Ma, J. Zhang, Z.Q. Zhang, High uptake carboxyl-functionalized porous $\beta$-cyclodextrin polymer for selective extraction of lysozyme from egg white, J. Chromatogr. A 1600 (2019) 80-86. 